\title{
Spectral and Quantum Dynamical Properties of the Weakly Coupled Fibonacci Hamiltonian
}

\author{
David Damanik $^{1, \star}$, Anton Gorodetski ${ }^{2, \star \star}$ \\ 1 Department of Mathematics, Rice University, Houston, TX 77005, USA. \\ E-mail: damanik@rice.edu \\ 2 Department of Mathematics, University of California, Irvine, CA 92697, USA. \\ E-mail: asgor@math.uci.edu
}

Received: 31 July 2010 / Accepted: 5 October 2010

Published online: 12 March 2011 - (C) The Author(s) 2011. This article is published with open access at Springerlink.com

\begin{abstract}
We consider the spectrum of the Fibonacci Hamiltonian for small values of the coupling constant. It is known that this set is a Cantor set of zero Lebesgue measure. Here we study the limit, as the value of the coupling constant approaches zero, of its thickness and its Hausdorff dimension. We prove that the thickness tends to infinity and, consequently, the Hausdorff dimension of the spectrum tends to one. We also show that at small coupling, all gaps allowed by the gap labeling theorem are open and the length of every gap tends to zero linearly. Moreover, for a sufficiently small coupling, the sum of the spectrum with itself is an interval. This last result provides a rigorous explanation of a phenomenon for the Fibonacci square lattice discovered numerically by Even-Dar Mandel and Lifshitz. Finally, we provide explicit upper and lower bounds for the solutions to the difference equation and use them to study the spectral measures and the transport exponents.
\end{abstract}

\section{Contents}

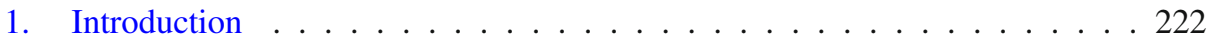

1.1 Background and motivation . . . . . . . . . . . . . . . . . 222

1.2 Statement of the main results . . . . . . . . . . . . . . . . 223

1.3 Overview of the paper . . . . . . . . . . . . . . . . . 228

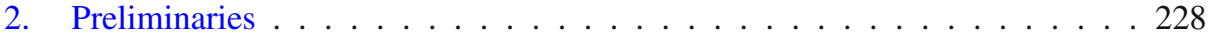

2.1 Description of the trace map and previous results . . . . . . . . . 228

2.2 Properties of the trace map for $V=0 \ldots \ldots . \ldots . \ldots 231$

3. The Spectrum as a Set . . . . . . . . . . . . . . . . . 234

3.1 Linear gap opening as the potential is turned on . . . . . . . . 235

3.2 Choice of a coordinate system in a neighborhood of a singular point . . 238

^ D. D. was supported in part by NSF grants DMS-0653720 and DMS-0800100.

$\star \star$ A. G. was supported in part by NSF grant DMS-0901627. 
3.3 Ordering of the gaps . . . . . . . . . . . . . . . . . . . 240

3.4 Distortion property: estimate of the gap sizes . . . . . . . . . . . . 241

3.5 Dynamics near singularities . . . . . . . . . . . . . . . . . . . 242

3.6 Distortion property: preliminary estimates _ . . . . . . . . . 250

3.7 Proof of the distortion property . . . . . . . . . . . . . . . . 253

4. The Integrated Density of States . . . . . . . . . . . . . . . . . 253

4.1 Definition and basic properties . . . . . . . . . . . . . 253

4.2 Complete gap labeling . . . . . . . . . . . . . . . . 254

4.3 More on the asymptotic gap lengths . . . . . . . . . . . . . 255

5. Spectral Measures and Transport Exponents _ . . . . . . . . . . . . 256

5.1 Solution estimates . . . . . . . . . . . . . . . . . . . 256

5.2 Spectral measures . . . . . . . . . . . . . . . . . . . . 264

5.3 Transport exponents . . . . . . . . . . . . . . . . . . . . 264

6. Consequences for Higher-Dimensional Models . . . . . . . . . . . . . 266

6.1 Lattice Schrödinger operators with separable potentials . . . . . . . . 266

6.2 A consequence of the Newhouse Gap Lemma . . . . . . . . . . . . 267

6.3 The square Fibonacci Hamiltonian . . . . . . . . . . . . . . . . 267

Appendix A. The Off-Diagonal Fibonacci Hamiltonian _ . . . . . . . . . . 268

A.1. Model and results . . . . . . . . . . . . . . . . . . 268

A.2. The trace map and its relation to the spectrum . . . . . . . . . 270

A.3. Singular continuous spectrum _ . . . . . . . . . . . . . . . 274

References . . . . . . . . . . . . . . . . . . . . . 275

\section{Introduction}

1.1. Background and motivation. It is always exciting to obtain a new connection between two different areas of mathematics. Here we prove several new results concerning the spectral properties of the discrete Schrödinger operator with Fibonacci potential, the so-called Fibonacci Hamiltonian, using methods from the modern theory of dynamical systems (uniformly hyperbolic and normally hyperbolic dynamics).

The Fibonacci Hamiltonian is a central model in the study of electronic properties of one-dimensional quasicrystals. It is given by the following bounded self-adjoint operator in $\ell^{2}(\mathbb{Z})$ :

$$
\left[H_{V, \omega} \psi\right](n)=\psi(n+1)+\psi(n-1)+V \chi_{[1-\alpha, 1)}(n \alpha+\omega \bmod 1) \psi(n),
$$

where $V>0, \alpha=\frac{\sqrt{5}-1}{2}$, and $\omega \in \mathbb{T}=\mathbb{R} / \mathbb{Z}$.

This operator family has been studied in many papers since the early 1980's and numerous fundamental results are known. Let us recall some of them and refer the reader to the survey articles [D00,D07a,S95] for additional information.

The spectrum is easily seen to be independent of $\omega$ and may therefore be denoted by $\Sigma_{V}$. That is, $\sigma\left(H_{V, \omega}\right)=\Sigma_{V}$ for every $\omega \in \mathbb{T}$. Indeed, this follows quickly from the minimality of the irrational rotation by $\alpha$ and strong operator convergence. It was shown by Sütôt that $\Sigma_{V}$ has zero Lebesgue measure for every $V>0$; see [S89]. Moreover, it is compact (since it is the spectrum of a bounded operator) and perfect (because the irrational rotation by $\alpha$ is ergodic). Thus, $\Sigma_{V}$ is a zero-measure Cantor set. This result was recently strengthened by Cantat [Can] who showed that the Hausdorff dimension of $\Sigma_{V}$ lies strictly between zero and one. 
Naturally, one is interested in fractal properties of $\Sigma_{V}$, such as its dimension, thickness, and denseness. While such a study is well-motivated from a purely mathematical perspective, we want to point out that there is significant additional interest in these quantities. In particular, it has recently been realized that the fractal dimension of the spectrum is intimately related with the long-time asymptotics of the solution to the associated time-dependent Schrödinger equation, that is, $i \partial_{t} \phi=H_{V, \omega} \phi$; see [DEGT].

Fractal properties of $\Sigma_{V}$ are by now well understood for large values of $V$. Work of Casdagli [Cas] and Sütő [S87] shows that for $V \geq 16, \Sigma_{V}$ is a dynamically defined Cantor set. It follows from this result that the Hausdorff dimension and the upper and lower box counting dimension of $\Sigma_{V}$ all coincide; let us denote this common value by $\operatorname{dim} \Sigma_{V}$. Using this result, Damanik, Embree, Gorodetski, and Tcheremchantsev have shown upper and lower bounds for the dimension; see [DEGT]. A particular consequence of these bounds is the identification of the asymptotic behavior of the dimension as $V$ tends to infinity:

$$
\lim _{V \rightarrow \infty} \operatorname{dim} \Sigma_{V} \cdot \log V=\log (1+\sqrt{2}) .
$$

The paper [DEGT] also discusses some of the implications for the dynamics of the Schrödinger equation; let us mention [DT07,DT08] for further recent advances in this direction for the strongly coupled Fibonacci Hamiltonian.

By contrast, hardly anything about $\Sigma_{V}$ (beyond it having Hausdorff dimension strictly between zero and one) is known for small values of $V$. The largeness of $V$ enters the proofs of the existing results in critical ways. Consequently, these proofs indeed break down once the largeness assumption is dropped. The purpose of this paper is to fill out this gap by completely different methods.

We would like to emphasize that quantitative properties of regular Cantor sets such as thickness and denseness are widely used in dynamical systems (see [N79, N70,PT, Me]) and they have found an application in number theory (see [As00,As01,As02, Cus, Ha, $\mathrm{HI}]$ ), but to the best of our knowledge, these kinds of techniques have never been used before in the context of mathematical physics.

1.2. Statement of the main results. In this subsection we describe our results for small coupling $V$. Clearly, as $V$ approaches zero, $H_{V, \omega}$ approaches the free Schrödinger operator

$$
\left[H_{0} \psi\right](n)=\psi(n+1)+\psi(n-1),
$$

which is a well-studied object whose spectral properties are completely understood. In particular, the spectrum of $H_{0}$ is given by the interval $[-2,2]$. It is natural to ask which spectral features of $H_{V, \omega}$ approach those of $H_{0}$. It follows from Sütő's 1989 result [S89] that the Lebesgue measure of the spectrum does not extend continuously to the case $V=0$. Given this situation, one would at least hope that the dimension of the spectrum is continuous at $V=0$.

It was shown by us in [DG09a] (and independently by Cantat [Can]) that $\Sigma_{V}$ is a dynamically defined Cantor set for $V>0$ sufficiently small (i.e., the small coupling counterpart to Casdagli's result at large coupling). A consequence of this is the equality of Hausdorff dimension and upper and lower box counting dimensions of $\Sigma_{V}$ in this coupling constant regime. Our first result shows that the dimension of the spectrum indeed extends continuously to $V=0$. 
Theorem 1.1. We have

$$
\lim _{V \rightarrow 0} \operatorname{dim} \Sigma_{V}=1 .
$$

More precisely, there are constants $C_{1}, C_{2}>0$ such that

$$
1-C_{1} V \leq \operatorname{dim} \Sigma_{V} \leq 1-C_{2} V
$$

for $V>0$ sufficiently small.

We get Theorem 1.1 as a consequence of a connection between the Hausdorff dimension of a Cantor set and its denseness and thickness, along with estimates for the latter quantities. Since these notions and connections may be less familiar to at least a part of our intended audience, let us recall the definitions and some of the main results; an excellent general reference in this context is [PT].

Let $C \subset \mathbb{R}$ be a Cantor set and denote by $I$ its convex hull. Any connected component of $I \backslash C$ is called a gap of $C$. A presentation of $C$ is given by an ordering $\mathcal{U}=\left\{U_{n}\right\}_{n \geq 1}$ of the gaps of $C$. If $u \in C$ is a boundary point of a gap $U$ of $C$, we denote by $K$ the connected component of $I \backslash\left(U_{1} \cup U_{2} \cup \ldots \cup U_{n}\right)$ (with $n$ chosen so that $U_{n}=U$ ) that contains $u$ and write

$$
\tau(C, \mathcal{U}, u)=\frac{|K|}{|U|} .
$$

With this notation, the thickness $\tau(C)$ and the denseness $\theta(C)$ of $C$ are given by

$$
\tau(C)=\sup _{\mathcal{U}} \inf _{u} \tau(C, \mathcal{U}, u), \quad \theta(C)=\inf _{\mathcal{U}} \sup _{u} \tau(C, \mathcal{U}, u),
$$

and they are related to the Hausdorff dimension of $C$ by the following inequalities (cf. [PT, Sect. 4.2]),

$$
\frac{\log 2}{\log \left(2+\frac{1}{\tau(C)}\right)} \leq \operatorname{dim}_{H} C \leq \frac{\log 2}{\log \left(2+\frac{1}{\theta(C)}\right)} .
$$

Due to these inequalities, Theorem 1.1 is a consequence of the following result:

Theorem 1.2. We have

$$
\lim _{V \rightarrow 0} \tau\left(\Sigma_{V}\right)=\infty
$$

More precisely, there are constants $C_{3}, C_{4}>0$ such that

$$
C_{3} V^{-1} \leq \tau\left(\Sigma_{V}\right) \leq \theta\left(\Sigma_{V}\right) \leq C_{4} V^{-1}
$$

for $V>0$ sufficiently small.

Bovier and Ghez described in their 1995 paper [BG] the then-state of the art concerning mathematically rigorous results for Schrödinger operators in $\ell^{2}(\mathbb{Z})$ with potentials generated by primitive substitutions. The Fibonacci Hamiltonian belongs to this class; more precisely, it is in many ways the most important example within this class of models. One of the most spectacular discoveries is that, in this class of models, the spectrum jumps from being an interval for coupling $V=0$ to being a zero-measure Cantor set for coupling $V>0$. That is, as the potential is turned on, a dense set of gaps opens 


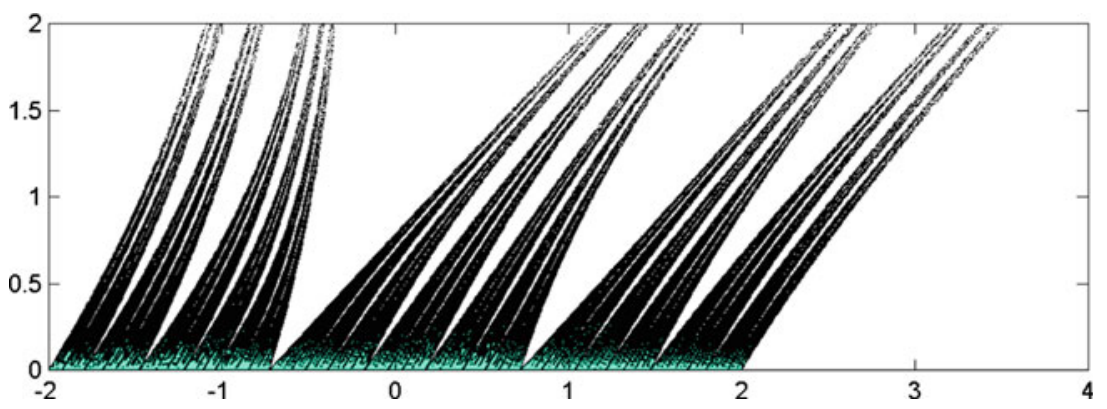

Fig. 1. The set $\left\{(E, V): E \in \Sigma_{V}, 0 \leq V \leq 2\right\}$

immediately (and the complement of these gaps has zero Lebesgue measure). It is natural to ask about the size of these gaps, which can in fact be parametrized by a canonical countable set of gap labels; see [BBG92]. These gap openings were studied in [B] for a Thue-Morse potential and in [BBG91] for period doubling potential. However, for the important Fibonacci case, the problem remained open. In fact, Bovier and Ghez write on p. 2321 of [BG]: It is a quite perplexing feature that even in the simplest case of all, the golden Fibonacci sequence, the opening of the gaps at small coupling is not known! ${ }^{1}$

Our next result resolves this issue completely and shows that, in the Fibonacci case, all gaps open linearly:

Theorem 1.3. For $V>0$ sufficiently small, the boundary points of a gap in the spectrum $\Sigma_{V}$ depend smoothly on the coupling constant $V$. Moreover, given any one-parameter continuous family $\left\{U_{V}\right\}_{V>0}$ of gaps of $\Sigma_{V}$, we have that

$$
\lim _{V \rightarrow 0} \frac{\left|U_{V}\right|}{|V|}
$$

exists and belongs to $(0, \infty)$.

Figure 1 shows a plot of the spectrum for small coupling:

The plot illustrates the results contained in Theorems 1.1-1.3. It also suggests that the $\operatorname{limit}_{V \rightarrow 0} \frac{\left|U_{V}\right|}{|V|}$ depends on the chosen family of gaps. We have more to say about the value of the limit in Theorem 1.6 below. It will turn out that its size is related to the label assigned to it by the gap labeling theorem.

Our next result concerns the sum set

$$
\Sigma_{V}+\Sigma_{V}=\left\{E_{1}+E_{2}: E_{1}, E_{2} \in \Sigma_{V}\right\}
$$

This set is equal to the spectrum of the so-called square Fibonacci Hamiltonian. Here, one considers the Schrödinger operator

$$
\begin{aligned}
{\left[H_{V}^{(2)} \psi\right](m, n)=} & \psi(m+1, n)+\psi(m-1, n)+\psi(m, n+1)+\psi(m, n-1) \\
& +V\left(\chi_{[1-\alpha, 1)}(m \alpha \bmod 1)+\chi_{[1-\alpha, 1)}(n \alpha \bmod 1)\right) \psi(m, n)
\end{aligned}
$$

1 There is a perturbative approach to this problem for a class of models that includes the Fibonacci Hamiltonian by Sire and Mosseri; see [SM89] and [OK,Si89,SM90,SMS] for related work. While their work is non-rigorous, it gives quite convincing arguments in favor of linear gap opening; see especially [SM89, Sect. 5]. It would be interesting to make their approach mathematically rigorous. 
in $\ell^{2}\left(\mathbb{Z}^{2}\right)$. The theory of tensor products of Hilbert spaces and operators then implies that $\sigma\left(H_{V}^{(2)}\right)=\Sigma_{V}+\Sigma_{V}$, see Sect. 6. This operator and its spectrum have been studied numerically and heuristically by Even-Dar Mandel and Lifshitz in a series of papers [EL06,EL07,EL08] (a similar model was studied by Sire in [Si89]). Their study suggested that at small coupling, the spectrum of $\Sigma_{V}+\Sigma_{V}$ is not a Cantor set; quite on the contrary, it has no gaps at all.

Our next theorem confirms this observation:

Theorem 1.4. For $V>0$ sufficiently small, we have that $\sigma\left(H_{V}^{(2)}\right)=\Sigma_{V}+\Sigma_{V}$ is an interval.

Certainly, the same statement holds for the cubic Fibonacci Hamiltonian (i.e., the analogously defined Schrödinger operator in $\ell^{2}\left(\mathbb{Z}^{3}\right)$ with spectrum $\left.\Sigma_{V}+\Sigma_{V}+\Sigma_{V}\right)$.

Notice that Theorem 1.4 is a consequence of Theorem 1.2 and the famous Gap Lemma, which was used by Newhouse to construct persistent tangencies and generic diffeomorphisms with an infinite number of attractors (the so-called "Newhouse phenomenon"), see Subsect. 6.2 for details:

Gap Lemma (Newhouse [N79,N70]). If $C_{1}, C_{2} \subset \mathbb{R}^{1}$ are Cantor sets such that

$$
\tau\left(C_{1}\right) \cdot \tau\left(C_{2}\right)>1,
$$

then either one of these sets is contained entirely in a gap ${ }^{2}$ of the other set, or $C_{1} \cap C_{2} \neq \emptyset$.

Let us turn to the formulation of results involving the integrated density of states, which is a quantity of fundamental importance associated with an ergodic family of Schrödinger operators. We first recall the definition of the integrated density of states. Denote the restriction of $H_{V, \omega}$ to some finite interval $\Lambda \subset \mathbb{Z}$ with Dirichlet boundary conditions by $H_{V, \omega}^{\Lambda}$. We denote by $N(E, \omega, V, \Lambda)$ the number of eigenvalues of $H_{V, \omega}^{\Lambda}$ that are less than or equal $E$. The integrated density of states is given by

$$
N(E, V)=\lim _{n \rightarrow \infty} \frac{1}{n} N(E, \omega, V,[1, n]) .
$$

We will comment on the existence of the limit and some of its basic properties in Sect. 4. One of the most important applications of the integrated density of states is the so-called gap labeling. That is, one can identify a canonical set of gap labels, that is only associated with the underlying dynamics (in this case, an irrational rotation of the circle or the shift-transformation on a substitution-generated subshift over two symbols), in such a way that the value of $N(E, V)$ for $E \in \mathbb{R} \backslash \Sigma_{V}$ must belong to this canonical set. In the Fibonacci case, this set is well-known (see, e.g., [BBG92, Eq. (6.7)]) and the general gap labeling theorem specializes to the following statement:

$$
\left\{N(E, V): E \in \mathbb{R} \backslash \Sigma_{V}\right\} \subseteq\{\{m \alpha\}: m \in \mathbb{Z}\} \cup\{1\}
$$

for every $V \neq 0$. Here $\{m \alpha\}$ denotes the fractional part of $m \alpha$, that is, $\{m \alpha\}=m \alpha-$ $\lfloor m \alpha\rfloor$. Notice that the set of gap labels is indeed $V$-independent and only depends on the value of $\alpha$ from the underlying circle rotation. Since $\alpha$ is irrational, the set of gap labels is dense. In general, a dense set of gap labels is indicative of a Cantor spectrum and hence

\footnotetext{
${ }^{2}$ For the purpose of this lemma, we also consider the two unbounded gaps in addition to the bounded gaps considered above, that is, the connected components of the complement of the convex hull of the Cantor set in question.
} 
a common (and attractive) stronger version of proving Cantor spectrum is to show that the operator "has all its gaps open." For example, the Ten Martini Problem for the almost Mathieu operator is to show Cantor spectrum, while the Dry Ten Martini Problem is to show that all labels correspond to gaps in the spectrum. The former problem has been completely solved [AJ], while the latter has not yet been completely settled. Indeed, it is in general a hard problem to show that all labels given by the gap labeling theorem correspond to gaps and there are only few results of this kind. Here we show the stronger (or "dry") form of Cantor spectrum for the weakly coupled Fibonacci Hamiltonian and establish complete gap labeling:

Theorem 1.5. There is $V_{0}>0$ such that for every $V \in\left(0, V_{0}\right]$, all gaps allowed by the gap labeling theorem are open. That is,

$$
\left\{N(E, V): E \in \mathbb{R} \backslash \Sigma_{V}\right\}=\{\{m \alpha\}: m \in \mathbb{Z}\} \cup\{1\} .
$$

Complete gap labeling for the strongly coupled Fibonacci Hamiltonian was shown by Raymond in [Ra], where he proves (3) for $V>4$. We conjecture that (3) holds for every $V>0$.

Let us return to the existence of the limit in Theorem 1.3. As was pointed out there, the value of the limit will depend on the family of gaps chosen. Now that the gap labeling has been introduced, we can refine the statement. For $m \in \mathbb{Z} \backslash\{0\}$, denote by $U_{m}(V)$ the gap of $\Sigma_{V}$ where the integrated density of states takes the value $\{m \alpha\}$.

Theorem 1.6. There is a finite constant $C^{*}$ such that for every $m \in \mathbb{Z} \backslash\{0\}$, we have

$$
\lim _{V \rightarrow 0} \frac{\left|U_{m}(V)\right|}{|V|}=\frac{C_{m}}{|m|}
$$

for a suitable $C_{m} \in\left(0, C^{*}\right)$.

Our final set of results concerns the spectral measures and transport exponents associated with the operator family. We will give precise definitions and statements of our results in Sect. 5 and limit ourselves to a brief description here. The ultimate goal of any analysis of a given Schrödinger operator is always an understanding of the associated unitary group, which then allows one to understand the dynamics of the associated time-dependent Schrödinger equation. The standard transport exponents capture the spreading of the quantum state in space. Most approaches to a study of these transport exponents proceed via (time-independent) spectral theory and link continuity properties of the spectral measure, associated to the initial state of the time evolution via the spectral theorem, to lower bounds for the transport exponents. In one space dimension, these continuity properties can in turn be investigated via an analysis of the solutions of the time-independent Schrödinger equation. Our goal is to carry this out for the weakly coupled Fibonacci Hamiltonian. Indeed, results of this kind are known, but the dependence of the quantities entering the estimates on the coupling constant had not been optimized. We revisit these approaches here and improve them to yield the best possible quantitative estimates at small coupling that can be obtained with current technology. Our results in Sect. 5 are likely not optimal and in particular do not approach the (known) zero-coupling values. We regard it as an interesting open problem to either prove or disprove that the dimension estimates for the spectral measures and the lower bounds for the transport exponents approach the values in the free (zero coupling) case as the coupling approaches zero.

Some of the results of this paper were announced in [DG09b]. 
1.3. Overview of the paper. Let us outline the remaining parts of this paper. In Sect. 2 we give some necessary background information and recall how the trace map arises in the context of the Fibonacci Hamiltonian and some of its basic properties. Moreover, since our aim is an understanding of weak coupling phenomena, we discuss the case of zero coupling. Section 3 is the heart of the paper. Here we regard the weak coupling scenario as a perturbation of zero coupling and study the dynamics of the trace map and consequences thereof for the structure of the spectrum as a set. Section 4 considers the integrated density of states and proves complete gap labeling at weak coupling and our quantitative version of the linear gap opening result. Spectral measures and transport exponents are studied by means of solution estimates in Sect. 5. Higher-dimensional models generated by a product construction are discussed in Sect. 6, where we confirm some predictions of Even-Dar Mandel and Lifshitz. Since their model is based on the off-diagonal Fibonacci Hamiltonian and the main body of this paper (and most of the other mathematical works on the Fibonacci Hamiltonian) considers the diagonal Fibonacci Hamiltonian, we develop all the basic results for the off-diagonal model in Appendix A and explain there how our work indeed confirms the predictions for the original Even-Dar Mandel-Lifshitz product model.

To assist the reader in locating the proofs of the theorems from the previous subsection, here is where they may be found: We prove Theorem 1.2 (which implies Theorem 1.1) in Subsect. 3.4, Theorem 1.3 in Subsect. 3.1, Theorem 1.4 in Subsect. 6.3, Theorem 1.5 in Subsect. 4.2, and finally Theorem 1.6 in Subsect. 4.3.

\section{Preliminaries}

2.1. Description of the trace map and previous results. The main tool that we are using here is the so-called trace map. It was originally introduced in [Ka,KKT]; further useful references include [BGJ,BR,HM, Ro]. Let us quickly recall how it arises from the substitution invariance of the Fibonacci potential; see [S87] for detailed proofs of some of the statements below.

The one step transfer matrices associated with the difference equation $H_{V, \omega} u=E u$ are given by

$$
T_{V, \omega}(m, E)=\left(\begin{array}{cc}
E-V \chi_{[1-\alpha, 1)}(m \alpha+\omega \bmod 1) & -1 \\
1 & 0
\end{array}\right) .
$$

Denote the Fibonacci numbers by $\left\{F_{k}\right\}$, that is, $F_{0}=F_{1}=1$ and $F_{k+1}=F_{k}+F_{k-1}$ for $k \geq 1$. Then, one can show that the matrices

$$
M_{-1}(E)=\left(\begin{array}{cc}
1 & -V \\
0 & 1
\end{array}\right), \quad M_{0}(E)=\left(\begin{array}{cc}
E & -1 \\
1 & 0
\end{array}\right)
$$

and

$$
M_{k}(E)=T_{V, 0}\left(F_{k}, E\right) \times \cdots \times T_{V, 0}(1, E) \quad \text { for } k \geq 1
$$

obey the recursive relations

$$
M_{k+1}(E)=M_{k-1}(E) M_{k}(E)
$$

for $k \geq 0$. Passing to the variables

$$
x_{k}(E)=\frac{1}{2} \operatorname{Tr} M_{k}(E),
$$




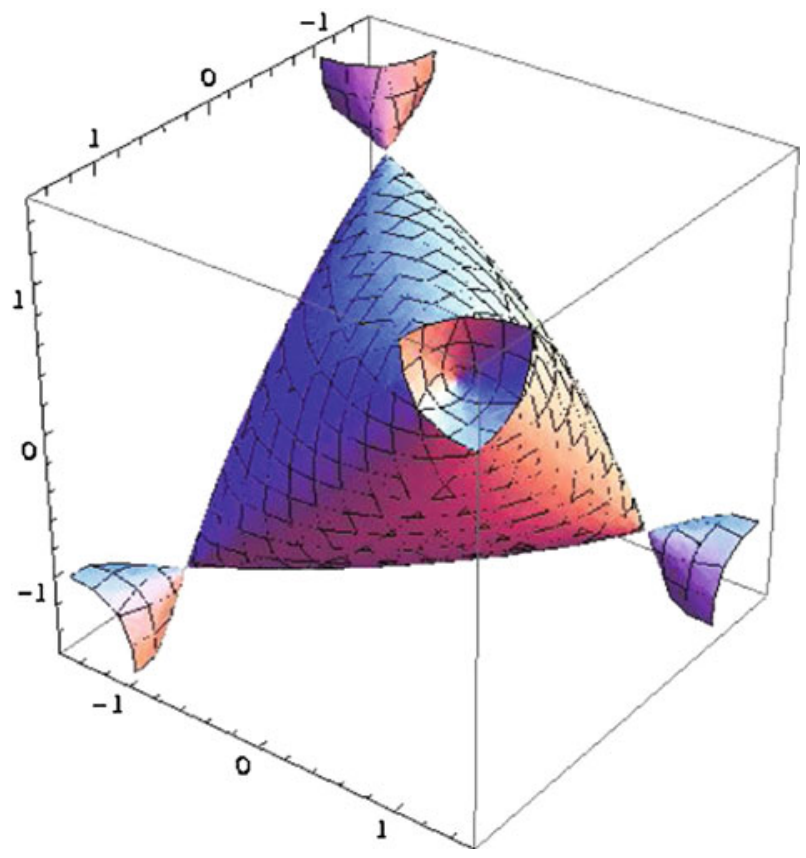

Fig. 2. The surface $S_{0.01}$

this in turn implies

$$
x_{k+1}(E)=2 x_{k}(E) x_{k-1}(E)-x_{k-2}(E) .
$$

These recursion relations exhibit a conserved quantity; namely, we have

$$
x_{k+1}(E)^{2}+x_{k}(E)^{2}+x_{k-1}(E)^{2}-2 x_{k+1}(E) x_{k}(E) x_{k-1}(E)-1=\frac{V^{2}}{4}
$$

for every $k \geq 0$.

Given these observations, it is then convenient to introduce the trace map

$$
T: \mathbb{R}^{3} \rightarrow \mathbb{R}^{3}, T(x, y, z)=(2 x y-z, x, y) .
$$

The following function ${ }^{3}$

$$
G(x, y, z)=x^{2}+y^{2}+z^{2}-2 x y z-1
$$

is invariant under the action of $T$, and hence $T$ preserves the family of cubic surfaces ${ }^{4}$

$$
S_{V}=\left\{(x, y, z) \in \mathbb{R}^{3}: x^{2}+y^{2}+z^{2}-2 x y z=1+\frac{V^{2}}{4}\right\} .
$$

Plots of the surfaces $S_{0.01}$ and $S_{0.5}$ are given in Figs. 2 and 3, respectively.

\footnotetext{
3 The function $G(x, y, z)$ is called the Fricke character, or sometimes the Fricke-Vogt invariant.

4 The surface $S_{0}$ is called the Cayley cubic.
} 


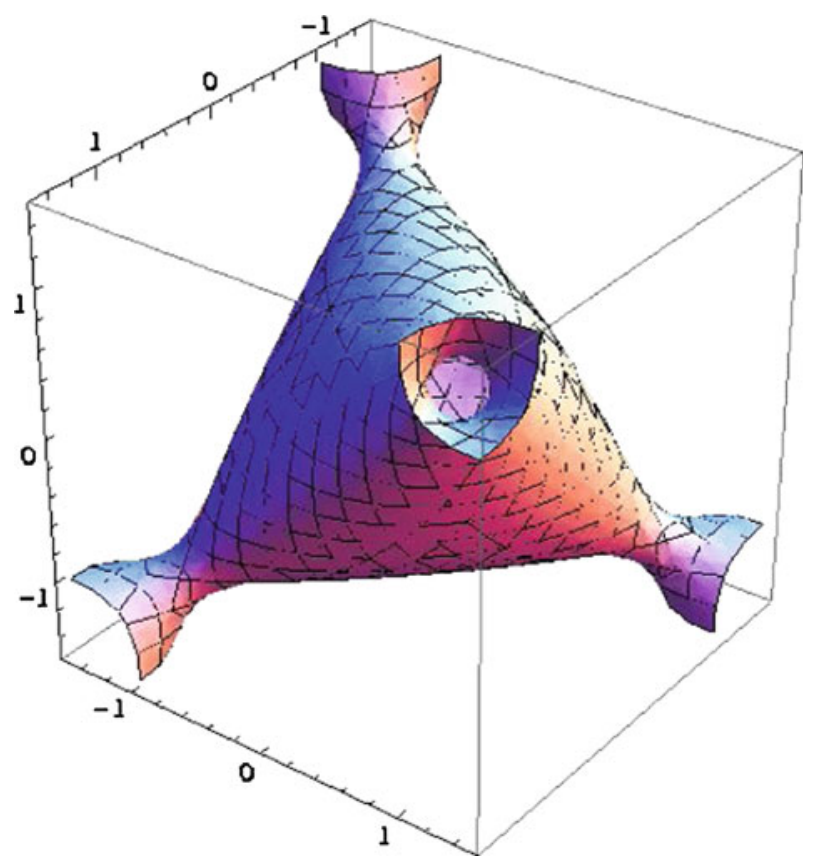

Fig. 3. The surface $S_{0.5}$

It is of course natural to consider the restriction $T_{V}$ of the trace map $T$ to the invariant surface $S_{V}$. That is, $T_{V}: S_{V} \rightarrow S_{V}, T_{V}=\left.T\right|_{S_{V}}$. Denote by $\Omega_{V}$ the set of points in $S_{V}$ whose full orbits under $T_{V}$ are bounded. A priori the set of bounded orbits of $T_{V}$ could be different from the non-wandering set ${ }^{5}$ of $T_{V}$, but our construction of the Markov partition and our analysis of the behavior of $T_{V}$ near singularities show that here these two sets do coincide. Notice that this is parallel to the construction of the symbolic coding in [Cas].

Let us recall that an invariant closed set $\Lambda$ of a diffeomorphism $f: M \rightarrow M$ is hyperbolic if there exists a splitting of the tangent space $T_{x} M=E_{x}^{u} \oplus E_{x}^{u}$ at every point $x \in \Lambda$ such that this splitting is invariant under $D f$, the differential $D f$ exponentially contracts vectors from the stable subspaces $\left\{E_{x}^{s}\right\}$, and the differential of the inverse, $D f^{-1}$, exponentially contracts vectors from the unstable subspaces $\left\{E_{x}^{u}\right\}$. A hyperbolic set $\Lambda$ of a diffeomorphism $f: M \rightarrow M$ is locally maximal if there exists a neighborhood $U$ of $\Lambda$ such that

$$
\Lambda=\bigcap_{n \in \mathbb{Z}} f^{n}(U) .
$$

We want to recall the following central result.

Theorem 2.1 ([Cas,DG09a,Can]). For $V \neq 0$, the set $\Omega_{V}$ is a locally maximal hyperbolic set of $T_{V}: S_{V} \rightarrow S_{V}$. It is homeomorphic to a Cantor set.

\footnotetext{
5 A point $p \in M$ of a diffeomorphism $f: M \rightarrow M$ is wandering if there exists a neighborhood $O(p) \subset M$ such that $f^{k}(O) \cap O=\emptyset$ for any $k \in \mathbb{Z} \backslash 0$. The non-wandering set of $f$ is the set of points that are not wandering.
} 
Denote by $\ell_{V}$ the line

$$
\ell_{V}=\left\{\left(\frac{E-V}{2}, \frac{E}{2}, 1\right): E \in \mathbb{R}\right\} .
$$

It is easy to check that $\ell_{V} \subset S_{V}$.

The second central result about the trace map we wish to recall was proven by Sütő in [S87].

Theorem 2.2 (Sütô 1987). An energy $E$ belongs to the spectrum of $H_{V, \omega}$ if and only if the positive semiorbit of the point $\left(\frac{E-V}{2}, \frac{E}{2}, 1\right)$ under iterates of the trace map $T$ is bounded.

In fact, as also shown by Sütô in [S87], the trace map can be used to generate canonical approximations of the spectrum, $\Sigma_{V}$. Namely, consider the following sets:

$\Sigma_{V}^{(n)}=\left\{E \in \mathbb{R}:\right.$ for $\left(x_{n}, y_{n}, z_{n}\right)=T^{n}\left(\frac{E-V}{2}, \frac{E}{2}, 1\right)$, we have $\left.\min \left\{\left|x_{n}\right|,\left|y_{n}\right|\right\} \leq 1\right\}$.

Then, we have $\Sigma_{V}^{(n)} \supseteq \Sigma_{V}^{(n+1)} \rightarrow \Sigma_{V}$, that is,

$$
\Sigma_{V}=\bigcap_{n \in \mathbb{Z}_{+}} \Sigma_{V}^{(n)}
$$

Figure 4 shows a plot of the sets $\left\{(E, V): E \in \Sigma_{V}^{(n)}, 0 \leq V \leq \frac{3}{4}\right\}$ for values of $n$ up to $20 .^{6}$ These plots illustrate nicely both the linear gap opening and the fact that the size of a gap depends on its label; compare Theorems 1.3 and 1.6. To further document linear gap opening through numerics, Fig. 5 zooms into a portion of Fig. 4 near a point $(E, 0)$ for an energy $E$ where a gap opens; we have chosen $E \approx 0.7248$.

2.2. Properties of the trace map for $V=0$. We will regard the case of small $V$ as a small perturbation of the case $V=0$. This subsection is devoted to the study of this "unperturbed case."

Denote by $\mathbb{S}$ the part of the surface $S_{0}$ inside the cube $\{|x| \leq 1,|y| \leq 1,|z| \leq 1\}$. The surface $\mathbb{S}$ is homeomorphic to $S^{2}$, invariant, smooth everywhere except at the four points $P_{1}=(1,1,1), P_{2}=(-1,-1,1), P_{3}=(1,-1,-1)$, and $P_{4}=(-1,1,-1)$, where $\mathbb{S}$ has conic singularities, and the trace map $T$ restricted to $\mathbb{S}$ is a factor of the hyperbolic automorphism of $\mathbb{T}^{2}=\mathbb{R}^{2} / \mathbb{Z}^{2}$ given by

$$
\mathcal{A}(\theta, \varphi)=(\theta+\varphi, \theta)(\bmod 1) .
$$

The semiconjugacy is given by the map

$$
F:(\theta, \varphi) \mapsto(\cos 2 \pi(\theta+\varphi), \cos 2 \pi \theta, \cos 2 \pi \varphi) .
$$

The map $\mathcal{A}$ is hyperbolic, and is given by the matrix $A=\left(\begin{array}{ll}1 & 1 \\ 1 & 0\end{array}\right)$, which has eigenvalues

$$
\mu=\frac{1+\sqrt{5}}{2} \quad \text { and } \quad-\mu^{-1}=\frac{1-\sqrt{5}}{2} \text {. }
$$

\footnotetext{
6 This is also how the plot in Fig. 1 was obtained. In fact, what is shown there is the set $\{(E, V): E \in$ $\left.\Sigma_{V}^{(20)}, 0 \leq V \leq 2\right\}$.
} 

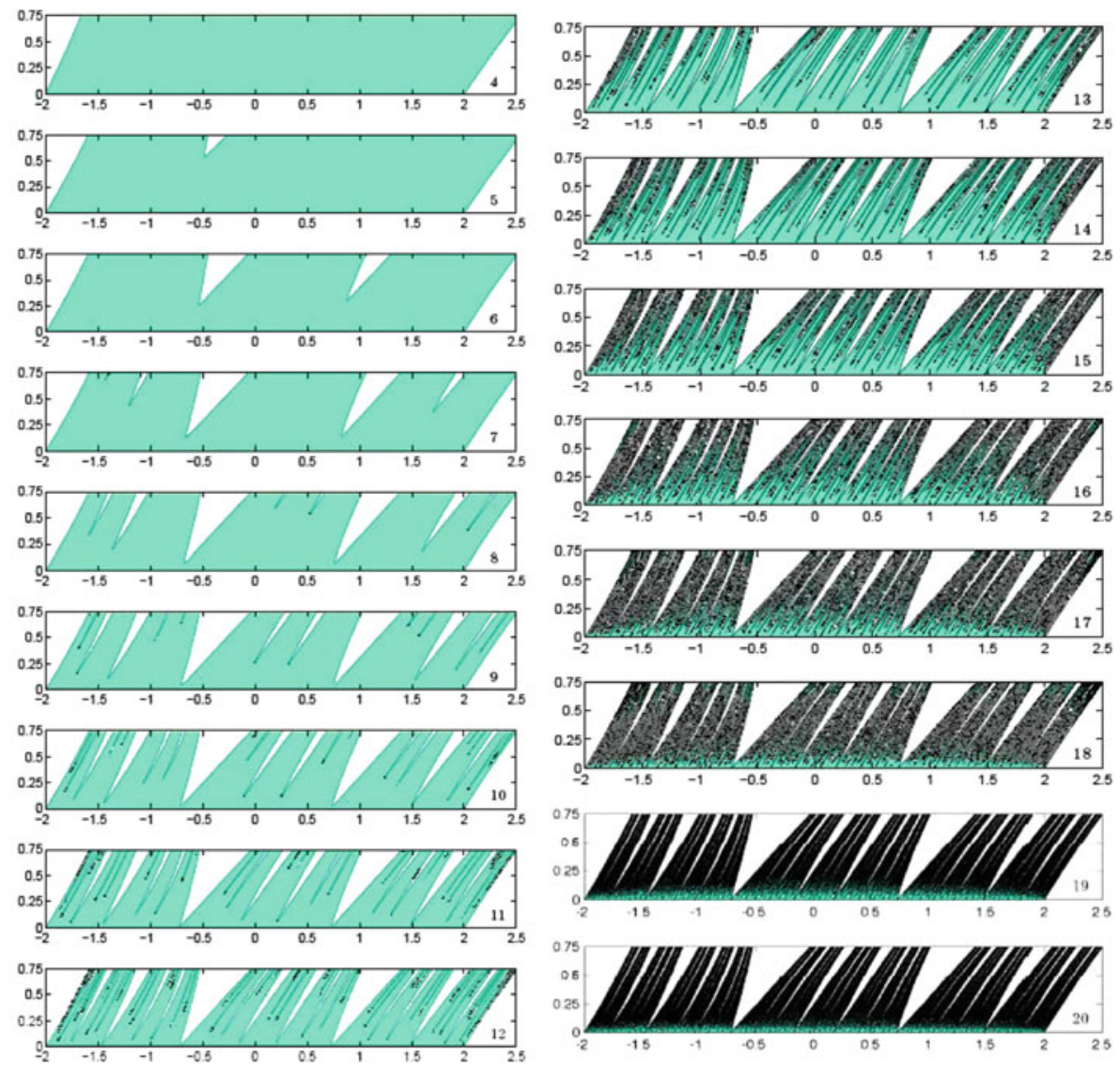

Fig. 4. The sets $\left\{(E, V): E \in \Sigma_{V}^{(n)}, 0 \leq V \leq \frac{3}{4}\right\}$ through $n=20$

Let us denote by $\mathbf{v}^{u}, \mathbf{v}^{u} \in \mathbb{R}^{2}$ the unstable and stable eigenvectors of $A$ :

$$
A \mathbf{v}^{u}=\mu \mathbf{v}^{u}, \quad A \mathbf{v}^{s}=-\mu^{-1} \mathbf{v}^{s}, \quad\left\|\mathbf{v}^{u}\right\|=\left\|\mathbf{v}^{s}\right\|=1 .
$$

Fix some small $\zeta>0$ and define the stable (resp., unstable) cone fields on $\mathbb{R}^{2}$ in the following way:

$$
\begin{aligned}
& K_{p}^{s}=\left\{\mathbf{v} \in T_{p} \mathbb{R}^{2}: \mathbf{v}=v^{u} \mathbf{v}^{u}+v^{s} \mathbf{v}^{s},\left|v^{s}\right|>\zeta^{-1}\left|v^{u}\right|\right\} \\
& K_{p}^{u}=\left\{\mathbf{v} \in T_{p} \mathbb{R}^{2}: \mathbf{v}=v^{u} \mathbf{v}^{u}+v^{s} \mathbf{v}^{s},\left|v^{u}\right|>\zeta^{-1}\left|v^{s}\right|\right\} .
\end{aligned}
$$

These cone fields are invariant:

$$
\begin{aligned}
& \forall \mathbf{v} \in K_{p}^{u} \quad A \mathbf{v} \in K_{A(p)}^{u}, \\
& \forall \mathbf{v} \in K_{p}^{s} \quad A^{-1} \mathbf{v} \in K_{A^{-1}(p)}^{s}
\end{aligned}
$$



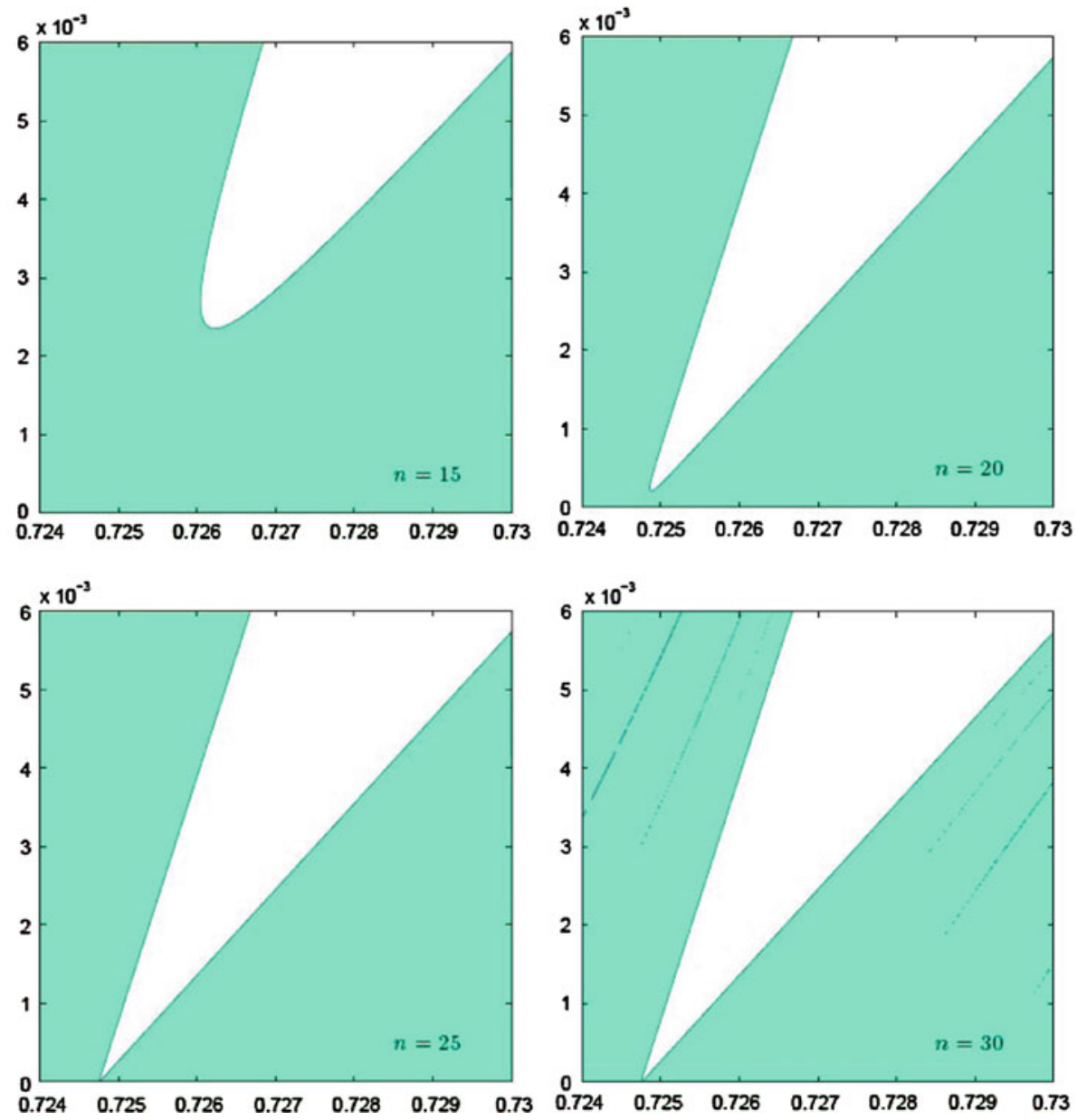

Fig. 5. The sets $\left\{(E, V): E \in \Sigma_{V}^{(n)}\right\}$ near $(0.7248,0)$ for $n=15,20,25,30$

Also, the iterates of the map $A$ expand vectors from the unstable cones, and the iterates of the map $A^{-1}$ expand vectors from the stable cones:

$$
\begin{aligned}
& \forall \mathbf{v} \in K_{p}^{u} \quad \forall n \in \mathbb{N} \quad\left|A^{n} \mathbf{v}\right|>\frac{1}{\sqrt{1+\zeta^{2}}} \mu^{n}|\mathbf{v}|, \\
& \forall \mathbf{v} \in K_{p}^{s} \quad \forall n \in \mathbb{N} \quad\left|A^{-n} \mathbf{v}\right|>\frac{1}{\sqrt{1+\zeta^{2}}} \mu^{n}|\mathbf{v}| .
\end{aligned}
$$

The families of cones $\left\{K^{s}\right\}$ and $\left\{K^{u}\right\}$ invariant under $\mathcal{A}$ can be also considered on $\mathbb{T}^{2}$.

The differential of the semiconjugacy $F$ sends these cone families to stable and unstable cone families on $\mathbb{S} \backslash\left\{P_{1}, P_{2}, P_{3}, P_{4}\right\}$. Let us denote these images by $\left\{\mathcal{K}^{s}\right\}$ and $\left\{\mathcal{K}^{u}\right\}$.

Lemma 2.3 (Lemma 3.1 from [DG09a]). The differential of the semiconjugacy DF induces a map of the unit bundle of $\mathbb{T}^{2}$ to the unit bundle of $\mathbb{S} \backslash\left\{P_{1}, P_{2}, P_{3}, P_{4}\right\}$. The 
$(0,1)$

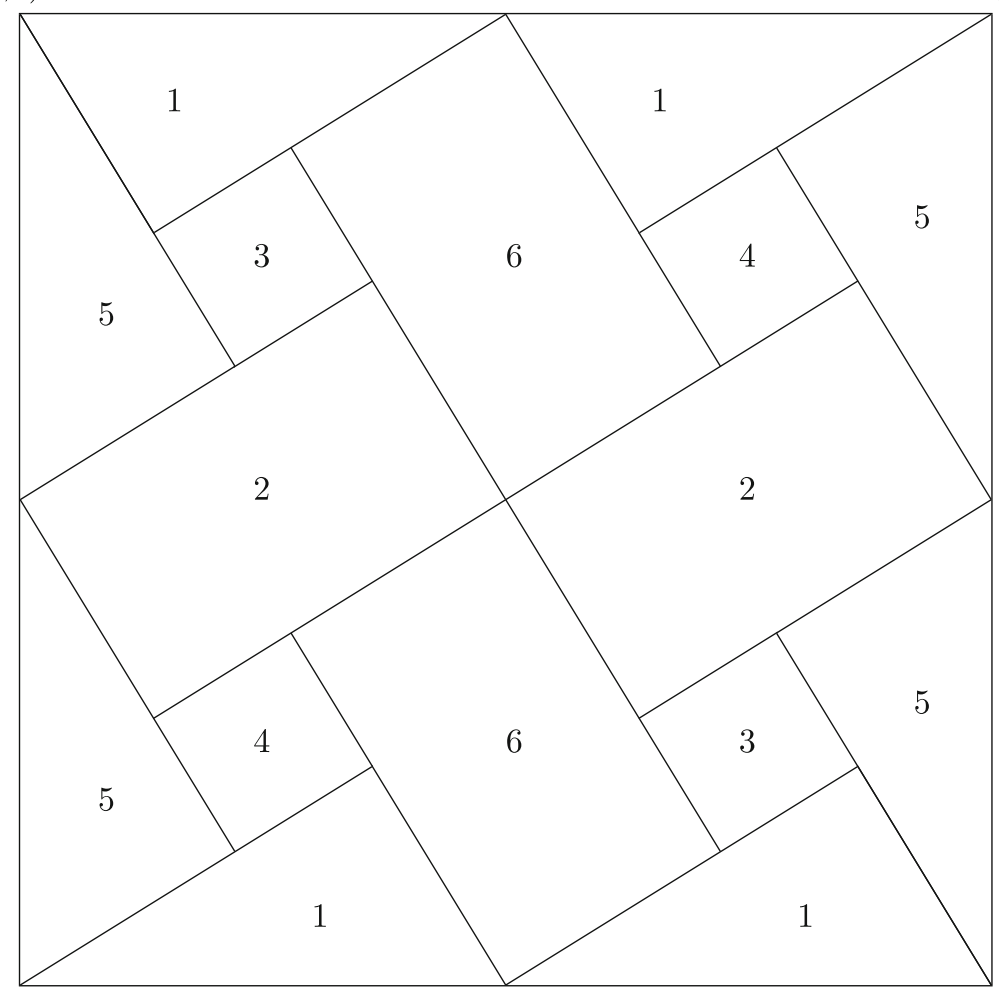

$(0,0)$

Fig. 6. The Markov partition for the map $\mathcal{A}$

derivatives of the restrictions of this map to fibers are uniformly bounded. In particular, the sizes of the cones in the families $\left\{\mathcal{K}^{s}\right\}$ and $\left\{\mathcal{K}^{u}\right\}$ are uniformly bounded away from zero.

Finally, consider the Markov partition for the map $\mathcal{A}: \mathbb{T}^{2} \rightarrow \mathbb{T}^{2}$ that is shown in Fig. 6 (and which had already appeared in [Cas]; for more details on Markov partitions for two-dimensional hyperbolic maps see [PT, App. 2]). Its image under the map $F: \mathbb{T}^{2} \rightarrow \mathbb{S}$ is a Markov partition for the pseudo-Anosov map $T: \mathbb{S} \rightarrow \mathbb{S}$.

\section{The Spectrum as a Set}

It is not hard to see that the line $\ell_{V}$ is transversal to the stable manifolds of the hyperbolic set $\Omega_{V}$ for small values of $V$ (see, e.g., [DG09a, Lemma 5.5]). Therefore the intersection of $\ell_{V}$ and $W^{s}\left(\Omega_{V}\right)$ (and, hence, $\left.\Sigma_{V}\right)$ is a dynamically defined Cantor set (see, for example, $[\mathrm{T}]$ ). In this section we study the properties of this one-parameter family of Cantor sets. Namely, in Subsect. 3.1 we prove that the size of a given gap in the Cantor set tends to zero linearly as the coupling constant (the parameter) tends to zero. In Subsect. 3.2 we use normally hyperbolic theory to introduce a normalizing coordinate system in a neighborhood of a singularity. Then in Subsect. 3.3 the order of the gaps that 


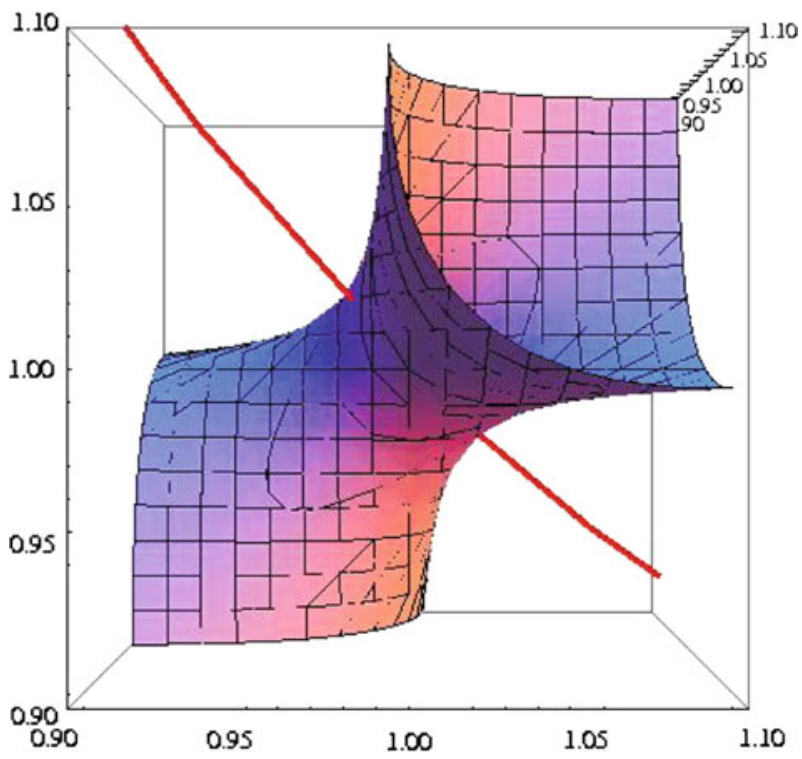

Fig. 7. $S_{0.1}$ and $\operatorname{Per}_{2}(T)$ near $(1,1,1)$

is related to the dynamics is chosen. Roughly speaking, the longer it takes for a gap to leave the union of the elements of the Markov partition, the higher is the order of the gap. Next, in Subsect. 3.5 this normalizing coordinate system is used to study the distortion properties of the transitions through a neighborhood of a singularity. Finally, in the last three subsections we bring all the pieces together and prove the distortion property that immediately implies Theorem 1.2.

\subsection{Linear gap opening as the potential is turned on. Here we prove Theorem 1.3.}

Consider the dynamics of $T$ in a neighborhood of $P_{1}=(1,1,1)$. Due to the symmetries of the trace map this will also provide information on the dynamics near the other singularities. Take $r_{0}>0$ small and let $O_{r_{0}}\left(P_{1}\right)$ be an $r_{0}$-neighborhood of the point $P_{1}=(1,1,1)$ in $\mathbb{R}^{3}$. Let us consider the set $\operatorname{Per}_{2}(T)$ of periodic points of $T$ of period 2; compare Figs. 7 and 8.

Lemma 3.1. We have

$$
\operatorname{Per}_{2}(T)=\left\{(x, y, z): x \in\left(-\infty, \frac{1}{2}\right) \cup\left(\frac{1}{2}, \infty\right), y=\frac{x}{2 x-1}, z=x\right\} \text {. }
$$

Proof. Direct calculation.

Notice that in a neighborhood $U_{1}$ of $P_{1}$, the intersection $I \equiv \operatorname{Per}_{2}(T) \cap U_{1}$ is smooth curve that is normally hyperbolic with respect to $T$ (see, e.g., App. 1 in [PT] for the formal definition of normal hyperbolicity). Therefore, the local center-stable manifold $W_{l o c}^{c s}(I)$ and the local center-unstable manifold $W_{l o c}^{c u}(I)$ defined by

$$
\begin{aligned}
& W_{\text {loc }}^{c s}(I)=\left\{p \in O_{r_{0}}\left(P_{1}\right): T^{n}(p) \in O_{r_{0}}\left(P_{1}\right) \text { for all } n \in \mathbb{N}\right\}, \\
& W_{\text {loc }}^{c u}(I)=\left\{p \in O_{r_{0}}\left(P_{1}\right): T^{-n}(p) \in O_{r_{0}}\left(P_{1}\right) \text { for all } n \in \mathbb{N}\right\}
\end{aligned}
$$




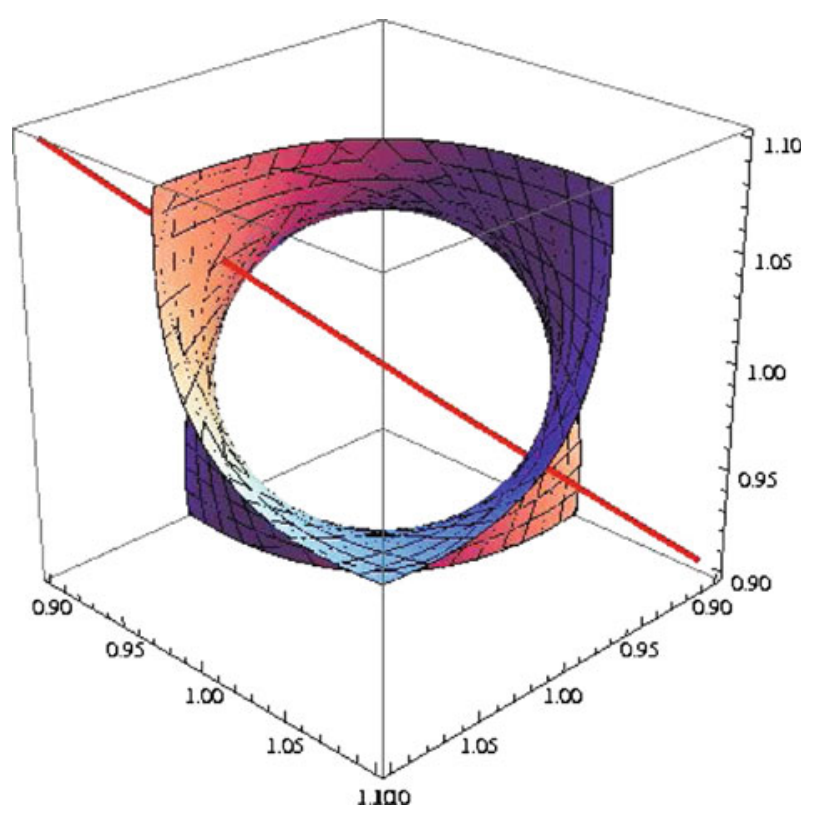

Fig. 8. $S_{0.2}$ and $\operatorname{Per}_{2}(T)$ near $(1,1,1)$

are smooth two-dimensional surfaces. Also, the local strong stable manifold $W_{l o c}^{s s}\left(P_{1}\right)$ and the local strong unstable manifold $W_{l o c}^{u u}\left(P_{1}\right)$ of the fixed point $P_{1}$, defined by

$$
\begin{aligned}
& W_{l o c}^{s s}\left(P_{1}\right)=\left\{p \in W_{l o c}^{c s}(I): T^{n}(p) \rightarrow P_{1} \text { as } n \rightarrow \infty\right\}, \\
& W_{l o c}^{u u}\left(P_{1}\right)=\left\{p \in W_{l o c}^{c u}(I): T^{-n}(p) \rightarrow P_{1} \text { as } n \rightarrow \infty\right\},
\end{aligned}
$$

are smooth curves.

The Markov partition for the pseudo-Anosov map $T: \mathbb{S} \rightarrow \mathbb{S}$ can be extended to a Markov partition for the map $T_{V}: S_{V} \rightarrow S_{V}$ for small values of $V$. Namely, there are four singular points $P_{1}=(1,1,1), P_{2}=(-1,-1,1), P_{3}=(1,-1,-1)$, and $P_{4}=(-1,1,-1)$ of $\mathbb{S}$. The point $P_{1}$ is a fixed point of $T$, and the points $P_{2}, P_{3}, P_{4}$ form a periodic orbit of period 3. For small $V$, on the surface $S_{V}$ near $P_{1}$ there is a hyperbolic orbit of the map $T_{V}=\left.T\right|_{S_{V}}$ of period 2, and near the orbit $\left\{P_{2}, P_{3}, P_{4}\right\}$ there is a hyperbolic periodic orbit of period 6. Pieces of stable and unstable manifolds of these 8 periodic points form a Markov partition for $T_{V}: S_{V} \rightarrow S_{V}$. For $V \neq 0$, the elements of this Markov partition are disjoint. Let us denote these six rectangles (the elements of the Markov partition) by $R_{V}^{1}, R_{V}^{2}, \ldots, R_{V}^{6}$. Let us also denote $R_{V}=\cup_{i=1}^{6} R_{V}^{i}$.

It is convenient now to consider $T_{V}^{6}: S_{V} \rightarrow S_{V}$ since for $T_{V}^{6}$, each of the eight periodic points that were born from singularities becomes a fixed point. Due to the symmetries of the trace map, the dynamics of $T^{6}$ is the same in a neighborhood of each of the singularities $P_{1}, P_{2}, P_{3}$, and $P_{4}$.

The set of fixed points of $T^{6}$ in a neighborhood $U_{1}$ of $P_{1}$ is a smooth curve $\operatorname{Fix}\left(T^{6}, O_{r_{0}}\left(P_{1}\right)\right)=\operatorname{Per}_{2}(T) \cap O_{r_{0}}\left(P_{1}\right)$; see Lemma 3.1 above. Each of the fixed points has one of the eigenvalues equal to 1 , one greater than 1 , and one smaller than 1 in absolute value. Therefore the curve $\operatorname{Fix}\left(T^{6}, O_{r_{0}}\left(P_{1}\right)\right)$ is a normally hyperbolic manifold, and its stable set $W^{s}\left(\operatorname{Fix}\left(T^{6}, O_{r_{0}}\left(P_{1}\right)\right)\right)$ is a smooth two dimensional surface; see 
[HPS]. The strong stable manifolds form a $C^{1}$-foliation of $W^{s}\left(\operatorname{Fix}\left(T^{6}, O_{r_{0}}\left(P_{1}\right)\right)\right)$; see [PSW, Theorem B].

If $\mathbf{p}_{V}$ and $\mathbf{q}_{V}$ are two fixed points of $T_{V}^{6}$ in $O_{r_{0}}\left(P_{1}\right)$, then these points form the intersection of the curve $\operatorname{Fix}\left(T^{6}, O_{r_{0}}\left(P_{1}\right)\right)$ with $S_{V}$ and can be found from the system

$$
\left\{\begin{array}{l}
y=\frac{x}{2 x-1} \\
z=x \\
x^{2}+y^{2}+z^{2}-2 x y z=1+\frac{V^{2}}{4} .
\end{array}\right.
$$

If we parameterize $\operatorname{Fix}\left(T^{6}, O_{r_{0}}\left(P_{1}\right)\right)$ as $\left\{x=t+1, z=t+1, y=\frac{t+1}{2 t+1}\right\}$, we get

$$
(t+1)^{2}+\frac{t+1}{2 t+1}+(t+1)^{2}-2(t+1)^{2} \frac{t+1}{2 t+1}=1+\frac{V^{2}}{4},
$$

or

$$
\frac{4 t^{4}+10 t^{3}+9 t^{2}+4 t+1}{(2 t+1)^{2}}=1+\frac{V^{2}}{4} .
$$

Since for the function $f(t)=\frac{4 t^{4}+10 t^{3}+9 t^{2}+4 t+1}{(2 t+1)^{2}}$, we have $f(0)=1, f^{\prime}(0)=0, f^{\prime \prime}(0)>0$, the distance between the points $\mathbf{p}_{V}$ and $\mathbf{q}_{V}$ is of order $|V|$ for small values of $V$.

Lemma 3.2. Let $W \subset \mathbb{R}^{3}$ be a smooth surface with a $C^{1}$-foliation on it. Let $\xi \subset W$ be a smooth curve transversal to the foliation. Fix a leaf $L \subset W$ of the foliation, and denote $P=L \cap \xi$. Take a point $Q \neq P, Q \in L$, and a line $\ell_{0} \subset \mathbb{R}^{3}, Q \in \ell_{0}$, tangent to $W$ at $Q$, but not tangent to the leaf L. Suppose that a family of lines $\left\{\ell_{V}\right\}_{V \in\left(0, V_{0}\right)}$ is given such that $\ell_{V} \rightarrow \ell_{0}$ as $V \rightarrow 0$, each line $\ell_{V}, V>0$, intersects $W$ at two points $p_{V}$ and $q_{V}$, and $p_{V} \rightarrow Q, q_{V} \rightarrow Q$ as $V \rightarrow 0$.

Denote by $L_{p_{V}}$ and $L_{q_{V}}$ the leaves of the foliation that contain $p_{V}$ and $q_{V}$, respectively. Denote $\mathbf{p}_{V}=L_{p_{V}} \cap \xi$ and $\mathbf{q}_{V}=L_{q_{V}} \cap \xi$. Then there exists a finite non-zero limit

$$
\lim _{V \rightarrow 0} \frac{\operatorname{dist}\left(p_{V}, q_{V}\right)}{\operatorname{dist}\left(\mathbf{p}_{V}, \mathbf{q}_{V}\right)} .
$$

Proof. Since $\ell_{0}$ is not tangent to the leaf $L$, there exists a curve $\widetilde{\xi} \subset W$ tangent to $\ell_{0}$, transversal to the foliation, and such that $Q \in \widetilde{\xi}$.

Set $\widetilde{\mathbf{p}}=L_{p_{V}} \cap \widetilde{\xi}, \widetilde{\mathbf{q}}=L_{q_{V}} \cap \widetilde{\xi}$. Since the foliation is $C^{1}$, there exists a finite non-zero limit

$$
\lim _{V \rightarrow 0} \frac{\operatorname{dist}\left(\mathbf{p}_{V}, \mathbf{q}_{V}\right)}{\operatorname{dist}\left(\widetilde{\mathbf{p}}_{V}, \widetilde{\mathbf{q}}_{V}\right)} \neq 0 .
$$

Let us consider a plane $\Pi$ tangent to $W$ at $Q$, and let $\pi: W \rightarrow \Pi$ be an orthogonal projection (well defined and smooth in a neighborhood of $Q$ ). It is clear that

$$
\lim _{V \rightarrow 0} \frac{\operatorname{dist}\left(\pi\left(\widetilde{\mathbf{p}}_{V}\right), \pi\left(\widetilde{\mathbf{q}}_{V}\right)\right)}{\operatorname{dist}\left(\widetilde{\mathbf{p}}_{V}, \widetilde{\mathbf{q}}_{V}\right)}=1 .
$$

Also, since $\ell_{V} \rightarrow \ell_{0}$ as $V \rightarrow 0$, we have

$$
\lim _{V \rightarrow 0} \frac{\operatorname{dist}\left(\pi\left(p_{V}\right), \pi\left(q_{V}\right)\right)}{\operatorname{dist}\left(p_{V}, q_{V}\right)}=1 .
$$


Finally, since $\pi$ sends the $C^{1}$-foliation of $W$ to a $C^{1}$-foliation on $\Pi$, the projection along this foliation from $\pi\left(\ell_{V}\right)$ to $\ell_{0}$ is $C^{1}$-close to isometry. In particular,

$$
\lim _{V \rightarrow 0} \frac{\operatorname{dist}\left(\pi\left(p_{V}\right), \pi\left(q_{V}\right)\right)}{\operatorname{dist}\left(\pi\left(\widetilde{\mathbf{p}}_{V}\right), \pi\left(\widetilde{\mathbf{q}}_{V}\right)\right)}=1 .
$$

The statement of Lemma 3.2 follows now from (5)-(8).

Proof of Theorem 1.3. Pick any bounded gap in the spectrum and the corresponding gap $O$ in $\ell_{V} \backslash W^{s}\left(\Omega_{V}\right)$. The boundary points $p_{V}$ and $q_{V}$ of $O$ belong to stable manifolds of two fixed points near one of the singularities $P_{1}, P_{2}, P_{3}$, or $P_{4}$. Without loss of generality assume that those fixed points are $\mathbf{p}_{V}$ and $\mathbf{q}_{V}$ in a neighborhood of $P_{1}$. The surface $W=W^{s}\left(\operatorname{Fix}\left(T^{6}, O_{r_{0}}\left(P_{1}\right)\right)\right)$ is $C^{1}$-foliated by strong stable manifolds of fixed points from $\operatorname{Fix}\left(T^{6}, O_{r_{0}}\left(P_{1}\right)\right)$, and the curve $\xi=\operatorname{Fix}\left(T^{6}, O_{r_{0}}\left(P_{1}\right)\right)$ is transversal to this foliation. Therefore Lemma 3.2 implies that there exists a finite non-zero limit $\lim _{V \rightarrow 0} \frac{\operatorname{dist}\left(p_{V}, q_{V}\right)}{\operatorname{dist}\left(\mathbf{p}_{V}, \mathbf{q}_{V}\right)}$, and, since $\operatorname{dist}\left(\mathbf{p}_{V}, \mathbf{q}_{V}\right)$ is of order $|V|$ for small values of $V$, this implies Theorem 1.3.

3.2. Choice of a coordinate system in a neighborhood of a singular point. Due to the smoothness of the invariant manifolds of the curve of periodic points of period two described in Sect. 3.1, there exists a smooth change of coordinates $\Phi: O_{r_{0}}\left(P_{1}\right) \rightarrow \mathbb{R}^{3}$ such that $\Phi\left(P_{1}\right)=(0,0,0)$ and

- $\Phi(I)$ is a part of the line $\{x=0, z=0\}$;

- $\Phi\left(W_{l o c}^{c s}(I)\right)$ is a part of the plane $\{z=0\}$;

- $\Phi\left(W_{l o c}^{c u}(I)\right)$ is a part of the plane $\{x=0\}$;

- $\Phi\left(W_{l o c}^{s s}\left(P_{1}\right)\right)$ is a part of the line $\{y=0, z=0\}$;

- $\Phi\left(W_{l o c}^{u u}\left(P_{1}\right)\right)$ is a part of the line $\{x=0, y=0\}$.

Denote $f=\Phi \circ T \circ \Phi^{-1}$.

In this case,

$$
A \equiv D f(0,0,0)=D\left(\Phi \circ T \circ \Phi^{-1}\right)(0,0,0)=\left(\begin{array}{lll}
\lambda^{-1} & 0 & 0 \\
0 & -1 & 0 \\
0 & 0 & \lambda
\end{array}\right)
$$

where $\lambda$ is the largest eigenvalue of the differential $D T\left(P_{1}\right): T_{P_{1}} \mathbb{R}^{3} \rightarrow T_{P_{1}} \mathbb{R}^{3}$,

$$
D T\left(P_{1}\right)=\left(\begin{array}{lll}
2 & 2 & -1 \\
1 & 0 & 0 \\
0 & 1 & 0
\end{array}\right), \quad \lambda=\frac{3+\sqrt{5}}{2}=\mu^{2} .
$$

Let us denote $\mathfrak{S}_{V}=\Phi\left(\mathfrak{S}_{V}\right)$. Then, away from $(0,0,0)$, the family $\left\{\mathfrak{S}_{V}\right\}$ is a smooth family of surfaces, $\mathfrak{S}_{0}$ is diffeomorphic to a cone, contains the lines $\{y=0, z=0\}$ and $\{x=0, y=0\}$, and at each non-zero point on these lines, it has a quadratic tangency with a horizontal or vertical plane.

Due to the symmetries of the trace map, similar changes of coordinates exist in a neighborhood of each of the other singularities. Denote $O_{r_{0}}=O_{r_{0}}\left(P_{1}\right) \cup O_{r_{0}}\left(P_{2}\right) \cup$ $O_{r_{0}}\left(P_{3}\right) \cup O_{r_{0}}\left(P_{4}\right)$. 
Fix a small constant $\mathbf{C}>0$ and introduce the following cone fields in $\mathbb{R}^{3}$ :

$$
\begin{aligned}
& \mathbf{K}_{p}^{u}=\left\{\mathbf{v} \in T_{p} \mathbb{R}^{3}, \mathbf{v}=\mathbf{v}_{x y}+\mathbf{v}_{z}:\left|\mathbf{v}_{z}\right|>\mathbf{C} \sqrt{\left|z_{p}\right|}\left|\mathbf{v}_{x y}\right|\right\}, \\
& \widetilde{\mathbf{K}}_{p}^{u}=\left\{\mathbf{v} \in T_{p} \mathbb{R}^{3}, \mathbf{v}=\mathbf{v}_{x}+\mathbf{v}_{y z}:\left|\mathbf{v}_{z}\right|>\mathbf{C}^{-1}\left|\mathbf{v}_{x y}\right|\right\}, \\
& \mathbf{K}_{p}^{s}=\left\{\mathbf{v} \in T_{p} \mathbb{R}^{3}, \mathbf{v}=\mathbf{v}_{x}+\mathbf{v}_{y z}:\left|\mathbf{v}_{x}\right|>\mathbf{C} \sqrt{\left|x_{p}\right|}\left|\mathbf{v}_{y z}\right|\right\}, \\
& \widetilde{\mathbf{K}}_{p}^{s}=\left\{\mathbf{v} \in T_{p} \mathbb{R}^{3}, \mathbf{v}=\mathbf{v}_{z}+\mathbf{v}_{x y}:\left|\mathbf{v}_{x}\right|>\mathbf{C}^{-1}\left|\mathbf{v}_{y z}\right|\right\} .
\end{aligned}
$$

Lemma 3.3. There are $r_{1} \in\left(0, r_{0}\right)$ and $m_{0} \in \mathbb{N}$ such that the following holds.

1. $T^{m_{0}}\left(\ell_{0}\right) \cap O_{r_{1}}$ is a union of two connected curves $\gamma_{1}$ and $\gamma_{2}$, and $\Phi\left(\gamma_{i}\right), i=1,2$, is tangent to the cone field $\widetilde{\mathbf{K}}^{u}$;

2. $F^{-1}\left(T^{m_{0}}\left(\ell_{0} \cap \mathbb{S}\right)\right)$ is tangent to the cone field $K^{u}$ (defined by (4)).

Definition 3.4. We will call the rectangle $R^{6}$ (the element of the Markov partition) the opposite to singularities $P_{1}$ and $P_{3}$, and we will call the rectangle $R^{5}$ the opposite to singularities $P_{2}$ and $P_{4}$

Notice that $T^{m_{0}}\left(\ell_{0} \cap \mathbb{S}\right)$ consists of a curve that connects $P_{1}$ with a stable boundary of $R_{6}$, a finite number of curves $\xi_{i}$ that connect two stable sides of an element of the Markov partition and such that $F^{-1}\left(\xi_{i}\right)$ is tangent to the cone field $K^{u}$, and a curve that connects some other singularity with a stable boundary of its opposite rectangle.

Now let us take $r_{2} \in\left(0, r_{1}\right)$ so small that $\cup_{i=-3}^{3} T^{i}\left(O_{r_{2}}\right) \subset O_{r_{1}}$.

For small $V$, denote by $\mathbb{S}_{V, O_{r_{2}}}$ the bounded component of $S_{V} \backslash O_{r_{2}}$. The family $\left\{\mathbb{S}_{V, O_{r_{2}}}\right\}_{V \in\left[0, V_{0}\right)}$ of surfaces with boundary depends smoothly on the parameter and has uniformly bounded curvature. For small $V$, a projection $\pi_{V}: \mathbb{S}_{V, O_{r_{2}}} \rightarrow \mathbb{S}$ is defined. The map $\pi_{V}$ is smooth, and if $p \in \mathbb{S}, q \in \mathbb{S}_{V, O_{r_{2}}}$, and $\pi_{V}(q)=p$, then $T_{p} \mathbb{S}$ and $T_{q} S_{V}$ are close. Denote by $\mathcal{K}_{V}^{u}$ (resp., $\mathcal{K}_{V}^{s}$ ) the image of the cone $\mathcal{K}^{u}$ (resp., $\mathcal{K}^{s}$ ) under the differential of $\pi_{V}^{-1}$.

Denote $F_{V}=\pi_{V}^{-1} \circ F, \quad F_{V}: F^{-1}\left(\mathbb{S} \backslash O_{r_{2}}\right) \rightarrow S_{V}$. Denote also $\Psi_{V}=\Phi \circ F_{V}$. Compactness and Mean Value Theorem type arguments imply the following statement.

Lemma 3.5. There are $V_{0}>0$ and $\widetilde{C}>0$ such that the following holds. Suppose that $a, b \in \mathbb{T}^{2} \backslash F^{-1}\left(O_{r_{2}}\right), v_{a} \in T_{a} \mathbb{T}^{2}, v_{b} \in T_{b} \mathbb{T}^{2}$. Then the following inequalities hold for all $V \in\left[0, V_{0}\right]:$

$$
\begin{aligned}
\operatorname{dist}\left(F_{V}(a), F_{V}(b)\right) & \leq \widetilde{C} \operatorname{dist}(a, b), \\
\operatorname{dist}(a, b) & \leq \widetilde{C} \operatorname{dist}\left(F_{V}(a), F_{V}(b)\right), \\
\angle\left(D F_{V}\left(v_{a}\right), D F_{V}\left(v_{b}\right)\right) & \leq \widetilde{C}\left(\angle\left(v_{a}, v_{b}\right)+\operatorname{dist}(a, b)\right), \\
\angle\left(v_{a}, v_{b}\right) & \leq \widetilde{C}\left(\angle\left(D F_{V}\left(v_{a}\right), D F_{V}\left(v_{b}\right)\right)+\operatorname{dist}\left(F_{V}(a), F_{V}(b)\right)\right) .
\end{aligned}
$$

Moreover, if $\Psi_{V}(a)$ and $\Psi_{V}(b)$ are defined, then

$$
\begin{aligned}
\operatorname{dist}\left(\Psi_{V}(a), \Psi_{V}(b)\right) & \leq \widetilde{C} \operatorname{dist}(a, b) \\
\operatorname{dist}(a, b) & \leq \widetilde{C} \operatorname{dist}\left(\Psi_{V}(a), \Psi_{V}(b)\right) \\
\angle\left(D \Psi_{V}\left(v_{a}\right), D \Psi_{V}\left(v_{b}\right)\right) & \leq \widetilde{C}\left(\angle\left(v_{a}, v_{b}\right)+\operatorname{dist}(a, b)\right) \\
\angle\left(v_{a}, v_{b}\right) & \leq \widetilde{C}\left(\angle\left(D \Psi_{V}\left(v_{a}\right), D \Psi_{V}\left(v_{b}\right)\right)+\operatorname{dist}\left(\Psi_{V}(a), \Psi_{V}(b)\right)\right) .
\end{aligned}
$$


Finally, notice that if $\mathbf{C}$ and $V_{0}$ are taken sufficiently small, then the cone fields $K^{u}$ on $\mathbb{T}^{2}$ and $\mathbf{K}^{u}, \widetilde{\mathbf{K}}^{u}$ respect each other in the following sense. Suppose that $a \in \mathbb{T}^{2} \backslash F^{-1}\left(O_{r_{2}}\right)$ is such that $\Psi_{V}(a)$ is defined for $V \in\left[0, V_{0}\right]$, and $v_{a} \in K_{a}^{u}$. Lemma 2.3 implies that

$$
D \Psi_{V}\left(v_{a}\right) \in \mathbf{K}_{\Psi_{V}(a)}^{u} .
$$

On the other hand, if $b \in \mathbb{T}^{2} \backslash F^{-1}\left(O_{r_{2}}\right)$ is such that $\Psi_{V}(b)$ is defined and $D \Psi_{V}\left(v_{b}\right) \in$ $\widetilde{\mathbf{K}}_{\Psi_{V}(b)}^{u}$, then

$$
v_{b} \in K_{b}^{u}
$$

3.3. Ordering of the gaps. To estimate the thickness of a Cantor set from below (or the denseness from above), it is enough to consider one particular ordering of its gaps. Here we choose a convenient ordering of gaps in $\ell_{V} \cap W^{s}\left(\Omega_{V}\right)$ (which is affine equivalent to $\Sigma_{V}$ ).

The trace map $T_{V}, V \neq 0$, has two periodic points of period 2, denote them by $P_{1}(V)$ and $P_{1}^{\prime}(V)$, and six periodic points of period 6, denote them by $P_{2}(V), P_{2}^{\prime}(V), P_{3}(V)$, $P_{3}^{\prime}(V), P_{4}(V)$, and $P_{4}^{\prime}(V)$. In Sect. 3.1 we showed that the distance between $P_{i}(V)$ and $P_{i}^{\prime}(V)$ is of order $|V|$.

We can choose the notation (swapping the notation for $P_{1}(V)$ and $P_{1}^{\prime}(V)$, and/or for $P_{2}(V)$ and $P_{2}^{\prime}(V)$ if necessary) in such a way that the following lemma holds.

Lemma 3.6. If $V$ is small enough, the line $\ell_{V}$ contains points $B_{1}(V) \in \ell_{V} \cap W^{s s}\left(P_{1}(V)\right)$ and $B_{2}(V) \in \ell_{V} \cap W^{s s}\left(P_{2}(V)\right)$ such that every point of the line which is not between $B_{1}(V)$ and $B_{2}(V)$ tends to infinity under iterates of $T_{V}$.

Denote by $\mathbf{l}_{V}$ the closed interval on $\ell_{V}$ between the points $B_{1}(V)$ and $B_{2}(V)$. It is known that the set of points on $\mathbf{l}_{V}$ with bounded positive semiorbits is a dynamically defined Cantor set; see [DG09a, Can]. We would like to estimate the thickness of this Cantor set.

Lemma 3.7. There are $m_{0} \in \mathbb{N}, 0<C_{1}<C_{2}$ and $V_{0}>0$ such that for all $V \in\left[0, V_{0}\right]$ the following holds:

1. $T_{V}^{m_{0}}\left(\mathbf{l}_{V}\right) \cap O_{r_{1}}$ is a union of two connected curves $\gamma_{1}(V)$ and $\gamma_{2}(V)$, and $\Phi\left(\gamma_{i}(V)\right), i=1,2$, is tangent to the cone field $\widetilde{\mathbf{K}}^{u}$;

2. $F_{V}^{-1}\left(T^{m_{0}}\left(\mathbf{l}_{V}\right) \backslash O_{r_{2}}\right)$ is tangent to the cone field $K^{u}$;

3. $T_{V}^{m_{0}}\left(\mathbf{l}_{V}\right)$ consists of a curve that connects $W_{\text {loc }}^{\text {ss }}\left(P_{1}\right)$ with a stable boundary of $R^{6}, a$ finite number of curves $\xi_{i}(V)$, each of which connects two stable sides of an element of the Markov partition and is such that $F_{V}^{-1}\left(\xi_{i}\right)$ is tangent to the cone field $K^{u}, a$ curve that connects $W_{\text {loc }}^{\text {ss }}\left(P_{i}\right), i \in\{2,3,4\}$, with a stable boundary of an opposite rectangle to $P_{i}$, and some "gaps" between the curves mentioned above. The length of these "gaps" is between $C_{1} V$ and $C_{2} V$ for all small enough $V$.

Proof. The statement holds for $V=0$ (see Lemma 3.3). For $V$ positive but small enough Properties 1 and 2 hold by continuity, and Property 3 follows from the fact that the distances between finite pieces of strong stable manifolds of points $\mathbf{p}_{V}$ and $\mathbf{q}_{V}$ is of order $V$, and these strong stable manifolds form the stable parts of boundary of the Markov partition for $T_{V}$. 
We will call the preimages (under $T_{V}^{m_{0}}$ ) of the gaps defined in Lemma 3.7 the gaps of order 1 .

Definition 3.8. A smooth curve $\gamma \subset S_{V}$ is tangent to an unstable cone field if $F_{V}^{-1}\left(\gamma \backslash O_{r_{2}}\right)$ is tangent to $K^{u}$, and $\Phi\left(\gamma \cap O_{r_{1}}\right)$ is tangent to $\mathbf{K}^{u}$.

Definition 3.9. A curve is of type one if it is tangent to the unstable cone field and connects opposite sides of stable boundaries of some $R^{i}$.

A curve is of type two if it is tangent to the unstable cone field and connects a point from $W_{\text {loc }}^{\text {ss }}\left(P_{i}\right), i \in\{1,2,3,4\}$, with a stable boundary of an opposite element of the Markov partition.

In this terminology, Lemma 3.7 claims that $T_{V}^{m_{0}}\left(\mathbf{l}_{V}\right)$ consists of two curves of type two, some curves of type one, and some gaps between them of size of order $V$.

Lemma 3.10. An image of a curve of type one under $T^{6}$ is a union of a finite number of curves of type one, and of a finite number of gaps of length between $C_{1} V$ and $C_{2} V$. An image of a curve of type two under $T^{6}$ is a union of a curve of type two, a finite number of curves of type one, and a finite number of gaps of length between $C_{1} V$ and $C_{2} V$.

Proof. The first part follows from the properties of the Markov partition and the fact that the distance between strong stable (strong unstable) manifolds that form the Markov partition is of order $V$, see Subsect. 3.1. An image of a curve tangent to an unstable cone field is a curve tangent to an unstable cone field. Also, $T^{6}\left(W_{l o c}^{s s}\left(P_{i}\right)\right) \subset W_{l o c}^{s s}\left(P_{i}\right)$. Therefore the image of a curve of type two under $T^{6}$ is a curve which is close to a finite piece of a strong unstable manifold of $P_{i}$, so the second part follows.

Suppose that the gaps of order $k$ have already been defined. Consider the complement of all gaps of order not greater than $k$ on $\mathbf{l}_{V}$. It consists of a finite number of closed intervals. Let $J$ be one of them. Consider the curve $T_{V}^{m_{0}+6(k-1)}(J)$. By construction, it is either a curve of type one, or of type two. In either case, the image $T_{V}^{6}\left(T_{V}^{m_{0}+6(k-1)}(J)\right)=T_{V}^{m_{0}+6 k}(J)$ consists of some curves of type one or two, and some gaps of size $\sim V$. Let us say that the preimages of these gaps (under $T_{V}^{m_{0}+6 k}$ ) are gaps of order $k+1$. It is clear that every gap in $\mathbf{l}_{V} \cap W^{s}\left(\Omega_{V}\right)$ has some finite order. Therefore we have ordered all the gaps.

3.4. Distortion property: estimate of the gap sizes. Let us consider $\mathbf{l}_{V}$ and some gap $\gamma_{G} \subset \mathbf{l}_{V}$ of order $n$. A bridge that corresponds to this gap is a connected component of the complement of the union of all gaps of order $\leq n$ next to the gap. There are two bridges that correspond to the chosen gap; take one of them, and denote it by $\gamma_{B}$. Denote also $\gamma=\gamma_{G} \cup \gamma_{B}$. Now let us consider $\Gamma_{G} \equiv T_{V}^{m_{0}+6(n-1)}\left(\gamma_{G}\right)$ and $\Gamma_{B} \equiv T_{V}^{m_{0}+6(n-1)}\left(\gamma_{B}\right)$. By definition of the order $n$ of the gap we know that

$$
C_{3} V \leq \frac{\left|\Gamma_{G}\right|}{\left|\Gamma_{B}\right|} \leq C_{4} V
$$

for some constants $C_{3}$ and $C_{4}$ independent of $V$.

Proposition 3.11. There is a constant $K>1$ independent of the choice of the gap and of $V$ such that

$$
K^{-1} \frac{\left|\gamma_{G}\right|}{\left|\gamma_{B}\right|} \leq \frac{\left|\Gamma_{G}\right|}{\left|\Gamma_{B}\right|} \leq K \frac{\left|\gamma_{G}\right|}{\left|\gamma_{B}\right|} .
$$


Notice that Theorem 1.2 immediately follows from Proposition 3.11.

The rest of this section is devoted to the proof of Proposition 3.11, which is completed in Subsect. 3.7.

3.5. Dynamics near singularities. Here we prove several technical propositions on the properties of the trace map in the coordinate system constructed in Subsect. 3.2. The first two propositions are reformulations of [DG09a, Prop. 1]. The first one claims that a certain unstable cone field is invariant.

We will use the variables $(x, y, z)$ for coordinates in $\mathbb{R}^{3}$. For a point $p \in \mathbb{R}^{3}$, we will denote its coordinates by $\left(x_{p}, y_{p}, z_{p}\right)$.

Proposition 3.12. Given $C_{1}>0, C_{2}>0, \lambda>1$, there exists $\delta_{0}=\delta_{0}\left(C_{1}, C_{2}, \lambda\right)$ such that for any $\delta \in\left(0, \delta_{0}\right)$, the following holds.

Let $f: \mathbb{R}^{3} \rightarrow \mathbb{R}^{3}$ be a $C^{2}$-diffeomorphism such that

(i) $\|f\|_{C^{2}} \leq C_{1}$;

(ii) The plane $\{z=0\}$ is invariant under iterates of $f$;

(iii) $\|D f(p)-A\|<\delta$ for every $p \in \mathbb{R}^{3}$, where

$$
A=\left(\begin{array}{ccc}
\lambda^{-1} & 0 & 0 \\
0 & 1 & 0 \\
0 & 0 & \lambda
\end{array}\right)
$$

is a constant matrix.

Introduce the following cone field in $\mathbb{R}^{3}$ :

$$
K_{p}^{u}=\left\{\mathbf{v} \in T_{p} \mathbb{R}^{3}, \mathbf{v}=\mathbf{v}_{x y}+\mathbf{v}_{z}:\left|\mathbf{v}_{z}\right| \geq C_{2} \sqrt{\left|z_{p}\right|}\left|\mathbf{v}_{x y}\right|\right\} .
$$

Then for any point $p=\left(x_{p}, y_{p}, z_{p}\right),\left|z_{p}\right| \leq 1$ we have

$$
D f\left(K_{p}\right) \subseteq K_{f(p)}^{u} .
$$

Notice that the choice of the cone field $K_{p}^{u}$ here (in (13)) and below (in (18)) corresponds to the choice of the cone field $\mathbf{K}_{p}^{u}$ in (9).

The next proposition establishes expansion of vectors from the introduced unstable cones under the differential of the map.

Proposition 3.13. Given $C_{1}>0, C_{2}>0, \lambda>1, \varepsilon \in\left(0, \frac{1}{4}\right), \eta>0$ there exists $\delta_{0}=\delta_{0}\left(C_{1}, C_{2}, \lambda, \varepsilon\right), N_{0} \in \mathbb{N}, N_{0}=N_{0}\left(C_{1}, C_{2}, \lambda, \varepsilon, \delta_{0}\right)$, and $C=C(\eta)>0$ such that for any $\delta \in\left(0, \delta_{0}\right)$, the following holds:

Under the conditions of and with the notation from Proposition 3.12, suppose that for the points $p=\left(x_{p}, y_{p}, z_{p}\right)$ and $q=\left(x_{q}, y_{q}, z_{q}\right)$, the following holds:

1. $0<z_{p}<1$ and $0<z_{q}<1$;

2. For some $N \geq N_{0}$ both $f^{N}(p)$ and $f^{N}(q)$ have z-coordinates larger than 1 , and both $f^{N-1}(p)$ and $f^{N-1}(q)$ have $z$-coordinates not greater than 1 ;

3. There is a smooth curve $\gamma:[0,1] \rightarrow \mathbb{R}^{3}$ such that $\gamma(0)=p, \gamma(1)=q$, and for each $t \in[0,1]$ we have $\gamma^{\prime}(t) \in K_{\gamma(t)}^{u}$; 
If $N \geq N_{0}$ (i.e., if $z_{p}$ is small enough), then

$$
\left|D f^{N}(\mathbf{v})\right| \geq \lambda^{\frac{N}{2}(1-4 \varepsilon)}|\mathbf{v}| \text { for any } \mathbf{v} \in K_{p}^{u},
$$

and if $D f^{N}(\mathbf{v})=\mathbf{u}=\mathbf{u}_{x y}+\mathbf{u}_{z}$, then

$$
\left|\mathbf{u}_{x y}\right|<2 \delta^{1 / 2}\left|\mathbf{u}_{z}\right|
$$

Moreover, if $\left|\mathbf{v}_{z}\right| \geq \eta\left|\mathbf{v}_{x y}\right|$, then

$$
\left|D f^{k}(\mathbf{v})\right| \geq C \lambda^{\frac{k}{2}(1-4 \varepsilon)}|\mathbf{v}| \text { for each } k=1,2, \ldots, N .
$$

In particular,

$$
\operatorname{length}\left(f^{N}(\gamma)\right) \geq \lambda^{\frac{N}{2}(1-4 \varepsilon)} \operatorname{length}(\gamma) .
$$

In order to establish the distortion property we need better control over the expansion rates. In Proposition 3.15 we improve the estimates given by (14) and (17). As a first step we show that, roughly speaking, if a point stays for $N$ iterates in a neighborhood where normalizing coordinates are defined then it must be $\lambda^{-N}$-close to the center-stable manifold of a curve of fixed points.

Proposition 3.14. Given $C_{1}>0, C_{2}>0, \lambda>1$, there exist $\delta_{0}=\delta_{0}\left(C_{1}, C_{2}, \lambda\right), N_{0}=$ $N_{0}\left(C_{1}, C_{2}, \lambda, \delta_{0}\right) \in \mathbb{N}$, and $C^{* *}>C^{*}>0$ such that for any $\delta \in\left(0, \delta_{0}\right)$, the following holds.

Let $f: \mathbb{R}^{3} \rightarrow \mathbb{R}^{3}$ be a $C^{2}$-diffeomorphism such that

(i) $\|f\|_{C^{2}} \leq C_{1}$;

(ii) The planes $\{z=0\}$ and $\{x=0\}$ are invariant under iterates of $f$;

(iii) Every point of the line $\{z=0, x=0\}$ is a fixed point of $f$;

(iv) At a point $Q \in\{z=0, x=0\}$ we have

$$
D f(Q)=\left(\begin{array}{ccc}
\lambda^{-1} & 0 & 0 \\
0 & 1 & 0 \\
0 & 0 & \lambda
\end{array}\right) .
$$

(v) $\|D f(p)-A\|<\delta$ for every $p \in \mathbb{R}^{3}$, where

$$
A=D f(Q)=\left(\begin{array}{ccc}
\lambda^{-1} & 0 & 0 \\
0 & 1 & 0 \\
0 & 0 & \lambda
\end{array}\right) .
$$

Introduce the following cone fields in $\mathbb{R}^{3}$ :

$$
\begin{aligned}
K_{p}^{u} & =\left\{\mathbf{v} \in T_{p} \mathbb{R}^{3}, \mathbf{v}=\mathbf{v}_{x y}+\mathbf{v}_{z}:\left|\mathbf{v}_{z}\right| \geq C_{2} \sqrt{\left|z_{p}\right|}\left|\mathbf{v}_{x y}\right|\right\}, \\
K_{p}^{c u} & =\left\{\mathbf{v} \in T_{p} \mathbb{R}^{3}, \mathbf{v}=\mathbf{v}_{x}+\mathbf{v}_{y z}:\left|\mathbf{v}_{x}\right|<0.01 \lambda^{-1}\left|\mathbf{v}_{y z}\right|\right\}, \\
K_{p}^{s} & =\left\{\mathbf{v} \in T_{p} \mathbb{R}^{3}, \mathbf{v}=\mathbf{v}_{x}+\mathbf{v}_{y z}:\left|\mathbf{v}_{x}\right| \geq C_{2} \sqrt{\left|x_{p}\right|}\left|\mathbf{v}_{y z}\right|\right\}, \\
K_{p}^{c s} & =\left\{\mathbf{v} \in T_{p} \mathbb{R}^{3}, \mathbf{v}=\mathbf{v}_{z}+\mathbf{v}_{x y}:\left|\mathbf{v}_{z}\right|<0.01 \lambda^{-1}\left|\mathbf{v}_{x y}\right|\right\} .
\end{aligned}
$$

Suppose that for a finite orbit $p_{0}, p_{1}, p_{3}, \ldots, p_{N}$ we have

$$
\left(p_{0}\right)_{x} \geq 1,\left(p_{1}\right)_{x}<1,\left(p_{N}\right)_{z} \geq 1,\left(p_{N-1}\right)_{z}<1,
$$


and there are curves $\gamma_{0}$ and $\gamma_{N}$ such that $\gamma_{0}$ connects $p_{0}$ with $W^{s s}(Q)$ and is tangent to both cone fields $K^{u}$ and $K^{c u}$, and $\gamma_{N}$ connects $p_{N}$ with $W^{u u}(Q)$ and is tangent to both cone fields $K^{s}$ and $K^{c s}$.

Then

$$
\begin{aligned}
& C^{*} \lambda^{-N} \leq\left|\left(p_{0}\right)_{z}\right| \leq C^{* *} \lambda^{-N}, \quad \text { and } \\
& C^{*} \lambda^{-N} \leq\left|\left(p_{N}\right)_{x}\right| \leq C^{* *} \lambda^{-N} .
\end{aligned}
$$

Proof. Consider an orthogonal from $p_{0}$ to the plane $\{z=0\}$, and denote its base by $p_{0}^{*}$. There is a unique point $Q_{0}$ on the line $\{z=0, x=0\}$ such that $p_{0}^{*} \in W^{s s}\left(Q_{0}\right)$. Denote the line segment connecting $p_{0}$ and $p_{0}^{*}$ by $\sigma_{0}$ and set $\sigma_{i}=f^{i}\left(\sigma_{0}\right), i=1,2, \ldots, N$.

Similarly, consider an orthogonal from $p_{N}$ to the plane $\{x=0\}$, and denote its base by $p_{N}^{*}$. There is a unique point $Q_{N}$ on the line $\{z=0, x=0\}$ such that $p_{N}^{*} \in W^{u u}\left(Q_{N}\right)$. Denote the line segment connecting $p_{N}$ and $p_{N}^{*}$ by $\rho_{N}$, and set $\rho_{i}=f^{-N+i}\left(\rho_{N}\right), i=$ $0,1,2, \ldots, N-1$.

We have

$$
\begin{array}{ll}
0<\left|\sigma_{0}\right|<\left|\sigma_{1}\right|<\cdots<\left|\sigma_{N-1}\right|<\left|\sigma_{N}\right|, & 1 \leq\left|\sigma_{N}\right| \leq \lambda(1+\delta), \\
0<\left|\rho_{N}\right|<\left|\rho_{N-1}\right|<\cdots<\left|\rho_{1}\right|<\left|\rho_{0}\right|, & 1 \leq\left|\rho_{0}\right| \leq \lambda(1+\delta)
\end{array}
$$

Denote $b_{k}=\operatorname{dist}\left(p_{k}, Q\right)$. Then we have

$$
\begin{aligned}
\left(\lambda^{-1}-\min \left(\delta, C_{1} b_{k}\right)\right)\left|\sigma_{k}\right| & \leq\left|\sigma_{k-1}\right| \leq\left(\lambda^{-1}+\min \left(\delta, C_{1} b_{k}\right)\right)\left|\sigma_{k}\right|, \quad k=1,2, \ldots, N, \\
\left(\lambda^{-1}-\min \left(\delta, C_{1} b_{k}\right)\right)\left|\rho_{k}\right| & \leq\left|\rho_{k+1}\right| \leq\left(\lambda^{-1}+\min \left(\delta, C_{1} b_{k}\right)\right)\left|\rho_{k}\right|, \quad k=0,1,2, \ldots, N-1 .
\end{aligned}
$$

Now we have

$$
\begin{aligned}
b_{k} & =\operatorname{dist}\left(p_{k}, Q\right) \\
& \leq \operatorname{dist}\left(Q, Q_{0}\right)+\left|\rho_{k}\right|+\operatorname{dist}\left(Q, Q_{N}\right)+\left|\sigma_{k}\right| \\
& \leq\left|\sigma_{k}\right|+\left|\rho_{k}\right|+C_{3} C_{2}\left(\sqrt{\left|\rho_{N}\right|}+\sqrt{\left|\sigma_{0}\right|}\right),
\end{aligned}
$$

where $C_{3}$ does not depend on $N$. Indeed, the distance between $p_{0}^{*}$ and $W^{s s}(Q)$ is bounded above by the length of the curve $\gamma_{0}$, and since $\gamma_{0}$ is tangent to the cone fields $K^{u}$ and $K^{c u}$, its length is not greater than $C_{2} \sqrt{\left|\sigma_{0}\right|}$. On the other hand, $\operatorname{dist}\left(Q, Q_{0}\right)$ is of the same order as that distance since the strong stable manifolds of fixed points form a $C^{1}$-foliation of the plane $\{z=0\}$. In the same way one gets an estimate $\operatorname{dist}\left(Q, Q_{N}\right) \leq C_{3} C_{2} \sqrt{\left|\rho_{N}\right|}$.

Since we have the a priori estimates $\left|\sigma_{k}\right| \leq(1+\delta)(\lambda-\delta)^{-N+k}$ and $\left|\rho_{k}\right| \leq(1+\delta)(\lambda-$ $\delta)^{-k}$, we also have

$$
b_{k} \leq\left|\sigma_{k}\right|+\left|\rho_{k}\right|+2 C_{2}(1+\delta)(\lambda-\delta)^{-N / 2} .
$$

If $k<N / 2$, then

$$
\begin{aligned}
b_{k} & \leq(\lambda-\delta)^{-k}\left(1+(\lambda-\delta)^{-N+2 k}+2 C(\lambda-\delta)^{-N / 2+k}\right) \\
& \leq C^{\prime}(\lambda-\delta)^{-k} .
\end{aligned}
$$

If $k \geq N / 2$, then

$$
\begin{aligned}
b_{k} & \leq(\lambda-\delta)^{-N+k}\left(1+(\lambda-\delta)^{N-2 k}+2 C(\lambda-\delta)^{N / 2-k}\right) \\
& \leq C^{\prime}(\lambda-\delta)^{-N+k} .
\end{aligned}
$$


Therefore we have

$$
\begin{aligned}
\left|\sigma_{0}\right| & \leq\left|\sigma_{N}\right| \prod_{k=1}^{N}\left(\lambda^{-1}+\min \left(\delta, C_{1} b_{k}\right)\right) \\
& \leq \lambda(1+\delta) \lambda^{-N} \prod_{k=1}^{[N / 2]}\left(1+C_{1} C^{\prime}(\lambda-\delta)^{-k}\right) \cdot \prod_{k=[N / 2]+1}^{N}\left(1+C_{1} C^{\prime}(\lambda-\delta)^{-N+k}\right) \\
& \leq C^{* *} \lambda^{-N} .
\end{aligned}
$$

Also,

$$
\begin{aligned}
\left|\sigma_{0}\right| & \geq\left|\sigma_{N}\right| \prod_{k=1}^{N}\left(\lambda^{-1}-\min \left(\delta, C_{1} b_{k}\right)\right) \\
& \geq \lambda^{-N} \prod_{k=1}^{[N / 2]}\left(1-C_{1} C^{\prime}(\lambda-\delta)^{-k}\right) \cdot \prod_{k=[N / 2]+1}^{N}\left(1-C_{1} C^{\prime}(\lambda-\delta)^{-N+k}\right) \\
& \geq C^{*} \lambda^{-N}
\end{aligned}
$$

In the same way we get estimates for $\rho_{N}$.

Proposition 3.15. Given $C_{1}>0, C_{2} \geq 0, \lambda>1$, there exist $\delta_{0}=\delta_{0}\left(C_{1}, C_{2}, \lambda\right), N_{0} \in$ $\mathbb{N}, N_{0}=N_{0}\left(C_{1}, C_{2}, \lambda, \delta_{0}\right) \in \mathbb{N}$, and $\widetilde{C}>0$ such that for any $\delta \in\left(0, \delta_{0}\right)$, the following holds:

Under the conditions of and with the notation from Proposition 3.14, suppose that $\mathbf{v} \in T_{p_{0}} \mathbb{R}^{3}, \mathbf{v} \in K_{p_{0}}^{u}$. Then $\left|D f_{p_{0}}^{N}(\mathbf{v})\right| \geq \widetilde{C} \lambda^{N / 2}|\mathbf{v}|$.

Proof. We will use the notation from Proposition 3.14 and its proof.

Let us denote $\mathbf{v}_{k}=D f^{k}(\mathbf{v}), k=0,1, \ldots, N$, and $D_{k}=\left|\left(\mathbf{v}_{k}\right)_{z}\right|, d_{k}=\left|\left(\mathbf{v}_{k}\right)_{x y}\right|$. Let us normalize $\mathbf{v}$ in such a way that $d_{0}=1$. Since $\mathbf{v} \in K_{p_{0}}^{u}$ and $\left|\sigma_{0}\right| \geq C^{*} \lambda^{-N}$, we have $D_{0} \geq C_{5} \lambda^{-N / 2}$, where $C_{5}$ is independent of $N$.

Denote

$$
D f(p)=\left(\begin{array}{lll}
v(p) & m_{1}(p) & t_{1}(p) \\
m_{2}(p) & e(p) & t_{2}(p) \\
s_{1}(p) & s_{2}(p) & \lambda(p)
\end{array}\right)
$$

We have

$$
D f(p)(\mathbf{v})=\left(\begin{array}{lcc}
v(p) & m_{1}(p) & t_{1}(p) \\
m_{2}(p) & e(p) & t_{2}(p) \\
s_{1}(p) & s_{2}(p) & \lambda(p)
\end{array}\right)\left(\begin{array}{l}
\mathbf{v}_{x} \\
\mathbf{v}_{y} \\
\mathbf{v}_{z}
\end{array}\right)=\left(\begin{array}{l}
v(p) \mathbf{v}_{x}+m_{1}(p) \mathbf{v}_{y}+t_{1}(p) \mathbf{v}_{z} \\
m_{2}(p) \mathbf{v}_{x}+e(p) \mathbf{v}_{y}+t_{2}(p) \mathbf{v}_{z} \\
s_{1}(p) \mathbf{v}_{x}+s_{2}(p) \mathbf{v}_{y}+\lambda(p) \mathbf{v}_{z}
\end{array}\right) .
$$

Since $\|f\|_{C^{2}} \leq C_{1}$, we also have $|v(p)| \leq \lambda^{-1}+C_{1} \operatorname{dist}(Q, p),\left|m_{1}(p)\right|,\left|m_{2}(p)\right|,\left|t_{1}(p)\right|$, $\left|t_{2}(p)\right| \leq C_{1} \operatorname{dist}(Q, p)$, and $|\lambda(p)| \geq \lambda-C_{1} \operatorname{dist}(Q, p)$. Furthermore, if $p$ belongs to the plane $\{z=0\}$, then $s_{1}(p)=s_{2}(p)=0$. Therefore, for arbitrary $p$, we have $\left|s_{1}(p)\right|,\left|s_{2}(p)\right| \leq C_{1} z_{p}$. This implies that we have the following estimates:

$$
\left\{\begin{array}{l}
d_{k+1} \leq\left(1+\min \left(\delta, C_{1} b_{k}\right)\right) d_{k}+\min \left(\delta, C_{1} b_{k}\right) D_{k} \\
D_{k+1} \geq\left(\lambda-\min \left(\delta, C_{1} b_{k}\right)\right) D_{k}-\min \left(\delta, C_{1}\left|\sigma_{k}\right|\right) d_{k}
\end{array} .\right.
$$


Lemma 3.16. There exists $k^{*}$ such that $d_{k} \geq D_{k}$ for all $k \leq k^{*}$, and $d_{k}<D_{k}$ for all $k>k^{*}$.

Proof. Indeed, if $D_{k}>d_{k}$, then

$$
d_{k+1} \leq(1+\delta) d_{k}+\delta D_{k} \leq(1+2 \delta) D_{k}
$$

and

$$
D_{k+1} \geq(\lambda-\delta) D_{k}-\delta d_{k} \geq(\lambda-2 \delta) D_{k}
$$

Since $\lambda-2 \delta>1+2 \delta$, we have $D_{k+1}>d_{k+1}$.

We have the following preliminary estimates. If $k<N / 2$, then $b_{k} \leq C^{\prime}(\lambda-\delta)^{-k}$; if $k \geq N / 2$, then $b_{k} \leq C^{\prime}(\lambda-\delta)^{-N+k}$. Also, $\left|\sigma_{k}\right| \leq(\lambda-\delta)^{-N+k}$ for each $k=0,1, \ldots, N$. Notice that this implies that $\prod_{i=1}^{N}\left(1+C_{1} b_{i}\right)$ is bounded by a constant that is independent of $N$. And, finally, $D_{k} \geq C_{5} \lambda^{-N / 2}(\lambda-\delta)^{k}$; see [DG09a, Lemma 6.1].

If $D_{k} \leq d_{k}$ (i.e., $k \leq k^{*}$ ), then

$$
\begin{aligned}
d_{k+1} & \leq\left(1+C_{1} b_{k}\right) d_{k}+C_{1} b_{k} D_{k} \\
& \leq\left(1+2 C_{1} b_{k}\right) d_{k} \\
& \leq\left[\prod_{i=1}^{k}\left(1+2 C_{1} b_{k}\right)\right] d_{0} \\
& \leq C_{6},
\end{aligned}
$$

where $C_{6}$ does not depend on $k$ or $N$. Moreover, we have

$$
\begin{aligned}
D_{k+1} & \geq\left(\lambda-C_{1} b_{k}\right) D_{k}-C_{1}\left|\sigma_{k}\right| d_{k} \\
& \geq\left(\lambda-C_{1} b_{k}\right) D_{k}-C_{1} C_{6}(\lambda-\delta)^{-N+k} \\
& \geq\left(\lambda-C_{1} b_{k}\right) D_{k}\left(1-\frac{C_{1} C_{6}}{\lambda-C_{1} b_{k}} \cdot \frac{(\lambda-\delta)^{-N+k}}{D_{k}}\right) \\
& \geq\left(\lambda-C_{1} b_{k}\right) D_{k}\left(1-\frac{C_{1} C_{6}}{\lambda-C_{1} b_{k}} \cdot \frac{(\lambda-\delta)^{-N+k}}{C_{5} \lambda^{-N / 2}(\lambda-\delta)^{k}}\right) \\
& \geq\left(\lambda-C_{1} b_{k}\right) D_{k}\left(1-\left(\frac{C_{1} C_{6}}{C_{5}\left(\lambda-C_{1} b_{k}\right)}\right)\left(\lambda^{1 / 2}(\lambda-\delta)^{-1}\right)^{N}\right) \\
& \geq \lambda^{k+1} D_{0}\left[\prod_{i=0}^{k}\left(1-\left(C_{1} \lambda^{-1}\right) b_{i}\right)\right] \cdot\left(1-\left(\frac{C_{1} C_{6}}{C_{5}\left(\lambda-C_{1} b_{k}\right)}\right)\left(\lambda^{1 / 2}(\lambda-\delta)^{-1}\right)^{N}\right)^{k} \\
& \geq \lambda^{k+1} D_{0}\left[\prod_{i=0}^{k}\left(1-\left(C_{1} \lambda^{-1}\right) b_{i}\right)\right] \cdot\left(1-\left(\frac{C_{1} C_{6}}{C_{5}\left(\lambda-C_{1} b_{k}\right)}\right)\left(\lambda^{1 / 2}(\lambda-\delta)^{-1}\right)^{N}\right)^{N} \\
& \geq C_{7} \lambda^{k+1} D_{0},
\end{aligned}
$$

since for any $C>0$ and $\xi \in(0,1)$, one has $\lim _{N \rightarrow \infty}\left(1-C \xi^{N}\right)^{N}=1$. 
If $d_{k}<D_{k}$ (i.e., $k>k^{*}$ ), then

$$
\begin{aligned}
D_{k+1} & \geq\left(\lambda-C_{1} b_{k}\right) D_{k}-C_{1}\left|\sigma_{k}\right| d_{k} \\
& \geq\left(\lambda-C_{1} b_{k}-C_{1}\left|\sigma_{k}\right|\right) D_{k} \\
& \geq \lambda D_{k}\left(1-\lambda^{-1} C_{1} b_{k}-\lambda^{-1} C_{1}\left|\sigma_{k}\right|\right) \\
& \geq \lambda^{k+1} C_{7} D_{0} \prod_{i=k^{*}}^{k}\left(1-\lambda^{-1} C_{1} b_{k}-\lambda^{-1} C_{1}\left|\sigma_{k}\right|\right) \\
& \geq C_{8} \lambda^{k+1} D_{0},
\end{aligned}
$$

where $C_{8}$ does not depend on $N$ or $k$.

Finally, $\left|D f_{p_{0}}^{N}(\mathbf{v})\right| \geq D_{N} \geq C_{8} \lambda^{N} D_{0} \geq \widetilde{C} \lambda^{N / 2}|\mathbf{v}|$.

Below (in the proof of Proposition 3.18) we will also need an estimate on $k^{*}$ provided by Lemma 3.16. Namely, we claim that $k^{*}$ cannot be much larger than $N / 2$. The formal statement is the following.

Lemma 3.17. There is a constant $C_{9}$ independent of $N$ such that

$$
\lambda^{k^{*}} \leq C_{9} \lambda^{N / 2}
$$

Proof. We know that $D_{k^{*}-1} \leq d_{k^{*}-1} \leq C_{6}$. Therefore $C_{6} \geq D_{k^{*}-1} \geq C_{7} \lambda^{k^{*}} D_{0} \geq$ $C_{7} \lambda^{k^{*}} \cdot C_{5} \lambda^{-N / 2}$, so $\lambda^{k^{*}} \leq\left(C_{6} C_{7}^{-1} C_{5}^{-1}\right) \lambda^{N / 2}$.

Now we are ready to formulate the statement that will be used to check the distortion property of the trace map.

Proposition 3.18. Given $C_{1}>0, C_{2}>0, C_{3}>0, \lambda>1$, there exist $\delta_{0}=$ $\delta_{0}\left(C_{1}, C_{2}, C_{3}, \lambda\right), N_{0}=N_{0}\left(C_{1}, C_{2}, C_{3}, \lambda, \delta_{0}\right) \in \mathbb{N}$, and $C>0$ such that for any $\delta \in\left(0, \delta_{0}\right)$ and any $\Delta>0$, the following holds:

Under the conditions of and with the notation from Proposition 3.14, suppose that the curve $\gamma_{0}$ has a curvature bounded by $C_{3}$. Suppose also that for the points $p=$ $\left(x_{p}, y_{p}, z_{p}\right)$ and $q=\left(x_{q}, y_{q}, z_{q}\right)$, the following holds:

1. $p, q \in \gamma_{0}$;

2. For some $N \geq N_{0}$ both $f^{N}(p)$ and $f^{N}(q)$ have $z$-coordinates larger than 1 , and both $f^{N-1}(p)$ and $f^{N-1}(q)$ have $z$-coordinates not greater than 1;

3. $\operatorname{dist}\left(f^{N}(p), f^{N}(q)\right)=\Delta$.

Denote $p_{k}=f^{k}(p), q_{k}=f^{k}(q), k=0, \ldots, N$.

Let $\mathbf{v} \in T_{p} \mathbb{R}^{3}$ and $\mathbf{w} \in T_{q} \mathbb{R}^{3}$ be vectors tangent to $\gamma_{0}$.

Denote $\mathbf{v}_{k}=D f^{k}(v)$ and $\mathbf{w}_{k}=D f^{k}(w), k=0, \ldots, N$. Let $\alpha_{k}$ be the angle between $\mathbf{v}_{k}$ and $\mathbf{w}_{k}$.

Then

$$
\sum_{k=0}^{N} \alpha_{k}<C \Delta, \text { and } \sum_{k=0}^{N} \operatorname{dist}\left(p_{k}, q_{k}\right)<C \Delta .
$$


Proof. First of all, notice that it is enough to prove Proposition 3.18 in the case when the points $p$ and $q$ are arbitrarily close to each other. Indeed, otherwise split the piece of the curve $\gamma_{0}$ between the points $p$ and $q$ into a large number of extremely small pieces. If for each of them the statement of Proposition 3.18 holds, then by subadditivity of the inequalities (23) it holds in general.

Denote by $\Gamma$ the piece of the curve $\gamma_{0}$ between the points $p_{0}$ and $q_{0}$, and set $\Gamma_{k}=$ $f^{k}(\Gamma), k=0,1,2, \ldots, N$. Denote $\mu_{k}=\left|\Gamma_{k}\right|$. Due to the remark above we can assume that for any vector tangent to $\Gamma$, the value of $k^{*}$ is the same. From the proof of Proposition 3.15 we see that for $k=0,1, \ldots, k^{*}$, we have $\mu_{k} \leq C_{6} \mu_{0}$, and $\Delta \approx \mu_{N} \geq$ $\widetilde{C} \lambda^{N / 2} \mu_{0} \geq \widetilde{C} \lambda^{N / 2} C_{6}^{-1} \mu_{k^{*}}$, so $\mu_{k^{*}} \leq\left(\widetilde{C}^{-1} C_{6}\right) \lambda^{-N / 2} \mu_{N} \leq C_{11} \lambda^{-N / 2} \Delta$.

On the other hand, if $k>k^{*}$, then

$$
\Delta \approx \mu_{N} \geq D_{N} \geq C_{7} \lambda^{N-k} D_{k^{*}} \geq C_{7} \lambda^{N-k} \frac{1}{2} \mu_{k},
$$

where we denote by $D_{k}$ the length of the projection of $\Gamma_{k}$ to the $z$-axis (slightly abusing the notation). Therefore, $\mu_{k} \leq\left(2 C_{7}^{-1}\right) \lambda^{-N+k} \Delta$.

It follows that we have

$$
\begin{aligned}
\sum_{k=0}^{N} \operatorname{dist}\left(p_{k}, q_{k}\right) & \leq \sum_{k=0}^{N} \mu_{k} \\
& =\sum_{k=0}^{k^{*}} \mu_{k}+\sum_{k=k *+1}^{N} \mu_{k} \\
& \leq k^{*} \cdot C_{6} \mu_{0}+\sum_{k=k^{*}+1}^{N}\left(2 C_{7}^{-1}\right) \lambda^{-N+k} \Delta \\
& \leq k^{*} \cdot \widetilde{C}^{-1} \lambda^{-N / 2} C_{6} \Delta+C_{12} \Delta \\
& \leq C \Delta .
\end{aligned}
$$

Notice that for any two vectors $\mathbf{v}, \mathbf{w} \in K^{c u}$,

$$
\angle(A \mathbf{v}, A \mathbf{w}) \leq \lambda \angle(\mathbf{v}, \mathbf{w}), \quad \text { where } A=\left(\begin{array}{ccc}
\lambda^{-1} & 0 & 0 \\
0 & 1 & 0 \\
0 & 0 & \lambda
\end{array}\right),
$$

and if a linear operator $B$ is $\xi$-close to $A$, then

$$
\begin{aligned}
\angle(B \mathbf{v}, B \mathbf{w}) & \leq(\lambda+\xi)(1+\xi) \angle(\mathbf{v}, \mathbf{w}) \\
& =(\lambda+\xi(\lambda+1+\xi)) \angle(\mathbf{v}, \mathbf{w}) \\
& <\lambda(1+2 \xi) \angle(\mathbf{v}, \mathbf{w}) .
\end{aligned}
$$

Therefore we have

$$
\alpha_{0} \leq C_{3} \mu_{0}
$$

and

$$
\alpha_{k+1} \leq \lambda\left(1+2 C_{1} b_{k}\right) \alpha_{k}+C_{1} \mu_{k}, \quad k=0,1, \ldots, k^{*}
$$


Since $\prod_{k=0}^{k^{*}}\left(1+2 C_{1} b_{k}\right) \leq C_{13}$ for some $C_{13}$ that is independent of $k^{*}$ and $N$, we have

$$
\alpha_{k} \leq\left(\lambda^{k}+\lambda^{k-1}+\cdots+\lambda+1\right) \cdot\left(C_{13} C_{1} C_{6} C_{3}\right) \mu_{0} \leq C_{14} \lambda^{k} \mu_{0},
$$

where $C_{14}$ is also independent of $k$ and $N$. In particular,

$$
\alpha_{k^{*}} \leq C_{14} \lambda^{k^{*}} \mu_{0} \leq\left(C_{14} C_{9}\right) \lambda^{N / 2} \widetilde{C}^{-1} \lambda^{-N / 2} \Delta \leq C_{15} \Delta
$$

and

$$
\sum_{k=0}^{k^{*}} \alpha_{k} \leq \sum_{k=0}^{k^{*}} C_{14} \lambda^{k} \mu_{0} \leq C_{16} \lambda^{k^{*}} \mu_{0} \leq C_{17} \Delta
$$

Now denote

$$
K_{p}^{u u}=\left\{\mathbf{v} \in T_{p} \mathbb{R}^{3}:\left|\mathbf{v}_{z}\right|>100 \lambda\left|\mathbf{v}_{x y}\right|\right\}
$$

If $\mathbf{v}, \mathbf{w} \in K^{u u}$, then $\angle(A \mathbf{v}, A \mathbf{w}) \leq \lambda^{-1 / 2} \angle(\mathbf{v}, \mathbf{w})$, and the same holds for any linear operator $B$ which is $\delta$-close to $A$.

There exists $m \in \mathbb{N}$ independent of $N$ such that if for a vector $\mathbf{v}$ we have $\left|\mathbf{v}_{z}\right|>\left|\mathbf{v}_{x y}\right|$, then $D f^{m}(\mathbf{v}) \in K^{u u}$.

Also

$$
\alpha_{k^{*}+m}=\angle\left(D f^{m}\left(v_{k^{*}}\right), D f^{m}\left(w_{k^{*}}\right)\right) \leq C_{15}(\lambda+2 \delta)^{m} \Delta=C_{16} \Delta,
$$

and for $k \geq k^{*}+m$, we have $\alpha_{k+1} \leq \lambda^{-1 / 2} \alpha_{k}+C_{1} \mu_{k}$.

Denote $v=\lambda^{-1 / 3}-\lambda^{-1 / 2}$.

If $C_{1} \mu_{k}<v \alpha_{k}$, then

$$
\alpha_{k+1} \leq \lambda^{-1 / 2} \alpha_{k}+v \alpha_{k}=\left(\lambda^{-1 / 2}+v\right) \alpha_{k}=\lambda^{-1 / 3} \alpha_{k}
$$

If $C_{1} \mu_{k} \geq v \alpha_{k}$, then

$$
\alpha_{k+1} \leq \lambda^{-1 / 2} \alpha_{k}+C \mu_{k} \leq\left(\lambda^{-1 / 2} v^{-1}+1\right) C_{1} \mu_{k} .
$$

Since $\sum_{k=k^{*}+m}^{N} \mu_{k} \leq \sum_{k=k^{*}+m}^{N}\left(2 C_{7}^{-1}\right) \lambda^{-N+k} \Delta \leq C_{12} \Delta$, this implies that

$$
\sum_{k=k^{*}+m}^{N} \alpha_{k} \leq C_{18} \Delta
$$

and

$$
\sum_{k=0}^{N} \alpha_{k} \leq \sum_{k=0}^{k^{*}} \alpha_{k}+\left(\alpha_{k^{*}+1}+\cdots+\alpha_{k^{*}+m-1}\right)+\sum_{k=k^{*}+m}^{N} \alpha_{k} \leq C \Delta
$$

concluding the proof. 
3.6. Distortion property: preliminary estimates. The main result of this subsection is the following statement:

Lemma 3.19. There are constants $R>0, V_{0}>0$, and $\kappa>0$ such that for any $V \in$ $\left(0, V_{0}\right)$ and $N \in \mathbb{N}$, the following holds: Suppose that $\gamma \subset T^{N}\left(\mathbf{l}_{V}\right) \backslash O_{r_{1}}$ is a connected curve of length not greater than $\kappa$. Let the points $p, q \in \mathbf{l}_{V}$ be such that $T_{V}^{N}(p) \in \gamma$ and $T_{V}^{N}(q) \in \gamma$, and $v_{p}$ and $v_{q}$ be unit vectors tangent to $\gamma$ at points $p$ and $q$. Then

$$
\sum_{i=0}^{N}\left(\angle\left(D T_{V}^{i}\left(v_{p}\right), D T_{V}^{i}\left(v_{q}\right)\right)+\operatorname{dist}\left(T_{V}^{i}(p), T_{V}^{i}(q)\right)\right)<R .
$$

Notice that $F^{-1}\left(\mathbb{S} \backslash O_{r_{2}}\right)$ is a torus without small neighborhoods of the preimages of the singularities, and we can define the following map:

$$
\widetilde{T}_{V}: F^{-1}\left(\mathbb{S} \backslash O_{r_{2}}\right) \rightarrow \mathbb{T}^{2}, \quad \widetilde{T}_{V}=F^{-1} \circ \pi_{V} \circ T_{V} \circ \pi_{V}^{-1} \circ F \equiv F_{V}^{-1} \circ T_{V} \circ F_{V} .
$$

If $V$ is small, $\widetilde{T}_{V}$ is $C^{2}$-close to the linear automorphism $\mathcal{A}$ on its domain.

Lemma 3.20. For $V_{0}>0$ small enough, there exists $t \in(0,1)$ such that for $V \in$ $\left[0, V_{0}\right], p, q \in \mathbb{T}^{2} \backslash F^{-1}\left(O_{r_{2}}\right)$ and unit vectors $v_{p} \in K_{p}^{u}, v_{q} \in K_{q}^{u}$, we have

$$
\angle\left(D \widetilde{T}_{V, p}\left(v_{p}\right), D \widetilde{T}_{V, q}\left(v_{q}\right)\right) \leq t \angle\left(v_{p}, v_{q}\right)+2\left\|\widetilde{T}_{V}\right\|_{C^{2}} \operatorname{dist}(p, q) .
$$

Proof. If $V_{0}$ is small, then $\widetilde{T}_{V}$ is $C^{2}$-close to the linear automorphism $\mathcal{A}$. In particular, for any point $p \in \mathbb{T}^{2} \backslash F^{-1}\left(O_{r_{2}}\right)$ and any vectors $v_{1}, v_{2} \in K_{p}^{u}$,

$$
\angle\left(D \widetilde{T}_{V, p}\left(v_{1}\right), D \widetilde{T}_{V, p}\left(v_{2}\right)\right) \leq t \angle\left(v_{1}, v_{2}\right),
$$

where $t \in(0,1)$ can be chosen uniformly for all $V \in\left[0, V_{0}\right]$ and $p \in \mathbb{T}^{2} \backslash F^{-1}\left(O_{r_{2}}\right)$. Therefore we have

$$
\begin{aligned}
\angle\left(D \widetilde{T}_{V, p}\left(v_{p}\right), D \widetilde{T}_{V, q}\left(v_{q}\right)\right) & \leq \angle\left(D \widetilde{T}_{V, p}\left(v_{p}\right), D \widetilde{T}_{V, p}\left(v_{q}\right)\right)+\angle\left(D \widetilde{T}_{V, p}\left(v_{q}\right), D \widetilde{T}_{V, q}\left(v_{q}\right)\right) \\
& \leq t \angle\left(v_{p}, v_{q}\right)+2\left\|D \widetilde{T}_{V, p}\left(v_{q}\right)-D \widetilde{T}_{V, q}\left(v_{q}\right)\right\| \\
& \leq t \angle\left(v_{p}, v_{q}\right)+2\left\|\widetilde{T}_{V}\right\|_{C^{2}} \operatorname{dist}(p, q),
\end{aligned}
$$

as claimed.

Definition 3.21. For any points $p, q$ and any vectors $v_{p}, v_{q}$ define

$$
\mathfrak{F}\left(p, q, v_{p}, v_{q}\right) \equiv \frac{\angle\left(v_{p}, v_{q}\right)}{\operatorname{dist}(p, q)} .
$$

Lemma 3.22. For $p, q \in \mathbb{T}^{2} \backslash F^{-1}\left(O_{r_{2}}\right), p \neq q$, and vectors $v_{p} \in K_{p}^{u}, v_{q} \in K_{q}^{u}$, consider the function $\mathfrak{F}\left(p, q, v_{p}, v_{q}\right)$ defined by (25). Suppose that $p$ and $q$ belong to $a$ curve that is tangent to the unstable cone field. Then

$$
\mathfrak{F}\left(\widetilde{T}_{V}(p), \widetilde{T}_{V}(q), D \widetilde{T}_{V, p}\left(v_{p}\right), D \widetilde{T}_{V, q}\left(v_{q}\right)\right) \leq t \mathfrak{F}\left(p, q, v_{p}, v_{q}\right)+2\left\|\widetilde{T}_{V}\right\|_{C^{2}} .
$$

In particular, if $\mathfrak{F}\left(p, q, v_{p}, v_{q}\right)>\frac{4\left\|\widetilde{T}_{V}\right\|_{C^{2}}}{1-t}$, then

$$
\mathfrak{F}\left(\widetilde{T}_{V}(p), \widetilde{T}_{V}(q), D \widetilde{T}_{V, p}\left(v_{p}\right), D \widetilde{T}_{V, q}\left(v_{q}\right)\right) \leq \frac{1+t}{2} \mathfrak{F}\left(p, q, v_{p}, v_{q}\right) \text {. }
$$


Proof. We have

$$
\begin{aligned}
\mathfrak{F}\left(\widetilde{T}_{V}(p), \widetilde{T}_{V}(q), D \widetilde{T}_{V, p}\left(v_{p}\right), D \widetilde{T}_{V, q}\left(v_{q}\right)\right) & =\frac{\angle\left(D \widetilde{T}_{V, p}\left(v_{p}\right), D \widetilde{T}_{V, q}\left(v_{q}\right)\right)}{\operatorname{dist}\left(\widetilde{T}_{V}(p), \widetilde{T}_{V}(q)\right)} \\
& \leq \frac{t \angle\left(v_{p}, v_{q}\right)+2\left\|\widetilde{T}_{V}\right\|_{C^{2}} \operatorname{dist}(p, q)}{\operatorname{dist}(p, q)} \\
& =t \mathfrak{F}\left(p, q, v_{p}, v_{q}\right)+2\left\|\widetilde{T}_{V}\right\|_{C^{2}} .
\end{aligned}
$$

If we also have $\mathfrak{F}\left(p, q, v_{p}, v_{q}\right)>\frac{4\left\|\widetilde{T}_{V}\right\|_{C^{2}}}{1-t}$, then

$$
\begin{aligned}
t \mathfrak{F}\left(p, q, v_{p}, v_{q}\right)+2\left\|\widetilde{T}_{V}\right\|_{C^{2}} & \leq t \mathfrak{F}\left(p, q, v_{p}, v_{q}\right)+\frac{1-t}{2} \mathfrak{F}\left(p, q, v_{p}, v_{q}\right) \\
& =\frac{1+t}{2} \mathfrak{F}\left(p, q, v_{p}, v_{q}\right) .
\end{aligned}
$$

Lemma 3.5 immediately implies the following statement.

Lemma 3.23. Fix a small $V \geq 0$. Suppose that $a, b \in \mathbb{T}^{2} \backslash F^{-1}\left(O_{r_{2}}\right)$ are such that $\Psi_{V}(a)$ and $\Psi_{V}(b)$ are defined, and $v_{a} \in T_{a} \mathbb{T}^{2} \backslash F^{-1}\left(O_{r_{2}}\right), v_{b} \in T_{b} \mathbb{T}^{2} \backslash F^{-1}\left(O_{r_{2}}\right)$. Then

$$
\mathfrak{F}\left(a, b, v_{a}, v_{b}\right) \leq \widetilde{C}^{2}\left(\mathfrak{F}\left(\Psi_{V}(a), \Psi_{V}(b), D \Psi_{V}\left(v_{a}\right), D \Psi_{V}\left(v_{b}\right)\right)+1\right)
$$

and

$$
\mathfrak{F}\left(\Psi_{V}(a), \Psi_{V}(b), D \Psi_{V}\left(v_{a}\right), D \Psi_{V}\left(v_{b}\right)\right) \leq \widetilde{C}^{2}\left(\mathfrak{F}\left(a, b, v_{a}, v_{b}\right)+1\right) .
$$

Since $\widetilde{T}_{V}$ is $C^{2}$-close to the linear automorphism $\mathcal{A}$ on its domain for small $V \geq 0$, we can assume that for all $V \in\left[0, V_{0}\right]$, we have $\left\|\widetilde{T}_{V}\right\|_{C^{2}} \leq 10$.

Let $C$ be the constant from Proposition 3.18, where $C_{3}=\left(\frac{40}{1-t}+1\right) \widetilde{C}^{2}$ was taken. Fix a small $\tau>0$. Take $n^{*} \in \mathbb{N}$ such that

$$
\left(\frac{1+t}{2}\right)^{n^{*}} \widetilde{C}^{2}(C+1) \leq \frac{40}{1-t}, \quad \text { and } \quad \widetilde{C}^{2} \mu^{n^{*}} \geq \mu^{n^{*}\left(1-\frac{\tau}{8}\right)} .
$$

Now we are going to choose a neighborhood $U$ of the set of singularities $\left\{P_{1}, P_{2}, P_{3}, P_{4}\right\}$ in such a way that if an orbit of a point leaves $U$, then it does not enter $U$ for the next $n^{*}$ iterates. Also, we will choose a smaller neighborhood $U^{*} \subset U$ such that if a small curve is tangent to an unstable cone field (see Definition 3.8) and intersects $U^{*}$, then either it is entirely inside of $U$, or its iterates will continue to intersect $U$ until they reach the opposite rectangle. Here is how we do that.

For small $r_{3} \in\left(0, r_{2}\right)$, we denote $O_{r_{3}}^{0}=O_{r_{3}}, O_{r_{3}}^{1}=T\left(O_{r_{3}}\right) \cap O_{r_{1}}, O_{r_{3}}^{i}=T\left(O_{r_{3}}^{i-1}\right) \cap$ $O_{r_{1}}$ for each $i>1, O_{r_{3}}^{i}=T^{-1}\left(O_{r_{3}}^{i+1}\right) \cap O_{r_{1}}$ for each $i<0$, and $U=\cup_{i=-\infty}^{\infty}\left(O_{r_{3}}^{i}\right)$. We will take $r_{3}$ so small that the following property holds. If $p \in S_{V}$ is such that $T^{-1}(p) \in U$ but $p \notin U$, then $T^{n}(p) \notin U$ for every $n \in \mathbb{N}$ with $n \leq n^{*}$.

For small $r_{4} \in\left(0, r_{3}\right)$, we denote $O_{r_{4}}^{0}=O_{r_{4}}, O_{r_{4}}^{1}=T\left(O_{r_{4}}\right) \cap O_{r_{1}}, O_{r_{4}}^{i}=T\left(O_{r_{4}}^{i-1}\right) \cap$ $O_{r_{1}}$ for each $i>1, O_{r_{4}}^{i}=T^{-1}\left(O_{r_{4}}^{i+1}\right) \cap O_{r_{1}}$ for each $i<0$, and

$$
U^{*}=\bigcup_{i=-\infty}^{\infty}\left(O_{r_{4}}^{i}\right)
$$


We will take $r_{4}$ so small that the following property holds. Suppose $\gamma$ is a curve on $\mathbb{T}^{2}$ such that $\Psi_{V}(\gamma)$ is defined, $F_{V}(\gamma) \subset O_{r_{1}}, \gamma$ is tangent to the unstable cone field, $F_{V}(\gamma) \cap O_{r_{1}} \backslash U \neq \emptyset$, and $F_{V}(\gamma) \cap U^{*} \neq \emptyset$. Then there is $k \in \mathbb{N}$ such that $T_{V}^{n}\left(F_{V}(\gamma)\right) \cap$ $U \neq \varnothing$ for all natural $n \leq k$, and $T_{V}^{k}\left(F_{V}(\gamma)\right)$ intersects the opposite rectangle of the Markov partition.

Lemma 3.24. There are $V_{0}>0$ and $R_{1}>0$ such that the following holds. Suppose that $v$ is a non-zero vector tangent to the line $\mathbf{l}_{V}$ at some point $p \in \mathbf{l}_{V}$. Let $N \in \mathbb{N}$ be such that $T_{V}(p)$ belongs to the bounded component of $S_{V} \backslash O_{r_{1}}$. Then

$$
\sum_{i=0}^{N}\left\|D T_{V}^{i}(v)\right\| \leq R_{1}\left\|D T_{V}^{N}(v)\right\| .
$$

Proof. If $V$ is small enough, the vector $D T^{m_{0}}(v)$ is tangent to the unstable cone field. Let us split the orbit $\left\{T^{m_{0}}(p), T^{m_{0}+1}(p), \ldots, T^{N}(p)\right\}$ into several intervals

$$
\left\{T^{m_{0}}(q), T^{m_{0}+1}(q), \ldots, T^{k_{1}-1}(q)\right\},\left\{T^{k_{1}}(q), \ldots, T^{k_{2}-1}(q)\right\}, \ldots,\left\{T^{k_{s}}(q), \ldots, T^{N}(q)\right\}
$$

in such a way that the following properties hold:

(1) for each $i=1,2, \ldots, s$, the points $T^{k_{i}-1}(q)$ and $T^{k_{i}}(q)$ are outside of $O_{r_{2}}$;

(2) if $\left\{T^{k_{i}}(q), \ldots, T^{k_{i+1}-1}(q)\right\} \cap U^{*} \neq \varnothing$, then $\left\{T^{k_{i}}(q), \ldots, T^{k_{i+1}-1}(q)\right\} \subset U^{*}$;

(3) for each $i=1,2, \ldots, s-1$, we have either $k_{i+1}-k_{i} \geq n^{*}$ (where $n^{*}$ is chosen due to (26)) or $\left\{T^{k_{i}}(q), \ldots, T^{k_{i+1}-1}(q)\right\} \cap U^{*} \neq \emptyset$.

Such a splitting exists due to the choice of $U^{*} \subset U$ in (27) above.

Apply Proposition 3.18 to those intervals in the splitting that are contained in $U^{*}$. The choice of $n^{*}$ in (26) above guarantees for the intervals that do not intersect $U^{*}$ uniform expansion of the vector. The first and the last interval may have length greater than $n^{*}$, and then we have uniform expansion that "kills" the distortion added by the change of coordinates, or smaller than $n^{*}$, but then they do not add more than a constant to the sum. As a result, the required sum is bounded above by a geometrical progression.

Lemma 3.24 implies the following statement.

Lemma 3.25. There are constants $R_{1}>0, V_{0}>0$, and $\kappa_{1}>0$ such that for any $V \in\left(0, V_{0}\right)$ and $N \in \mathbb{N}$, the following holds: Suppose that $\gamma \subset T^{N}\left(\mathbf{l}_{V}\right) \backslash O_{r_{1}}$ is a connected curve of length not greater than $\kappa_{1}$. Let the points $p, q \in \mathbf{l}_{V}$ be such that $T_{V}^{N}(p) \in \gamma$ and $T_{V}^{N}(q) \in \gamma$. Then

$$
\sum_{i=0}^{N} \operatorname{dist}\left(T_{V}^{i}(p), T_{V}^{i}(q)\right)<R_{1} .
$$

Finally, the choice of $n^{*}$ and Proposition 3.18 imply that the function $\mathfrak{F}\left(T_{V}^{i}(p), T_{V}^{i}(q)\right.$, $\left.D T_{V}^{i}\left(v_{p}\right), D T_{V}^{i}\left(v_{q}\right)\right)$ is uniformly bounded, and together with Lemma 3.25 this proves Lemma 3.19. 


\subsection{Proof of the distortion property.}

Proof of Proposition 3.11. Notice that we need to prove that

$$
\left|\log \left(\frac{\left|\Gamma_{G}\right|\left|\gamma_{B}\right|}{\left|\Gamma_{B}\right|\left|\gamma_{G}\right|}\right)\right|
$$

is bounded by some constant independent of the choice of the gap and of $V$. There are points $p_{G} \in \gamma_{G}$ and $p_{B} \in \gamma_{B}$ such that if $v_{G}$ is a unit vector tangent to the curve $\gamma_{G}$ at $p_{G}$, and $v_{B}$ is a unit vector tangent to the curve $\gamma_{B}$ at $p_{B}$, then

$$
\begin{aligned}
\left|\log \left(\frac{\left|\Gamma_{G}\right|\left|\gamma_{B}\right|}{\left|\Gamma_{B}\right|\left|\gamma_{G}\right|}\right)\right| & =\left|\log \left(\frac{\left|T_{V}^{n+2}\left(\gamma_{G}\right)\right|\left|\gamma_{B}\right|}{\left|T_{V}^{n+2}\left(\gamma_{B}\right)\right|\left|\gamma_{G}\right|}\right)\right| \\
& =\left|\log \left(\frac{\left|D T_{V}^{n+2}\left(v_{G}\right)\right|}{\left|D T_{V}^{n+2}\left(v_{B}\right)\right|}\right)\right| \\
& =\left|\sum_{i=0}^{n+1}\left(\left.\log \left|D T_{V}\right|_{D T_{V}^{i}\left(v_{G}\right)}\left(T_{V}^{i}\left(p_{G}\right)\right)|-\log | D T_{V}\right|_{D T_{V}^{i}\left(v_{B}\right)}\left(T_{V}^{i}\left(p_{B}\right)\right) \mid\right)\right| \\
& \leq\left.\left.\sum_{i=0}^{n+1}|\log | D T_{V}\right|_{D T_{V}^{i}\left(v_{G}\right)}\left(T_{V}^{i}\left(p_{G}\right)\right)|-\log | D T_{V}\right|_{D T_{V}^{i}\left(v_{B}\right)}\left(T_{V}^{i}\left(p_{B}\right)\right)|| \\
& \leq\left.\left.\sum_{i=0}^{n+1}|| D T_{V}\right|_{D T_{V}^{i}\left(v_{G}\right)}\left(T_{V}^{i}\left(p_{G}\right)\right)|-| D T_{V}\right|_{D T_{V}^{i}\left(v_{B}\right)}\left(T_{V}^{i}\left(p_{B}\right)\right)|| .
\end{aligned}
$$

We estimate each of the terms in this sum using

Lemma 3.26. Suppose $f: \mathbb{R}^{n} \rightarrow \mathbb{R}^{n}$ is a smooth map, $a, b \in \mathbb{R}^{n}$, and $v_{a} \in T_{a} \mathbb{R}^{n}, v_{b} \in$ $T_{b} \mathbb{R}^{n}$ are unit vectors. Then

$$
\left.\left.|| D f\right|_{v_{a}}(a)|-| D f\right|_{v_{b}}(b)|| \leq\|f\|_{C^{2}}\left(\angle\left(v_{a}, v_{b}\right)+|a-b|\right) .
$$

Proof of Lemma 3.26.

$$
\begin{aligned}
\left.\left.|| D f\right|_{v_{a}}(a)|-| D f\right|_{v_{b}}(b)|| & \leq\left.\left.|| D f\right|_{v_{a}}(a)|-| D f\right|_{v_{b}}(a)||+\left.\left.|| D f\right|_{v_{b}}(a)|-| D f\right|_{v_{b}}(b)|| \\
& \leq\|D f(a)\| \cdot\left|v_{a}-v_{b}\right|+\|f\|_{C^{2}} \cdot|a-b| \\
& \leq\|f\|_{C^{2}}\left(\left|v_{a}-v_{b}\right|+|a-b|\right) \\
& \leq\|f\|_{C^{2}}\left(\angle\left(v_{a}, v_{b}\right)+|a-b|\right) .
\end{aligned}
$$

Now, Proposition 3.11 follows from Lemma 3.19.

\section{The Integrated Density of States}

4.1. Definition and basic properties. Recall the definition of $N(E, V)$ given in (1),

$$
N(E, V)=\lim _{n \rightarrow \infty} \frac{1}{n} N(E, \omega, V,[1, n]) .
$$


Proposition 4.1 (Hof, see [Ho]). For every $(E, V) \in \mathbb{R}^{2}$, the limit in (1) exists for every $\omega \in \mathbb{T}$ and its value does not depend on it.

The following proposition collects some well-known properties of the integrated density of states.

Proposition 4.2. (a) The map

$$
\mathbb{R} \times \mathbb{R} \ni(E, V) \mapsto N(E, V) \in[0,1]
$$

is continuous.

(b) For every $V \in \mathbb{R}$, there is a Borel measure on $\mathbb{R}$, called the density of states measure and denoted by $d N_{V}$, such that

$$
N(E, V)=\int_{\mathbb{R}} \chi_{(-\infty, E]} d N_{V} .
$$

(c) The topological support of the measure $d N_{V}$ is equal to $\Sigma_{V}$.

(d) The density of states measure is the $\omega$-average of the spectral measure associated with $H_{V, \omega}$ and the vector $\delta_{0} \in \ell^{2}(\mathbb{Z})$. That is, for every $V \in \mathbb{R}$ and every bounded measurable $g: \mathbb{R} \rightarrow \mathbb{R}$,

$$
\int_{\mathbb{R}} g d N_{V}=\int_{\omega \in \mathbb{T}}\left\langle\delta_{0}, g\left(H_{V, \omega}\right) \delta_{0}\right\rangle d \omega .
$$

(e) We have

$$
N(E, 0)= \begin{cases}0 & E \leq-2 \\ \frac{1}{\pi} \arccos \left(-\frac{E}{2}\right) & -2<E<2 \\ 1 & E \geq 2 .\end{cases}
$$

Proof. (a) This follows from (the proof of) Lemma 3.1 and Theorem 3.2 in [AS].

(b) For every $V \in \mathbb{R}$, the map

$$
\mathbb{R} \ni E \mapsto N(E, V) \in[0,1]
$$

is continuous by (a) and non-decreasing by construction, and hence it is the distribution function of a Borel measure on $\mathbb{R}$.

(c) and (d) See [CFKS, Sect. 9.2].

(e) This is folklore; see, for example, [LS, Theorem 1.1] and its discussion there for a simple derivation.

4.2. Complete gap labeling. Here we prove the following result, which implies Theorem 1.5 since the transversality assumption holds for $V_{0}>0$ sufficiently small.

Theorem 4.3. Suppose $V_{0}>0$ is such that for every $V \in\left(0, V_{0}\right]$ and every point in $\Omega_{V}$, its stable manifold intersects $\ell_{V}$ transversally. Then, for every $V \in\left(0, V_{0}\right]$, all gaps allowed by the gap labeling theorem are open. That is,

$$
\left\{N(E, V): E \in \mathbb{R} \backslash \Sigma_{V}\right\}=\{\{k \alpha\}: k \in \mathbb{Z}\} \cup\{1\} .
$$


Proof. Consider the preimages of the singularities of the trace map $F^{-1}\left(P_{i}\right), i=$ $1,2,3,4$, on the torus. They form a set of 4 periodic points of the hyperbolic automorphism $\mathcal{A}: \mathbb{T}^{2} \rightarrow \mathbb{T}^{2}$, and the stable manifolds of those periodic points intersect the line $\{\phi=0\}$ transversally at the points $\{k \alpha(\bmod 1)\},\left\{k \alpha+\frac{1}{2}(\bmod 1)\right\},\left\{k \alpha+\frac{\alpha}{2}(\bmod 1)\right\}$, and $\left\{k \alpha+\frac{1}{2}+\frac{\alpha}{2}(\bmod 1)\right\}$.

The images of these points under the semiconjugacy $F$ form the set of points on $\ell_{0}$ of the form $( \pm \cos (\pi m \alpha), \pm \cos (\pi m \alpha), 1)$, and they correspond to the energies $E \in$ $\{ \pm 2 \cos (\pi m \alpha), m \in \mathbb{Z}\}$. The integrated density of states for the free Laplacian, $N(E, 0)$, takes the values $\{\{m \alpha\}: m \in \mathbb{Z}\}$ at these energies.

After we increase the value of the coupling constant, each singularity splits into two periodic points, and each of the stable manifolds of the singularities splits into two strong stable manifolds of the periodic points. Every point between the stable manifolds has an unbounded positive semiorbit, and therefore the interval that those manifolds cut in the line $\ell_{V}$ corresponds to a gap in the spectrum.

Due to the continuous dependence of $N(E, V)$ on the coupling constant and the local constancy of $N(\cdot, V)$ in the complement of $\Sigma_{V}$, the integrated density of states takes the same value in the formed gap as at the energy that corresponds to the initial point of intersection of the stable manifold of singularity with $\ell_{0}$.

\subsection{More on the asymptotic gap lengths.}

Proof of Theorem 1.6. Fix any $m \in \mathbb{Z} \backslash\{0\}$. The integrated density of states of the free Laplacian takes the values $\{ \pm m \alpha\}$ at the energies $\{ \pm 2 \cos \pi m \alpha\}$.

If $m=2 k$, then these energies correspond to points with $\theta$-coordinates $k \alpha(\bmod 1)$ and $k \alpha+\frac{1}{2}(\bmod 1)$ on $F^{-1}\left(\ell_{0}\right)$.

If $m=2 k+1$, then these energies correspond to points with $\theta$-coordinates $k \alpha+$ $\frac{\alpha}{2}(\bmod 1)$ and $k \alpha+\frac{\alpha}{2}+\frac{1}{2}(\bmod 1)$ on $F^{-1}\left(\ell_{0}\right)$.

Take one of these points, $Q_{k} \in F^{-1}\left(\ell_{0}\right)$. Let $P^{*}$ be the singularity such that $Q_{k} \in$ $F^{-1}\left(W^{s s}\left(P^{*}\right)\right)$. Denote $\Gamma=F^{-1}\left(W_{1}^{s s}\left(P^{*}\right)\right)$. Let $M \in \mathbb{N}$ be the smallest number such that $\mathcal{A}^{-M}(\Gamma)$ contains $Q_{k}$.

Then $\mathcal{A}^{M}\left(F^{-1}\left(\ell_{0}\right)\right)$ intersects $\Gamma$ at some point $Z$, and the distance from $Z$ to the set of singularities is uniformly (in $|m|$ ) bounded from zero.

Denote by $P^{\prime}(V)$ and $P^{\prime \prime}(V)$ the periodic points on $S_{V}$ near $P^{*}$ (of period 2 or of period 6 , depending on $\left.P^{*}\right)$. Denote by $\Gamma^{\prime}=F_{V}^{-1}\left(W_{1}^{s s}\left(P^{\prime}(V)\right)\right.$ and $\Gamma^{\prime \prime}=$ $F_{V}^{-1}\left(W_{1}^{s s}\left(P^{\prime \prime}(V)\right)\right.$.

Then $\widetilde{T}_{V}^{M}\left(F_{V}^{-1}\left(\ell_{V}\right)\right)$ (recall that $\widetilde{T}_{V}$ was defined by $\left.(24)\right)$ intersects $\Gamma^{\prime}$ and $\Gamma^{\prime \prime}$ transversally near the point $Z$ and the curves $\Gamma^{\prime}$ and $\Gamma^{\prime \prime}$ cut in $\widetilde{T}_{V}^{M}\left(F_{V}^{-1}\left(l_{V}\right)\right)$ an interval $I_{V}$ whose length is of order $V$. In other words, $\lim _{V \rightarrow 0} \frac{\left|I_{V}\right|}{|V|}$ exists and is uniformly bounded from zero and from above.

We also have

$$
\lim _{V \rightarrow 0} \frac{\widetilde{T}_{V}^{-M}\left(I_{V}\right)}{\left|I_{V}\right|}=\left.\left|D \mathcal{A}^{M}\right|_{F^{-1}\left(l_{0}\right)}\right|^{-1}=\alpha^{M} \cdot C\left(Q_{k}\right),
$$

where $C\left(Q_{k}\right)$ is bounded from zero and from above.

Notice that if $\mathcal{A}^{-M}(\Gamma)$ intersects $F^{-1}\left(\ell_{0}\right)$ at $Q_{k}$, then $\mathcal{A}^{-M}(\Gamma)$ must be of length at least $|k| \alpha^{-1}$. On the other hand, $\left|\mathcal{A}^{-M}(\Gamma)\right|=\alpha^{-M}|\Gamma|$. Therefore, $\alpha^{-M}|\Gamma| \geq|k| \alpha^{-1}$. 
Hence

$$
\lim _{V \rightarrow 0} \frac{\left|\widetilde{T}_{V}^{-M}\left(I_{V}\right)\right|}{|V|}=\lim _{V \rightarrow 0} \frac{\left|\widetilde{T}_{V}^{-M}\left(I_{V}\right)\right|}{\left|I_{V}\right|} \frac{\left|I_{V}\right|}{|V|}=\alpha^{M} C\left(Q_{k}\right) \lim _{V \rightarrow 0} \frac{\left|I_{V}\right|}{|V|} \leq \bar{C}\left(Q_{k}\right) \frac{1}{|k|} .
$$

On the other hand,

$$
\lim _{V \rightarrow 0} \frac{\left|U_{m}(V)\right|}{\left|\widetilde{T}_{V}^{-M}\left(I_{V}\right)\right|}=|D F|_{F^{-1}\left(l_{0}\right)}\left(Q_{k}\right) \mid
$$

is bounded from above (since $\|D F\|$ is bounded). Therefore we have

$$
\lim _{V \rightarrow 0} \frac{\left|U_{m} V\right|}{|V|}=\lim _{V \rightarrow 0} \frac{\left|\widetilde{T}_{V}^{-M}\left(I_{V}\right)\right|}{|V|} \frac{\left|U_{m}(V)\right|}{\left|\widetilde{T}_{V}^{-M}\left(I_{V}\right)\right|} \leq \overline{\bar{C}}\left(Q_{k}\right) \frac{1}{|k|},
$$

where $\overline{\bar{C}}\left(Q_{k}\right)$ is uniformly bounded from above.

Remark 4.4. Notice that one can actually claim a bit more. Namely, for those gaps that appear away from the endpoints of the free spectrum, the corresponding constant $C_{m}$ is uniformly bounded away from zero. This follows from the fact that away from the singularities, the differential of $F$ has norm which is bounded away from zero.

\section{Spectral Measures and Transport Exponents}

5.1. Solution estimates. In this subsection we study solutions to the difference equation and prove upper and lower bounds for them. Results of this kind are known (we provide the references below), but our purpose here is to obtain explicit quantitative estimates, as functions of the coupling constant, as they enter explicitly in the bounds on fractal dimensions of spectral measures and transport exponents and we wish to prove the best bounds possible for the latter quantities to get an idea about their behavior at weak coupling. These applications will be discussed in the next two subsections.

Denote the largest root of the polynomial $x^{3}-(2+V) x-1$ by $a_{V}$. For small $V>0$, we have $a_{V} \approx \frac{\sqrt{5}+1}{2}+c V$ with a suitable constant $c$. Our goal is to prove the following pair of theorems (recall that $\sigma\left(H_{V, \omega}\right)=\Sigma_{V}$ for every $\omega$ ).

Let $M(n, m, \omega, E)$ be the standard transfer matrix, that is, the $\operatorname{SL}(2, \mathbb{R})$ matrix that maps $(u(m+1), u(m))^{T}$ to $(u(n+1), u(n))^{T}$ for every solution $u$ of the difference equation $H_{V, \omega} u=E u$.

Theorem 5.1. For every $V>0$ and every

$$
\zeta>\frac{\log \left[(5+2 V)^{1 / 2}(3+V) a_{V}\right]}{\log \frac{\sqrt{5}+1}{2}}
$$

there is a constant $C>0$ such that for every $\omega$ and every $E \in \Sigma_{V}$, we have

$$
\max _{0 \leq|n-m| \leq N}\|M(n, m, \omega, E)\| \leq C N^{\zeta}
$$


Theorem 5.2. For every $V>0$,

$$
\gamma_{\ell}<\frac{\log \left(1+\frac{1}{(2+2 V)^{2}}\right)}{16 \cdot \log \left(\frac{\sqrt{5}+1}{2}\right)}, \quad \text { and } \quad \gamma_{u}>1+\frac{\log \left[(5+2 V)^{1 / 2}(3+V) a_{V}\right]}{\log \frac{\sqrt{5}+1}{2}} \text {, }
$$

there are constants $C_{\ell}, C_{u}>0$ such that for every $\omega, L \geq 1$, and $E \in \Sigma_{V}$, we have that every solution to the difference equation $H_{V, \omega} u=E u$, which is normalized by $|u(0)|^{2}+|u(1)|^{2}=1$, obeys the estimates

$$
C_{\ell} L^{\gamma_{\ell}} \leq\|u\|_{L} \leq C_{u} L^{\gamma_{u}},
$$

where the local $\ell^{2}$ norm $\|\cdot\|_{L}$ is defined by

$$
\|u\|_{L}^{2}=\sum_{n=1}^{[L]}|u(n)|^{2}+(L-[L])|u([L]+1)|^{2}
$$

for $L \geq 1$.

Theorem 5.1 is a quantitative version of [DL99a, Theorem 3], which in turn was a generalization of [IT, Theorem 1]. Theorem 5.2, on the other hand, is a quantitative version of [DKL, Props. 5.1 and 5.2]. The upper bound in Theorem 5.2 is a consequence of Theorem 5.1, while the lower bound in Theorem 5.2 will be extracted from the details of the proof of [DKL, Prop. 5.1].

Let us recall some notation from [DL99a]. For $V>0, E \in \mathbb{R}$, and $a \in\{0,1\}$, we write

$$
T(V, E, a)=\left(\begin{array}{cc}
E-V a & -1 \\
1 & 0
\end{array}\right) .
$$

For a word $w=w_{1} \ldots w_{n} \in\{0,1\}^{*}$, we then set

$$
M(V, E, w)=T\left(V, E, w_{n}\right) \times \cdots \times T\left(V, E, w_{1}\right) .
$$

Next, define the words $\left\{s_{n}\right\}_{n \geq 0}$ inductively by

$$
s_{0}=0, \quad s_{1}=1, \quad s_{n}=s_{n-1} s_{n-2} \quad \text { for } n \geq 2 .
$$

By the definition of these words, there is a unique $u \in\{0,1\}^{\mathbb{Z}_{+}}$(the fixed point of the Fibonacci substitution) that has $s_{n}$ as a prefix for every $n \geq 1$; namely $u=1011010110110 \ldots$ We denote the set of finite subwords of $u$ by $\mathcal{W}_{u}$, that is, $\mathcal{W}_{u}=\{0,1,01,10,11,010,011,101,110, \ldots\}$. By uniform recurrence of $u$ it suffices to consider the matrices $M(V, E, w)$ for $E \in \Sigma_{V}$ and $w \in \mathcal{W}_{u}$ when proving Theorems 5.1 and 5.2.

With these quantities, we have

$$
M_{n}=M_{n}(V, E)=M\left(V, E, s_{n}\right)
$$

and consequently

$$
x_{n}=x_{n}(V, E)=\frac{1}{2} \operatorname{Tr} M\left(V, E, s_{n}\right)
$$

for $n \geq 1$. 
Finding upper bounds for the norm of transfer matrices consists of three steps. The first step is to bound the special matrices $M_{n}$, that is, $M\left(F_{n}, 0, \omega=0, E\right)$. This is the objective of Lemma 5.3. Then, in Lemma 5.5, we use interpolation to bound the norm of the matrices $M(n, 0, \omega=0, E)$. Finally, the case of general matrices $M(n, m, \omega, E)$ is treated using partition. This will then complete the proof of Theorem 5.1.

We begin with the first step. Part (a) of the following lemma is due to Süto (see [S87, Lemma 2]) and part (b) is a relative of [IT, Lemma 4.(ii)]:

Lemma 5.3. (a) We have $E \in \Sigma_{V}$ if and only if the sequence $\left\{x_{n}\right\}$ is bounded. Moreover, we have

$$
\left|x_{n}\right| \leq 1+\frac{V}{2}
$$

for every $E \in \Sigma_{V}$ and $n \geq 1$.

(b) With some positive $V$-dependent constant $C$, we have

$$
\left\|M_{n}\right\| \leq C a_{V}^{n}
$$

for every $E \in \Sigma_{V}$ and $n \geq 1$.

Proof. As pointed out above, part (a) is known. Let us prove part (b). By the CayleyHamilton Theorem, we have that $M_{n}^{2}-2 x_{n} M_{n}+I=0$, that is,

$$
M_{n}=2 x_{n} I-M_{n}^{-1} \text {. }
$$

The recursion for the matrices $M_{n}, M_{n}=M_{n-2} M_{n-1}$, gives

$$
M_{n-2}^{-1}=M_{n-1} M_{n}^{-1} \text {. }
$$

Putting these things together, we find

$$
M_{n}=M_{n-2} M_{n-1}=M_{n-2}\left(2 x_{n-1} I-M_{n-1}^{-1}\right)=2 x_{n-1} M_{n-2}-M_{n-3}^{-1} .
$$

Since we also have $\left\|M_{n}\right\|=\left\|M_{n}^{-1}\right\|$, we obtain from this identity along with part (a) the estimate

$$
\left\|M_{n}\right\| \leq(2+V)\left\|M_{n-2}\right\|+\left\|M_{n-3}\right\|
$$

for every $E \in \Sigma_{V}$.

Consider for comparison the recursion

$$
m_{n}=(2+V) m_{n-2}+m_{n-3} .
$$

It is clear that we have $\left\|M_{n}\right\| \leq m_{n}$ if we consider the sequence $\left\{m_{n}\right\}$ generated by the recursion and the initial conditions $m_{1}=\left\|M_{1}\right\|, m_{2}=\left\|M_{2}\right\|, m_{3}=\left\|M_{3}\right\|$.

Any solution of the recursion is of the form

$$
m_{n}=c_{1} x_{1}^{n}+c_{2} x_{2}^{n}+c_{3} x_{3}^{n},
$$

where the $x_{j}$ are the roots of the characteristic polynomial $x^{3}-(2+V) x-1$. Thus, the definition of $a_{V}$ implies that the estimate in (b) holds. 
Remark. Since $F_{n} \sim\left(\frac{\sqrt{5}+1}{2}\right)^{n}$, we can infer that $\left\|M_{n}\right\| \leq C F_{n}^{1+\delta}$ with $\delta \rightarrow 0$ as $V \rightarrow 0$. Since for $V=0$, the norm of the transfer matrices grows linearly at the energies $E= \pm 2$, we cannot expect a better result in general. However, since the transfer matrices are bounded when $V=0$ and $E \in(-2,2)$, it is reasonable to expect that there is a better bound for small values of $V$ for most energies in the spectrum.

Let us now turn to the second step, which is the interpolation of the estimates from the previous lemma to non-Fibonacci sites in the $\omega=0$ sequence. The following lemma is a relative of [IT, Lemma 5]. Even though the proof is closely related to that in [IT], we give the details to clearly show where the improved estimate comes from.

Lemma 5.4. For $n \geq 1$ and $k \geq 0$, we may write

$$
M_{n} M_{n+k}=P_{k}^{(1)} M_{n+k}+P_{k}^{(2)} M_{n+k-1}+P_{k}^{(3)} M_{n+k-2}+P_{k}^{(4)} I
$$

with coefficients $P_{k}^{(j)}$ that also depend on $n$ and $E$. Moreover, for every $n \geq 1$ and $E \in \Sigma_{V}$, we have that

$$
\sum_{j=1}^{4}\left|P_{k}^{(j)}\right| \leq(5+2 V)(3+V)^{\lfloor k / 2\rfloor} .
$$

Proof. Consider the case $k=0$. Then,

$$
M_{n} M_{n}=M_{n}^{2}=2 x_{n} M_{n}-I
$$

by Cayley-Hamilton and hence we can set

$$
P_{0}^{(1)}=2 x_{n}, \quad P_{0}^{(2)}=P_{0}^{(3)}=0, \quad P_{0}^{(4)}=-1 .
$$

It follows that for $E \in \Sigma_{V}$, we have the estimate

$$
\sum_{j=1}^{4}\left|P_{0}^{(j)}\right| \leq 3+V
$$

Consider now the case $k=1$. Then,

$$
M_{n} M_{n+1}=M_{n+2}=2 x_{n+1} M_{n}+M_{n-1}-2 x_{n-1} I
$$

by [IT, Lemma 6] and hence we can set

$$
P_{1}^{(1)}=0, \quad P_{1}^{(2)}=2 x_{n+1}, \quad P_{1}^{(3)}=1, \quad P_{1}^{(4)}=-2 x_{n-1} .
$$

For $E \in \Sigma_{V}$, we therefore have

$$
\sum_{j=1}^{4}\left|P_{1}^{(j)}\right| \leq 5+2 V
$$


Next, assume that the lemma holds for $k \in\{1, \ldots, \ell\}$ and consider the case $k=\ell+1$. Then, using [IT, Lemma 6] one more time, we find

$$
\begin{aligned}
M_{n} M_{n+\ell+1}= & \left(M_{n} M_{n+\ell-1}\right) M_{n+\ell} \\
= & \left(P_{\ell-1}^{(1)} M_{n+\ell-1}+P_{\ell-1}^{(2)} M_{n+\ell-2}+P_{\ell-1}^{(3)} M_{n+\ell-3}+P_{\ell-1}^{(4)} I\right) M_{n+\ell} \\
= & P_{\ell-1}^{(1)} M_{n+\ell+1}+P_{\ell-1}^{(2)}\left(2 x_{n+\ell-2} M_{n+\ell}-M_{n+\ell-1}\right) \\
& +P_{\ell-1}^{(3)}\left(2 x_{n+\ell-1} M_{n+\ell-1}-I\right)+P_{\ell-1}^{(4)} M_{n+\ell} \\
= & P_{\ell+1}^{(1)} M_{n+\ell+1}+P_{\ell+1}^{(2)} M_{n+\ell}+P_{\ell+1}^{(3)} M_{n+\ell-1}+P_{k}^{(4)} I,
\end{aligned}
$$

where we set

$$
\begin{aligned}
& P_{\ell+1}^{(1)}=P_{\ell-1}^{(1)} \\
& P_{\ell+1}^{(2)}=2 x_{n+\ell-2} P_{\ell-1}^{(2)}+P_{\ell-1}^{(4)} \\
& P_{\ell+1}^{(3)}=-P_{\ell-1}^{(2)}+2 x_{n+\ell-1} P_{\ell-1}^{(3)} \\
& P_{\ell+1}^{(4)}=-P_{\ell-1}^{(3)} .
\end{aligned}
$$

It follows that

$$
\sum_{j=1}^{4}\left|P_{\ell+1}^{(j)}\right| \leq(3+V) \sum_{j=1}^{4}\left|P_{\ell-1}^{(j)}\right| .
$$

By induction hypothesis, we obtain the desired estimate.

Lemma 5.5. For every $V>0$ and every $\zeta$ obeying (29), there is a constant $\tilde{C}$ so that

$$
\|M(n, 0, \omega=0, E)\| \leq \tilde{C} n^{\zeta}
$$

for every $n \geq 1, E \in \Sigma_{V}$.

Before we give the proof of Lemma 5.5, let us recall some facts about the minimal representation of positive integers in terms of Fibonacci numbers; compare, for example, [HCB,Le,Z]. Given $n \geq 1$, there is a unique representation

$$
n=\sum_{k=0}^{K} F_{n_{k}}
$$

such that $n_{k} \in \mathbb{Z}_{+}$and $n_{k+1}-n_{k} \geq 2$. This is the so-called Zeckendorf representation of $n$ (which, incidentally, was published by Lekkerkerker some twenty years before Zeckendorf). It is found by the greedy algorithm, that is, $F_{n_{K}}$ is set to be the largest Fibonacci number less than or equal to $n, n$ is replaced by $n-F_{n_{K}}$, and the process is repeated until we have a zero remainder. The property $n_{k+1}-n_{k} \geq 2$ follows from the way the algorithm works together with the recursion for the Fibonacci numbers. Uniqueness of this representation in turn follows from $n_{k+1}-n_{k} \geq 2$.

By construction, we have

$$
F_{n_{K}} \leq n<F_{n_{K}+1} \leq 2 F_{n_{K}} .
$$


We will also need a relation between $n$ and $K$, that is, the number of terms in the Zeckendorf representation of $n$. The algorithm to compute $K=K(n)$ is the following: Start with two words over the positive integers, 1 and 1 . The next word is obtained by writing down the previous word, following by the word preceding the previous word, but this time with every symbol increased by one. Now iterate this procedure:

$$
1, \quad 1, \quad 12, \quad 122, \quad 12223, \quad 12223233, \quad 1222323323334, \ldots
$$

Concatenate all the words to obtain a semi-infinite word over the positive integers:

$$
111212212223122232331222323323334 \ldots
$$

The $n^{\text {th }}$ symbol in this semi-infinite sequence is $K(n)$. Notice that the lengths of these words follow the Fibonacci sequence. Moreover, only every other word has an increase in the maximum value of its entries, relative to the previous one, and the increase is by one whenever it happens. This shows that

$$
F_{2(K-1)} \leq n
$$

Proof of Lemma 5.5. From the Zeckendorf representation of $n \geq 1$, one finds that

$$
M(n, 0, \omega=0, E)=M_{n_{0}} M_{n_{1}} \cdots M_{n_{K}} ;
$$

compare the beginning of the proof of [IT, Theorem 1].

Let us prove by induction that with

$$
b_{V}=(3+V)^{1 / 2}
$$

we have for every $k \geq 1$,

$$
\left\|M_{n_{0}} M_{n_{1}} \cdots M_{n_{k}}\right\| \leq C(5+2 V)^{k} b_{V}^{-n_{0}+2(k-1)}\left(a_{V} b_{V}\right)^{n_{k}}
$$

Consider first the case $k=1$. From Lemmas 5.3 and 5.4, we may infer that

$$
\begin{aligned}
\left\|M_{n_{0}} M_{n_{1}}\right\| & \leq\left|P_{n_{1}-n_{0}}^{(1)}\right| \cdot\left\|M_{n_{1}}\right\|+\left|P_{n_{1}-n_{0}}^{(2)}\right| \cdot\left\|M_{n_{1}-1}\right\|+\left|P_{n_{1}-n_{0}}^{(3)}\right| \cdot\left\|M_{n_{1}-2}\right\|+\left|P_{n_{1}-n_{0}}^{(4)}\right| \\
& \leq \sum_{j=1}^{4}\left|P_{n_{1}-n_{0}}^{(j)}\right| C a_{V}^{n_{1}} \\
& \leq C(5+2 V)(3+V)^{\left\lfloor\left(n_{1}-n_{0}\right) / 2\right\rfloor} a_{V}^{n_{1}} \\
& \leq C(5+2 V) b_{V}^{-n_{0}}\left(a_{V} b_{V}\right)^{n_{1}} .
\end{aligned}
$$

Thus, the estimate (34) holds for $k=1$. 
Now assume that (34) holds for $k \in\{1, \ldots, \ell\}$ and consider the case $k=\ell+1$. Using Lemma 5.4 again, we see that

$$
\begin{aligned}
\left\|M_{n_{0}} M_{n_{1}} \cdots M_{n_{\ell}} M_{n_{\ell+1}}\right\|= & \left\|\left(M_{n_{0}} M_{n_{1}}\right) M_{n_{2}} \cdots M_{n_{\ell}} M_{n_{\ell+1}}\right\| \\
\leq & \left|P_{n_{1}-n_{0}}^{(1)}\right| \cdot\left\|M_{n_{1}} M_{n_{2}} \cdots M_{n_{\ell}} M_{n_{\ell+1}}\right\| \\
& +\left|P_{n_{1}-n_{0}}^{(2)}\right| \cdot\left\|M_{n_{1}-1} M_{n_{2}} \cdots M_{n_{\ell}} M_{n_{\ell+1}}\right\| \\
& +\left|P_{n_{1}-n_{0}}^{(3)}\right| \cdot\left\|M_{n_{1}-2} M_{n_{2}} \cdots M_{n_{\ell}} M_{n_{\ell+1}}\right\| \\
& +\left|P_{n_{1}-n_{0}}^{(4)}\right| \cdot\left\|M_{n_{2}} \cdots M_{n_{\ell}} M_{n_{\ell+1}}\right\| \\
\leq & \left|P_{n_{1}-n_{0}}^{(1)}\right| \cdot C(5+2 V)^{\ell} b_{V}^{-n_{1}+2(\ell-1)}(a b)^{n_{\ell+1}} \\
& +\left|P_{n_{1}-n_{0}}^{(2)}\right| \cdot C(5+2 V)^{\ell} b_{V}^{-n_{1}+1+2(\ell-1)}\left(a_{V} b_{V}\right)^{n_{\ell+1}} \\
& +\left|P_{n_{1}-n_{0}}^{(3)}\right| \cdot C(5+2 V)^{\ell} b_{V}^{-n_{1}+2+2(\ell-1)}\left(a_{V} b_{V}\right)^{n_{\ell+1}} \\
& +\left|P_{n_{1}-n_{0}}^{(4)}\right| \cdot C(5+2 V)^{\ell} b_{V}^{-n_{2}+2(\ell-2)}\left(a_{V} b_{V}\right)^{n_{\ell+1}} \\
\leq & \left(\sum_{j}^{4}\left|P_{n_{1}-n_{0}}^{(j)}\right|\right) C(5+2 V)^{\ell} b_{V}^{-n_{1}+2 \ell}\left(a_{V} b_{V}\right)^{n_{\ell+1}} \\
\leq & (5+2 V) b^{n_{1}-n_{0}} C(5+2 V)^{\ell} b_{V}^{-n_{1}+2 \ell}\left(a_{V} b_{V}\right)^{n_{\ell+1}} \\
= & C(5+2 V)^{\ell+1} b_{V}^{-n_{0}+2((\ell+1)-1)}\left(a_{V} b_{V}\right)^{n_{\ell+1}} .
\end{aligned}
$$

We conclude that (34) holds for $k=\ell+1$.

Therefore,

$$
\|M(n, 0, \omega=0, E)\| \leq C(5+2 V)^{K} b_{V}^{-n_{0}+2(K-1)}\left(a_{V} b_{V}\right)^{n_{K}} .
$$

It follows that

$$
\begin{aligned}
& \limsup _{n \rightarrow \infty} \frac{\log \|M(n, 0, \omega=0, E)\|}{\log n} \\
& \leq \limsup _{n \rightarrow \infty} \frac{\log \left(C(5+2 V)^{K} b_{V}^{-n_{0}+2(K-1)}\left(a_{V} b_{V}\right)^{n_{K}}\right)}{\log n} \\
& \leq \limsup _{n \rightarrow \infty} \frac{K \log (5+2 V)+2 K \log b_{V}+n_{K} \log \left(a_{V} b_{V}\right)}{\log n} \\
& \leq \limsup _{n \rightarrow \infty} \frac{\frac{\log n}{2 \log \frac{\sqrt{5}+1}{2}} \log (5+2 V)+2 \frac{\log n}{2 \log \frac{\sqrt{5}+1}{2}} \log b_{V}+\frac{\log n}{\log \frac{\sqrt{5}+1}{2}} \log \left(a_{V} b_{V}\right)}{\log n} \\
& =\frac{\log (5+2 V)^{1 / 2}+\log b_{V}+\log \left(a_{V} b_{V}\right)}{\log \frac{\sqrt{5}+1}{2}} \\
& =\frac{\log \left[(5+2 V)^{1 / 2}(3+V) a_{V}\right]}{\log \frac{\sqrt{5}+1}{2}}
\end{aligned}
$$

where we used (32) and (33) in the third step. Since these estimates are uniform in $E \in \Sigma_{V}$, the result follows. 
In the third step, we can now turn to the

Proof of Theorem 5.1. Suppose $V>0$ and $\zeta$ obeys (29). We will prove the following estimate:

$$
\|M(V, E, w)\| \leq C|w|^{\zeta} \quad \text { for every } V>0, E \in \Sigma_{V} \text {, and } w \in \mathcal{W}_{u},
$$

from which (30) immediately follows.

Let $V, E, w$ as in (35) be given. As explained in [DL99a, Proof of Lemma 5.2], we can write

$$
w=x y z
$$

where $y \in\{a, b\}^{*}$ is a word of length 2 and $x^{R}$ (the reversal of $x$ ) and $z$ are prefixes of $u$. Thus,

$$
\|M(V, E, w)\| \leq\|M(V, E, x)\| \cdot\|M(V, E, y)\| \cdot\|M(V, E, z)\| .
$$

It follows from [DL99a, Lemma 5.1] that

$$
\left\|M\left(V, E, x^{R}\right)\right\|=\|M(V, E, x)\| .
$$

Moreover, Lemma 5.5 yields

$$
\left\|M\left(V, E, x^{R}\right)\right\| \leq \tilde{C}|x|^{\zeta}
$$

and

$$
\|M(V, E, z)\| \leq \tilde{C}|z|^{\zeta}
$$

Since $\zeta>1$ and $y$ has length 2, (35) follows from (36)-(39).

Proof of Theorem 5.2. The upper bound in (31) follows from Theorem 5.1. The lower bound in (31) will be extracted here from [DKL] since it is not made explicit there.

Denote $U(n)=(u(n+1), u(n))^{T}$ and consider the associated local $\ell^{2}$-norms $\|U\|_{L}$. Power-law bounds for $\|U\|_{L}$ correspond in a natural way to power-law bounds for $\|u\|_{L}$, so let us discuss the former object. By considering squares adjacent to the origin and Gordon's two-block method, Damanik, Killip, and Lenz showed that

$$
\|U\|_{F_{8 n}} \geq\left(1+\frac{1}{(2+2 V)^{2}}\right)^{n / 2}
$$

see [DKL, Lemma 4.1]. We find

$$
\liminf _{n \rightarrow \infty} \frac{\log \|U\|_{F_{8(n-1)}}}{\log F_{8 n}} \geq \frac{\log \left(1+\frac{1}{(2+2 V)^{2}}\right)}{16 \cdot \log \left(\frac{\sqrt{5}+1}{2}\right)}
$$

uniformly in $\omega \in \mathbb{T}$ and $E \in \Sigma_{V}$. This, together with monotonicity, yields the asserted lower bound. 
5.2. Spectral measures. Given $V>0$ and $\omega \in \mathbb{T}$, let us consider the operator $H_{V, \omega}$. Since this operator is self-adjoint, the spectral theorem associates with each $\psi \in \ell^{2}(\mathbb{Z})$ a Borel measure $\mu_{\psi}$ that is supported by $\Sigma_{V}$ and obeys

$$
\left\langle\psi, g\left(H_{V, \omega}\right) \psi\right\rangle=\int g(E) d \mu_{\psi}(E)
$$

for every bounded measurable function $g$ on $\Sigma_{V}$. It follows from Theorem 5.2 that each of these spectral measures gives zero weight to sets of small Hasudorff dimension. More precisely, we have the following result:

Corollary 1. For every $V>0$, every $\omega$, and every

$$
\alpha<\frac{2 \log \left(1+\frac{1}{(2+2 V)^{2}}\right)}{\log \left(1+\frac{1}{(2+2 V)^{2}}\right)+16 \cdot \log \left(\frac{\sqrt{5}+1}{2}\right)+16 \log \left[(5+2 V)^{1 / 2}(3+V) a_{V}\right]},
$$

we have that $\mu_{\psi}(S)=0$ for every $\psi \in \ell^{2}(\mathbb{Z})$ and every Borel set $S$ with $h^{\alpha}(S)=0$.

Proof. This is an immediate consequence of Theorem 5.2 and [DKL, Theorem 1].

5.3. Transport exponents. Absolute continuity of spectral measures with respect to Hausdorff measures is important because it implies lower bounds for transport exponents via the Guarneri-Combes-Last Theorem. Let us recall this connection briefly. Given $H_{V, \omega}$ and $\psi$ as above, consider the Schrödinger equation $i \partial_{t} \psi(t)=H_{V, \omega} \psi(t)$ with initial condition $\psi(0)=\psi$. Then, the unique solution of this initial-value problem is given by $\psi(t)=e^{-i t H_{V, \omega}} \psi$. To measure the spreading of a wavepacket, one considers time-averaged moments of the position operator. This is of course mainly of interest when $\psi(0)=\psi$ is well-localized and in fact, in $\ell^{2}(\mathbb{Z})$ one usually considers the canonical initial state $\psi=\delta_{0}$.

One is interested in the growth of $\left\langle\left\langle|X|_{\psi}^{p}\right\rangle\right\rangle(T)$ as $T \rightarrow \infty$ for $p>0$, where

$$
\left\langle|X|_{\psi}^{p}\right\rangle(t)=\sum_{n \in \mathbb{Z}}|n|^{p}\left|\left\langle\delta_{n}, e^{-i t H_{V, \omega}} \psi\right\rangle\right|^{2}
$$

and the time average of a $t$-dependent function $f$ is given by either

$$
\langle f\rangle(T)=\frac{1}{T} \int_{0}^{T} f(t) d t
$$

or

$$
\langle f\rangle(T)=\frac{2}{T} \int_{0}^{\infty} e^{-2 t / T} f(t) d t .
$$

We will indicate in the results below which time-average is involved. However, for compactly supported (or fast-decaying) $\psi$, all the results mentioned below hold for both types of time-average.

To measure the power-law growth of $\left\langle\left\langle|X|^{p}\right\rangle\right\rangle(T)$, define

$$
\beta_{\psi}^{+}(p)=\limsup _{T \rightarrow \infty} \frac{\log \left\langle\left\langle|X|_{\psi}^{p}\right\rangle\right\rangle(T)}{p \log T}
$$


and

$$
\beta_{\psi}^{-}(p)=\liminf _{T \rightarrow \infty} \frac{\log \left\langle\left\langle|X|_{\psi}^{p}\right\rangle\right\rangle(T)}{p \log T} .
$$

The Guarneri-Combes-Last Theorem (see [La, Theorem 6.1]) states that if $\mu_{\psi}$ is not supported by a Borel set $S$ with $h^{\alpha}(S)=0$, then $\beta^{ \pm}(p) \geq \alpha$ for every $p>0$, where the time-average is given by (40).

Corollary 2. For every $V>0, \omega \in \mathbb{T}, 0 \neq \psi \in \ell^{2}(\mathbb{Z})$, and $p>0$, we have

$$
\beta_{\psi}^{ \pm}(p) \geq \frac{2 \log \left(1+\frac{1}{(2+2 V)^{2}}\right)}{\log \left(1+\frac{1}{(2+2 V)^{2}}\right)+16 \cdot \log \left(\frac{\sqrt{5}+1}{2}\right)+16 \log \left[(5+2 V)^{1 / 2}(3+V) a_{V}\right]},
$$

where the time-average is given by (40).

Proof. Immediate from the Guarneri-Combes-Last Theorem and Corollary 1.

We also have the following estimate, which holds for a special initial state but which is better when $p$ is large:

Corollary 3. For every $V>0, \omega \in \mathbb{T}$, and $p>0$, we have

$$
\begin{aligned}
\beta_{\delta_{0}}^{ \pm}(p) \geq & \frac{\log \frac{\sqrt{5}+1}{2}}{\log \frac{\sqrt{5}+1}{2}+\log \left[(5+2 V)^{1 / 2}(3+V) a_{V}\right]} \\
& -\frac{3 \log \left[(5+2 V)^{1 / 2}(3+V) a_{V}\right]}{p\left(\log \frac{\sqrt{5}+1}{2}+\log \left[(5+2 V)^{1 / 2}(3+V) a_{V}\right]\right)},
\end{aligned}
$$

where the time-average is given by (41).

Proof. This is a consequence of Theorem 5.1 and results of Damanik, Sütô, and Tcheremchantsev (see [DST, Theorem 1] and compare also [DT08, Theorem 1]).

It can be shown that for compactly supported (or fast-decaying) $\psi$, the transport exponents $\beta_{\psi}^{ \pm}(p)$ are non-decreasing functions of $p$ taking values in $[0,1]$. Thus, in this case it is natural to consider the limits of these quantities as $p \downarrow 0$ and $p \uparrow \infty$ and denote them by $\beta_{\psi}^{ \pm}(0)$ and $\beta_{\psi}^{ \pm}(\infty)$, respectively. The bounds in the two previous corollaries imply estimates for $\beta_{\psi}^{ \pm}(0)$ and $\beta_{\psi}^{ \pm}(\infty)$ as well. However, at small coupling, a better estimate for $\beta_{\delta_{0}}^{ \pm}(\infty)$ is obtained via a different route:

Corollary 4. We have

$$
\lim _{V \downarrow 0} \beta_{\delta_{0}}^{ \pm}(\infty)=\lim _{V \downarrow 0} \lim _{p \uparrow \infty} \beta_{\delta_{0}}^{ \pm}(p)=1,
$$

uniformly in $\omega \in \mathbb{T}$. Here, the time-average is given by (41).

Proof. Damanik, Embree, Gorodetski, and Tcheremchantsev showed for the Fibonacci Hamiltonian that $\beta_{\delta_{0}}^{ \pm}(\infty) \geq \operatorname{dim}_{B}^{ \pm}\left(\Sigma_{V}\right)$ for every $V>0$ and $\omega \in \mathbb{T}$; see [DEGT, Theorem 3]. Since the right-hand side converges to one as $V \downarrow 0$, the result follows. 
Remarks. (a) As pointed out above, while the papers [DEGT,DST,DT03] work with the time-average (41), the results above carry over to transport exponents defined using (40).

(b) We find that $\beta_{\delta_{0}}^{ \pm}(\infty)$, considered as a function of $V$, extends continuously to zero. While we would expect this also for other initial states $\psi$, it does not follow from Corollary 2. It would be interesting to extend this continuity result to all fast-decaying initial states or to exhibit one for which it fails.

\section{Consequences for Higher-Dimensional Models}

6.1. Lattice Schrödinger operators with separable potentials. Let $d \geq 1$ be an integer and assume that for $1 \leq j \leq d$, we have bounded maps $V_{j}: \mathbb{Z} \rightarrow \mathbb{R}$. Consider the associated Schrödinger operators on $\ell^{2}(\mathbb{Z})$,

$$
\left[H_{j} \psi\right](n)=\psi(n+1)+\psi(n-1)+V_{j}(n) \psi(n) .
$$

Furthermore, we let $V: \mathbb{Z}^{d} \rightarrow \mathbb{R}$ be given by

$$
V(n)=V_{1}\left(n_{1}\right)+\cdots+V_{d}\left(n_{d}\right)
$$

where we express an element $n$ of $\mathbb{Z}^{d}$ as $n=\left(n_{1}, \ldots, n_{d}\right)$ with $n_{j} \in \mathbb{Z}$.

Finally, we introduce the Schrödinger operator on $\ell^{2}\left(\mathbb{Z}^{d}\right)$ with potential $V$, that is,

$$
[H \psi](n)=\left(\sum_{j=1}^{d} \psi\left(n+e_{j}\right)+\psi\left(n-e_{j}\right)\right)+V(n) \psi(n) .
$$

Here, $e_{j}$ denotes the element $n$ of $\mathbb{Z}^{d}$ that has $n_{j}=1$ and $n_{k}=0$ for $k \neq j$.

Potentials of the form (43) and Schrödinger operators of the form (44) are called separable. Let us first state some known results for separable Schrödinger operators.

Proposition 6.1. (a) The spectrum of $H$ is given by

$$
\sigma(H)=\sigma\left(H_{1}\right)+\cdots+\sigma\left(H_{d}\right)
$$

(b) Given $\psi_{1}, \ldots, \psi_{d} \in \ell^{2}(\mathbb{Z})$, denote by $\mu_{j}$ the spectral measure corresponding to $H_{j}$ and $\psi_{j}$. Furthermore, denote by $\mu$ the spectral measure corresponding to $H$ and the element $\psi$ of $\ell^{2}\left(\mathbb{Z}^{d}\right)$ given by $\psi(n)=\psi_{1}\left(n_{1}\right) \cdots \psi_{d}\left(n_{d}\right)$. Then,

$$
\mu=\mu_{1} * \cdots * \mu_{d} \text {. }
$$

Proof. Recall the definition and properties of tensor products of Hilbert spaces and operators on these spaces; see, for example, [RS, Sects. II.4 and VIII.10]. It follows from $\left[\mathrm{RS}\right.$, Theorem II.10] that there is a unique unitary map $U$ from $\ell^{2}(\mathbb{Z}) \otimes \cdots \otimes \ell^{2}(\mathbb{Z})$ ( $d$ factors) to $\ell^{2}\left(\mathbb{Z}^{d}\right)$ so that for $\psi_{j} \in \ell^{2}(\mathbb{Z})$, the elementary tensor $\psi_{1} \otimes \cdots \otimes \psi_{d}$ is mapped to the element $\psi$ of $\ell^{2}\left(\mathbb{Z}^{d}\right)$ given by $\psi(n)=\psi_{1}\left(n_{1}\right) \cdots \psi_{d}\left(n_{d}\right)$. With this unitary map $U$, we have

$$
U^{*} H U=\sum_{j=1}^{d} \mathrm{Id} \otimes \cdots \otimes \mathrm{Id} \otimes H_{j} \otimes \mathrm{Id} \otimes \cdots \otimes \mathrm{Id},
$$

with $H_{j}$ being the $j^{\text {th }}$ factor. Given this representation, part (a) now follows from [RS, Theorem VIII.33] (see also the example on [RS, p. 302]). Part (b) follows from the proof of [RS, Theorem VIII.33]; see also [BS] and [S]. 
6.2. A consequence of the Newhouse Gap Lemma. We have seen above that the spectrum of a product model is given by the sum of the individual spectra. If these individual spectra are Cantor sets and we want to show that their sum is not a Cantor set, the following consequence of the Gap Lemma is useful.

Lemma 6.2. Suppose $C, K \subset \mathbb{R}^{1}$ are Cantor sets with $\tau(C) \cdot \tau(K)>1$. Assume also that the size of the largest gap of $C$ is not greater than the diameter of $K$, and the size of the largest gap of $K$ is not greater than the diameter of $C$. Then the sum $C+K$ is a closed interval.

Proof. Denote $\min C=c_{1}, \max C=c_{2}, \min K=k_{1}, \max K=k_{2}$. Let us prove that

$$
C+K=\left[c_{1}+k_{1}, c_{2}+k_{2}\right] \text {. }
$$

The inclusion " $\subseteq$ " is obvious, so let us prove the inclusion " $\supseteq$." Take an arbitrary point $x \in\left[c_{1}+k_{1}, c_{2}+k_{2}\right]$. Then, $x \in C+K$ if and only if $0 \in C+K-x=C-(x-K)$. Therefore,

$$
x \in C+K \Leftrightarrow C \cap(x-K) \neq \emptyset .
$$

Since $\tau(C) \cdot \tau(x-K)=\tau(C) \cdot \tau(K)>1$, the Gap Lemma implies that a priori there are only four possibilities:

(1) the intervals $\left[c_{1}, c_{2}\right]$ and $\left[x-k_{2}, x-k_{1}\right]$ are disjoint;

(2) the set $C$ is contained in a finite gap of the set $(x-K)$;

(3) the set $(x-K)$ is contained in a finite gap of the set $C$;

(4) $C \cap(x-K) \neq \emptyset$.

But the case (1) contradicts the assumption $x \in\left[c_{1}+k_{1}, c_{2}+k_{2}\right]$, and the cases (2) and (3) are impossible due to our assumption on the sizes of gaps and diameters of $C$ and $K$. Therefore, we must have $C \cap(x-K) \neq \varnothing$ and hence $x \in C+K$.

6.3. The square Fibonacci Hamiltonian. Let us now discuss the (diagonal version of the) model studied by Even-Dar Mandel and Lifshitz, namely the operator

$$
\begin{aligned}
{\left[H_{V}^{(2)} \psi\right](m, n)=} & \psi(m+1, n)+\psi(m-1, n)+\psi(m, n+1)+\psi(m, n-1)+ \\
& +V\left(\chi_{[1-\alpha, 1)}(m \alpha \bmod 1)+\chi_{[1-\alpha, 1)}(n \alpha \bmod 1)\right) \psi(m, n)
\end{aligned}
$$

in $\ell^{2}\left(\mathbb{Z}^{2}\right)$. By Proposition 6.1, we have

$$
\sigma\left(H_{V}^{(2)}\right)=\Sigma_{V}+\Sigma_{V}
$$

Proof of Theorem 1.4. By (45), it suffices to show that $\Sigma_{V}+\Sigma_{V}$ is an interval for $V>0$ sufficiently small. By Theorem 1.2 , there is $V_{0}>0$ such that $\tau\left(\Sigma_{V}\right)>1$ for $V \in\left(0, V_{0}\right)$. For such $V$ 's, Lemma 6.2 applies with $C=K=\Sigma_{V}$ and yields

$$
\Sigma_{V}+\Sigma_{V}=\left[\min \Sigma_{V}+\min \Sigma_{V}, \max \Sigma_{V}+\max \Sigma_{V}\right]
$$

as desired.

Acknowledgement. We are grateful to Mark Embree for generating the plots shown in Figs. 4 and 5.

Open Access This article is distributed under the terms of the Creative Commons Attribution Noncommercial License which permits any noncommercial use, distribution, and reproduction in any medium, provided the original author(s) and source are credited. 


\section{Appendix A. The Off-Diagonal Fibonacci Hamiltonian}

The purpose of this appendix is to give complete proofs of the basic spectral properties of the off-diagonal Fibonacci Hamiltonian. This operator has been considered in many physics papers and is the basic building block for the higher-dimensional product models studied by Even-Dar Mandel and Lifshitz. The mathematics literature on the Fibonacci model provides an exhaustive study of the diagonal model, and it was always understood that "analogous results hold for the off-diagonal model." For the reader's convenience, we make these analogous results explicit here and hence provide in this paper a complete treatment of the spectrum of the Even-Dar Mandel-Lifshitz product model at weak coupling.

A.1. Model and results. Let $a, b$ be two positive real numbers and consider the Fibonacci substitution,

$$
S(a)=a b, \quad S(b)=a .
$$

This substitution rule extends to finite and one-sided infinite words by concatenation. For example, $S(a b a)=a b a a b$. Since $S(a)$ begins with $a$, one obtains a one-sided infinite sequence that is invariant under $S$ by iterating the substitution rule on $a$ and taking a limit. Indeed, we have

$$
S^{k}(a)=S^{k-1}(S(a))=S^{k-1}(a b)=S^{k-1}(a) S^{k-1}(b)=S^{k-1}(a) S^{k-2}(a) .
$$

In particular, $S^{k}(a)$ starts with $S^{k-1}(a)$, and hence there is a unique one-sided infinite sequence $u$, the so-called Fibonacci substitution sequence, that starts with $S^{k}(a)$ for every $k$. The hull $\Omega_{a, b}$ is then obtained by considering all two-sided infinite sequences that locally look like $u$,

$$
\Omega_{a, b}=\left\{\omega \in\{a, b\}^{\mathbb{Z}} \text { : every subword of } \omega \text { is a subword of } u\right\} .
$$

It is known that, conversely, every subword of $u$ is a subword of every $\omega \in \Omega_{a, b}$. In this sense, $u$ and all elements of the hull $\omega$ look exactly the same locally.

We wish to single out a special element of $\Omega_{a, b}$. Notice that $b a$ occurs in $u$ and that $S^{2}(a)=a b a$ begins with $a$ and $S^{2}(b)=a b$ ends with $b$. Thus, iterating $S^{2}$ on $b \mid a$, where | denotes the eventual origin, we obtain as a limit a two-sided infinite sequence which belongs to $\Omega_{a, b}$ and coincides with $u$ to the right of the origin. This element of $\Omega_{a, b}$ will be denoted by $\omega_{s}$.

Each $\omega \in \Omega_{a, b}$ generates a Jacobi matrix $H_{\omega}$ acting in $\ell^{2}(\mathbb{Z})$,

$$
\left(H_{\omega} \psi\right)_{n}=\omega_{n+1} \psi_{n+1}+\omega_{n} \psi_{n-1} \text {. }
$$

With respect to the standard orthonormal basis $\left\{\delta_{n}\right\}_{n \in \mathbb{Z}}$ of $\ell^{2}(\mathbb{Z})$, where $\delta_{n}$ is one at $n$ and vanishes otherwise, this operator has the matrix

$$
\left(\begin{array}{cccccc}
\ddots & \ddots & \ddots & & & \\
\ddots & 0 & \omega_{-1} & 0 & & \\
\ddots & \omega_{-1} & 0 & \omega_{0} & 0 & \\
& 0 & \omega_{0} & 0 & \omega_{1} & \ddots \\
& & 0 & \omega_{1} & 0 & \ddots \\
& & & \ddots & \ddots & \ddots
\end{array}\right)
$$

and it is clearly self-adjoint. 
This family of operators, $\left\{H_{\omega}\right\}_{\omega \in \Omega_{a, b}}$, is called the off-diagonal Fibonacci model. Of course, the structure of the Fibonacci sequence disappears when $a=b$. In this case, the hull consists of a single element, the constant two-sided infinite sequence taking the value $a=b$, and the spectrum and the spectral measures of the associated operator $H_{\omega}$ are well understood. For this reason, we will below always assume that $a \neq b$. Nevertheless, the limiting case, where we fix $a$, say, and let $b$ tend to $a$ is of definite interest.

Our first result concerns general properties of the spectrum of $H_{\omega}$. For $S \subset \mathbb{R}$, we denote by $\operatorname{dim}_{H} S$ the Hausdorff dimension of $S$ and by $\operatorname{dim}_{B} S$ the box counting dimension of $S$ (which is then implicitly claimed to exist).

Theorem A.1. Suppose $a, b>0$ and $a \neq b$. Then, there exists a compact set $\Sigma_{a, b} \subset \mathbb{R}$ such that $\sigma\left(H_{\omega}\right)=\Sigma_{a, b}$ for every $\omega \in \Omega_{a, b}$, and

(i) $\Sigma_{a, b}$ has zero Lebesgue measure.

(ii) The Hausdorff dimension $\operatorname{dim}_{H} \Sigma_{a, b}$ is an analytic function of $a$ and $b$.

(iii) $0<\operatorname{dim}_{H} \Sigma_{a, b}<1$.

More can be said about the spectrum when $a$ and $b$ are close to each other:

Theorem A.2. There exists $\varepsilon_{0}>0$ such that if $a, b>0, a \neq b$, and $\frac{a^{2}+b^{2}}{2 a b}<1+\varepsilon_{0}$ (in other words, if $a$ and $b$ are close enough), then

(iv) The spectrum $\Sigma_{a, b}$ is a Cantor set that depends continuously on $a$ and $b$ in the Hausdorff metric.

(v) For every small $\delta>0$ and every $E \in \Sigma_{a, b}$, we have

$$
\begin{aligned}
\operatorname{dim}_{H}\left((E-\delta, E+\delta) \cap \Sigma_{a, b}\right) & =\operatorname{dim}_{B}\left((E-\delta, E+\delta) \cap \Sigma_{a, b}\right) \\
& =\operatorname{dim}_{H} \Sigma_{a, b}=\operatorname{dim}_{B} \Sigma_{a, b} .
\end{aligned}
$$

(vi) Denote $\alpha=\operatorname{dim}_{H} \Sigma_{a, b}$, then the Hausdorff $\alpha$-measure of $\Sigma_{a, b}$ is positive and finite.

(vii) We have that

$$
\Sigma_{a, b}+\Sigma_{a, b}=\left[\min \Sigma_{a, b}+\min \Sigma_{a, b}, \max \Sigma_{a, b}+\max \Sigma_{a, b}\right]
$$

Given these results, and especially Theorem A.2.(vii), we can confirm rigorously the observation made by Even-Dar Mandel and Lifshitz in [EL06,EL07] that the square Fibonacci Hamiltonian (based on the off-diagonal one-dimensional model) has no gaps in its spectrum for sufficiently small coupling.

Next, we turn to the spectral type of $H_{\omega}$.

Theorem A.3. Suppose $a, b>0$ and $a \neq b$. Then, for every $\omega \in \Omega_{a, b}, H_{\omega}$ has purely singular continuous spectrum.

Throughout the rest of this appendix we will only consider $a, b>0$ with $a \neq b$. Theorems A.1 and A.2 are proved in Subsect. A. 2 and Theorem A.3 is proved in Subsect. A.3. 
A.2. The trace map and its relation to the spectrum. The spectral properties of $H_{\omega}$ are closely related to the behavior of the solutions to the difference equation

$$
\omega_{n+1} u_{n+1}+\omega_{n} u_{n-1}=E u_{n}
$$

Denote

$$
U_{n}=\left(\begin{array}{c}
u_{n} \\
\omega_{n} u_{n-1}
\end{array}\right)
$$

Then $u$ solves (47) (for every $n \in \mathbb{Z}$ ) if and only if $U$ solves

$$
U_{n}=T_{\omega}(n, E) U_{n-1},
$$

(for every $n \in \mathbb{Z}$ ), where

$$
T_{\omega}(n, E)=\frac{1}{\omega_{n}}\left(\begin{array}{cc}
E & -1 \\
\omega_{n}^{2} & 0
\end{array}\right)
$$

Note that $\operatorname{det} T_{\omega}(n, E)=1$. Iterating (48), we find

$$
U_{n}=M_{\omega}(n, E) U_{0},
$$

where

$$
M_{\omega}(n, E)=T_{\omega}(n, E) \times \cdots T_{\omega}(1, E) .
$$

With the Fibonacci numbers $\left\{F_{k}\right\}$, generated by $F_{0}=F_{1}=1, F_{k+1}=F_{k}+F_{k-1}$ for $k \geq 1$, we define

$$
x_{k}=x_{k}(E)=\frac{1}{2} \operatorname{Tr} M_{\omega_{s}}\left(F_{k}, E\right) .
$$

For example, we have

$$
\begin{aligned}
M_{\omega_{s}}\left(F_{1}, E\right) & =\frac{1}{a}\left(\begin{array}{cc}
E & -1 \\
a^{2} & 0
\end{array}\right), \\
M_{\omega_{s}}\left(F_{2}, E\right) & =\frac{1}{b}\left(\begin{array}{cc}
E & -1 \\
b^{2} & 0
\end{array}\right) \frac{1}{a}\left(\begin{array}{cc}
E & -1 \\
a^{2} & 0
\end{array}\right)=\frac{1}{a b}\left(\begin{array}{cc}
E^{2}-a^{2} & -E \\
E b^{2} & -b^{2}
\end{array}\right), \\
M_{\omega_{s}}\left(F_{3}, E\right) & =\frac{1}{a}\left(\begin{array}{cc}
E & -1 \\
a^{2} & 0
\end{array}\right) \frac{1}{b}\left(\begin{array}{cc}
E & -1 \\
b^{2} & 0
\end{array}\right) \frac{1}{a}\left(\begin{array}{cc}
E & -1 \\
a^{2} & 0
\end{array}\right) \\
& =\frac{1}{a^{2} b}\left(\begin{array}{cc}
E^{3}-E a^{2}-E b^{2} & -E^{2}+b^{2} \\
E^{2} a^{2}-a^{4} & -E a^{2}
\end{array}\right),
\end{aligned}
$$

and hence

$$
x_{1}=\frac{E}{2 a}, \quad x_{2}=\frac{E^{2}-a^{2}-b^{2}}{2 a b}, \quad x_{3}=\frac{E^{3}-2 E a^{2}-E b^{2}}{2 a^{2} b} .
$$


Lemma A.4. We have

$$
x_{k+1}=2 x_{k} x_{k-1}-x_{k-2}
$$

for $k \geq 2$. Moreover, the quantity

$$
I_{k}=x_{k+1}^{2}+x_{k}^{2}+x_{k-1}^{2}-2 x_{k+1} x_{k} x_{k-1}-1
$$

is independent of both $k$ and $E$ and it is given by

$$
I=\frac{\left(a^{2}+b^{2}\right)^{2}}{4 a^{2} b^{2}}-1 .
$$

Proof. Since $\omega_{s}$ restricted to $\{n \geq 1\}$ coincides with $u$ and the prefixes $s_{k}$ of $u$ of length $F_{k}$ obey $s_{k+1}=s_{k} s_{k-1}$ for $k \geq 2$ by construction (cf. (46)), the recursion (50) follows as in the diagonal case; compare [D00,D07a,S87]. This recursion in turn implies readily that (51) is $k$-independent.

In particular, the $x_{k}$ 's are again generated by the trace map

$$
T(x, y, z)=(2 x y-z, x, y)
$$

and the preserved quantity is again

$$
I(x, y, z)=x^{2}+y^{2}+z^{2}-2 x y z-1 .
$$

The only difference between the diagonal and the off-diagonal model can be found in the initial conditions. How are $x_{1}, x_{0}, x_{-1}$ obtained? Observe that the trace map is invertible and hence we can apply its inverse twice to the already defined quantity $\left(x_{3}, x_{2}, x_{1}\right)$. We have

$$
T^{-1}(x, y, z)=(y, z, 2 y z-x)
$$

and hence, using (49),

$$
\begin{aligned}
\left(x_{1}, x_{0}, x_{-1}\right) & =T^{-2}\left(x_{3}, x_{2}, x_{1}\right) \\
& =T^{-2}\left(\frac{E^{3}-2 E a^{2}-E b^{2}}{2 a^{2} b}, \frac{E^{2}-a^{2}-b^{2}}{2 a b}, \frac{E}{2 a}\right) \\
& =T^{-1}\left(\frac{E^{2}-a^{2}-b^{2}}{2 a b}, \frac{E}{2 a}, 2 \frac{\left(E^{2}-a^{2}-b^{2}\right) E}{4 a^{2} b}-\frac{E^{3}-2 E a^{2}-E b^{2}}{2 a^{2} b}\right) \\
& =T^{-1}\left(\frac{E^{2}-a^{2}-b^{2}}{2 a b}, \frac{E}{2 a}, \frac{E}{2 b}\right) \\
& =\left(\frac{E}{2 a}, \frac{E}{2 b}, 2 \frac{E^{2}}{4 a b}-\frac{E^{2}-a^{2}-b^{2}}{2 a b}\right) \\
& =\left(\frac{E}{2 a}, \frac{E}{2 b}, \frac{a^{2}+b^{2}}{2 a b}\right) .
\end{aligned}
$$

It follows that

$$
\begin{aligned}
I\left(x_{k+1}, x_{k}, x_{k-1}\right) & =I\left(x_{1}, x_{0}, x_{-1}\right) \\
& =\frac{E^{2}}{4 a^{2}}+\frac{E^{2}}{4 b^{2}}+\frac{\left(a^{2}+b^{2}\right)^{2}}{4 a^{2} b^{2}}-2 \frac{E^{2}\left(a^{2}+b^{2}\right)}{8 a^{2} b^{2}}-1 \\
& =\frac{\left(a^{2}+b^{2}\right)^{2}}{4 a^{2} b^{2}}-1
\end{aligned}
$$

for every $k \geq 0$. 
It is of crucial importance for the spectral analysis that, as in the diagonal case, the invariant is energy-independent and strictly positive when $a \neq b$ !

Lemma A.5. The spectrum of $H_{\omega}$ is independent of $\omega$ and may be denoted by $\Sigma_{a, b}$. With

$$
\sigma_{k}=\left\{E \in \mathbb{R}:\left|x_{k}\right| \leq 1\right\},
$$

we have

$$
\Sigma_{a, b}=\bigcap_{k \geq 1} \sigma_{k} \cup \sigma_{k+1}
$$

Moreover, for every $E \in \Sigma_{a, b}$ and $k \geq 2$,

$$
\left|x_{k}\right| \leq 1+\left(\frac{\left(a^{2}+b^{2}\right)^{2}}{4 a^{2} b^{2}}-1\right)^{1 / 2}
$$

and for $E \notin \Sigma_{a, b},\left|x_{k}\right|$ diverges super-exponentially.

Proof. It is well known that the hull $\Omega_{a, b}$ together with the standard shift transformation is minimal. In particular, every $\omega \in \Omega_{a, b}$ may be approximated pointwise by a sequence of shifts of any other $\tilde{\omega} \in \Omega_{a, b}$. The associated operators then converge strongly and we get $\sigma\left(H_{\omega}\right) \subseteq \sigma\left(H_{\tilde{\omega}}\right)$. Reversing the roles of $\omega$ and $\tilde{\omega}$, the first claim follows.

So let $\Sigma_{a, b}$ denote the common spectrum of the operators $H_{\omega}, \omega \in \Omega_{a, b}$. We have $\left\|H_{\omega}\right\| \leq \max \{2 a, 2 b\}$. Thus, $\Sigma_{a, b} \subseteq[-\max \{2 a, 2 b\}, \max \{2 a, 2 b\}]=: I_{a, b}$. For $E \in$ $I_{a, b}$, we have that at least one of $x_{1}, x_{0}$ belongs to [-1,1]. This observation allows us to use the exact same arguments Sütô used to prove (52) for the diagonal model in [S87].

The only point where care needs to be taken is the claim that $\sigma_{k}$ is the spectrum of the periodic Jacobi matrix obtained by repeating the values $\omega_{s}$ takes on $\left\{1 \leq n \leq F_{k}\right\}$ periodically on the off-diagonals. This, however, follows from the general theory of periodic Jacobi matrices, which relies on the diagonalization of the monodromy matrix (which is $M_{\omega_{s}}\left(F_{k}, E\right)$ in this case) in order to obtain Floquet solutions and in particular discriminate between those energies that permit exponentially growing solutions and those that do not. This distinction works just as well here, but one needs to use that the $\omega_{n}$ 's that enter in the $U_{n}$ 's are uniformly bounded away from zero and infinity.

Thus, after paying attention to this fact, we may now proceed along the lines of Sütô. Let us describe the main steps of the argument. Since at least one of $x_{1}, x_{0}$ belongs to $[-1,1]$, we have a result analogous to [S87, Lemma 2] with the same proof as given there. Namely, the sequence $\left\{x_{k}\right\}_{k \geq 0}$ is unbounded if and only if there exists $k$ such that $\left|x_{k}\right|>1$ and $\left|x_{k+1}\right|>1$. Moreover, we then have $\left|x_{k+l}\right|>c^{F_{l}}$ for some $c>1$ and all $l \geq 0$. This shows

$$
\sigma_{k} \cup \sigma_{k+1}=\bigcup_{l \geq 0} \sigma_{k+l}
$$

Using now the fact that the $F_{k}$ periodic Jacobi matrices with spectrum $\sigma_{k}$ converge strongly to $H_{\omega_{s}}$, we obtain

$$
\Sigma_{a, b} \subseteq \bigcap_{k \geq 1} \overline{\bigcup_{l \geq 0} \sigma_{k+l}}=\bigcap_{k \geq 1} \overline{\sigma_{k} \cup \sigma_{k+1}}=\bigcap_{k \geq 1} \sigma_{k} \cup \sigma_{k+1},
$$

since the spectra $\sigma_{k}$ and $\sigma_{k+1}$ are closed sets. Thus, we have one inclusion in (52). 
Next, suppose $E \in \bigcap_{k \geq 1} \sigma_{k} \cup \sigma_{k+1}$. If $k \geq 1$ is such that $\left|x_{k}\right|>1$, then $\left|x_{k-1}\right| \leq 1$ and $\left|x_{k+1}\right| \leq 1$. Since we have

$$
x_{k+1}^{2}+x_{k}^{2}+x_{k-1}^{2}-2 x_{k+1} x_{k} x_{k-1}-1=\frac{\left(a^{2}+b^{2}\right)^{2}}{4 a^{2} b^{2}}-1,
$$

this implies

$$
x_{k}=x_{k+1} x_{k-1} \pm\left(1-x_{k+1}^{2}-x_{k-1}^{2}+x_{k+1}^{2} x_{k-1}^{2}+\frac{\left(a^{2}+b^{2}\right)^{2}}{4 a^{2} b^{2}}-1\right)^{1 / 2},
$$

and hence

$$
\left|x_{k}\right| \leq\left|x_{k+1} x_{k-1}\right|+\left(\left(1-x_{k+1}^{2}\right)\left(1-x_{k-1}^{2}\right)+\left(\frac{\left(a^{2}+b^{2}\right)^{2}}{4 a^{2} b^{2}}-1\right)\right)^{1 / 2}
$$

which, using $\left|x_{k-1}\right| \leq 1$ and $\left|x_{k+1}\right| \leq 1$ again, implies the estimate (53) for $E \in$ $\bigcap_{k>1} \sigma_{k} \cup \sigma_{k+1}$. We will show in the next subsection that the boundedness of the sequence $\left\{x_{k}\right\}_{k \geq 0}$ implies that, for arbitrary $\omega \in \Omega_{a, b}$, no solution of the difference equation (47) is square-summable at $+\infty$. Consequently, such $E$ 's belong to $\Sigma_{a, b} .^{7}$ This shows the other inclusion in (52) and hence establishes it. Moreover, it follows that (53) holds for every $E \in \Sigma_{a, b}$.

Finally, from the representation (52) of $\Sigma_{a, b}$ and our observation above about unbounded sequences $\left\{x_{k}\right\}_{k \geq 0}$, we find that $\left|x_{k}\right|$ diverges super-exponentially for $E \notin$ $\Sigma_{a, b}$. This concludes the proof of the lemma.

Lemma A.6. For every $E \in \mathbb{R}$, there is $\gamma(E) \geq 0$ such that

$$
\lim _{n \rightarrow \infty} \frac{1}{n} \log \left\|M_{\omega}(n, E)\right\|=\gamma(E)
$$

uniformly in $\omega \in \Omega_{a, b}$.

Proof. This follows directly from the uniform subadditive ergodic theorem; compare [DL99b,Ho].

Lemma A.7. The set $\mathcal{Z}_{a, b}:=\{E \in \mathbb{R}: \gamma(E)=0\}$ has zero Lebesgue measure.

Proof. This is one of the central results of Kotani theory; see [Ko] and also [D07b]. Note that these papers only discuss the diagonal model. Kotani theory for Jacobi matrices is discussed in Carmona-Lacroix [CL] and the result needed can be deduced from what is presented there. For a recent reference that states a result sufficient for our purpose explicitly, see Remling [Re].

Lemma A.8. We have $\Sigma_{a, b}=\mathcal{Z}_{a, b}$.

7 This follows by a standard argument: If $E \notin \Sigma_{a, b}$, then $\left(H_{\omega}-E\right)^{-1}$ exists and hence $\left(H_{\omega}-E\right)^{-1} \delta_{0}$ is an $\ell^{2}(\mathbb{Z})$ vector that solves (47) away from the origin. Choosing its values for $n \geq 1$, say, and then using (47) to extend it to all of $\mathbb{Z}$, we obtain a solution that is square-summable at $+\infty$. 
Proof. The inclusion $\Sigma_{a, b} \supseteq \mathcal{Z}_{a, b}$ holds by general principles. For example, one can construct Weyl sequences by truncation when $\gamma(E)=0$. The inclusion $\Sigma_{a, b} \subseteq \mathcal{Z}_{a, b}$ can be proved in two ways. Either one uses the boundedness of $x_{k}$ for energies $E \in \Sigma_{a, b}$ to prove explicit polynomial upper bounds for $\left\|M_{\omega}(n, E)\right\|$ (as in [IT] for $\omega=\omega_{s}$ or in [DL99b] for general $\omega \in \Omega_{a, b}$ ), or one combines the proof of the absence of decaying solutions at $+\infty$ for $E \in \Sigma_{a, b}$ given in the next subsection with Osceledec's Theorem, which states that $\gamma(E)>0$ would imply the existence of an exponentially decaying solution at $+\infty$. Here we use one more time that $U_{n}$ is comparable in norm to $\left(u_{n}, u_{n-1}\right)^{T}$.

Proof of Theorems A.1 and A.2. The existence of the uniform spectrum $\Sigma_{a, b}$ was shown in Lemma A.6 and the fact that $\Sigma_{a, b}$ has zero Lebesgue measure follows from Lemmas A.7 and A.8. The set of bounded orbits of the restriction of the trace map $T: \mathbb{R}^{3} \rightarrow \mathbb{R}^{3}$ to the invariant surface $I(x, y, z)=C \equiv \frac{\left(a^{2}+b^{2}\right)^{2}}{4 a^{2} b^{2}}-1, C>0$, is hyperbolic; see [Can] (and also [DG09a] for $C$ sufficiently small and [Cas] for $C$ sufficiently large). Due to Lemma A.5, the points of the spectrum correspond to the points of the intersection of the line of the initial conditions

$$
\ell_{a, b} \equiv\left\{\left(\frac{E}{2 a}, \frac{E}{2 b}, \frac{a^{2}+b^{2}}{2 a b}\right): E \in \mathbb{R}\right\}
$$

with the stable manifolds of the hyperbolic set of bounded orbits. Properties (ii) and (iii) can be proved in exactly the same way as Theorem 6.5 in [Can]. The line $\ell_{a, b}$ intersects the stable lamination of the hyperbolic set transversally for sufficiently small $C>0$, as can be shown in the same way as for the diagonal Fibonacci Hamiltonian with a small coupling constant; see [DG09a]. Therefore the spectrum $\Sigma_{a, b}$ for close enough $a$ and $b$ is a dynamically defined Cantor set, and the properties (iv)-(vi) follow; see [DEGT,DG09a,Ma,MM,P,PT] and references therein. The statement (vii) follows as in the diagonal case since the thickness of $\Sigma_{a, b}$ tends to infinity as $\frac{a^{2}+b^{2}}{2 a b}$ approaches 1 .

Notice that a proof of the transversality of the line $\ell_{a, b}$ to the stable lamination of the hyperbolic set of bounded orbits for arbitrary $a \neq b$ would imply the properties (iv)-(vi) for these values of $a$ and $b$.

A.3. Singular continuous spectrum. In this subsection we prove Theorem A.3. Given the results from the previous subsection, we can follow the proofs from the diagonal case quite closely.

Proof of Theorem A.3. Since the absence of absolutely continuous spectrum follows from zero measure spectrum, we only need to show the absence of point spectrum. It was shown by Damanik and Lenz [DL99a] that, given any $\omega \in \Omega_{a, b}$ and $k \geq 1$, the restriction of $\omega$ to $\{n \geq 1\}$ begins with a square

$$
\omega_{1} \ldots \omega_{2 F_{k}} \ldots=\omega_{1} \ldots \omega_{F_{k}} \omega_{1} \ldots \omega_{F_{k}} \ldots
$$

such that $\omega_{1} \ldots \omega_{F_{k}}$ is a cyclic permutation of $S^{k}(a)$. By cyclic invariance of the trace, it follows that $\operatorname{Tr} M_{\omega}\left(F_{k}, E\right)=2 x_{k}(E)$ for every $E$.

The Cayley-Hamilton Theorem, applied to $M_{\omega}\left(F_{k}, E\right)$, says that

$$
M_{\omega}\left(F_{k}, E\right)^{2}-\left(\operatorname{Tr} M_{\omega}\left(F_{k}, E\right)\right) M_{\omega}\left(F_{k}, E\right)+I=0,
$$


which, by the observations above, translates to

$$
M_{\omega}\left(2 F_{k}, E\right)-2 x_{k} M_{\omega}\left(F_{k}, E\right)+I=0 .
$$

If $E \in \Sigma_{a, b}$ and $u$ is a solution of the difference equation (47), it therefore follows that

$$
U\left(2 F_{k}+1\right)-2 x_{k} U\left(F_{k}+1\right)+U(1)=0 .
$$

If $u$ does not vanish identically, this shows that $u_{n} \nrightarrow \rightarrow 0$ as $n \rightarrow \infty$ since the $x_{k}$ 's are bounded above and the $\omega_{n}$ 's are bounded below away from zero. In particular, if $E \in \Sigma_{a, b}$, then no non-trivial solution of (47) is square-summable at $+\infty$ and hence $E$ is not an eigenvalue. It follows that the point spectrum of $H_{\omega}$ is empty.

\section{References}

[As00] Astels, S.: Cantor sets and numbers with restricted partial quotients. Trans. Amer. Math. Soc. 352, 133-170 (2000)

[As01] Astels, S.: Sums of numbers with small partial quotients. ii. J. Number Theory 91, 187-205 (2001)

[As02] Astels, S.: Sums of numbers with small partial quotients. Proc. Amer. Math. Soc. 130, 637-642 (2002)

[AJ] Avila, A., Jitomirskaya, S.: The ten martini problem. Ann. of Math. 170, 303-342 (2009)

[AS] Avron, J., Simon, B.: Almost periodic schrödinger operators. ii. the integrated density of states. Duke Math. J. 50, 369-391 (1983)

[BGJ] Baake, M., Grimm, U., Joseph, D.: Trace maps, invariants, and some of their applications. Internat. J. Mod. Phys. B 7, 1527-1550 (1993)

[BR] Baake, M., Roberts, J.: The dynamics of trace maps. In: Hamiltonian Mechanics (Toruń, 1993), NATO Adv. Sci. Inst. Ser. B Phys. 331, New York: Plenum, 1994, pp. 275-285

[B] Bellissard, J.: Spectral properties of Schrödinger's operator with a Thue-Morse potential. In: Number Theory and Physics (Les Houches, 1989), Springer Proc. Phys. 47, Berlin: Springer, 1990, pp. $140-150$

[BBG91] Bellissard, J., Bovier, A., Ghez, J.-M.: Spectral properties of a tight binding hamiltonian with period doubling potential. Commun. Math. Phys. 135, 379-399 (1991)

[BBG92] Bellissard, J., Bovier, A., Ghez, J.-M.: Gap labelling theorems for one-dimensional discrete schrödinger operators. Rev. Math. Phys. 4, 1-37 (1992)

[BS] Bellissard, J., Schulz-Baldes, H.: Subdiffusive quantum transport for $3 \mathrm{~d}$ hamiltonians with absolutely continuous spectra. J. Stat. Phys. 99, 587-594 (2000)

[BG] Bovier, A., Ghez, J.-M.: Remarks on the spectral properties of tight-binding and kronig-penney models with substitution sequences. J. Phys. A 28, 2313-2324 (1995)

[Can] Cantat, S.: Bers and hénon, painlevé and schrödinger. Duke Math. J. 149, 411-460 (2009)

[CL] Carmona, R., Lacroix, J.: Spectral Theory of Random Schrödinger Operators. Boston, MA: Birkhäuser, 1990

[Cas] Casdagli, M.: Symbolic dynamics for the renormalization map of a quasiperiodic schrödinger equation. Commun. Math. Phys. 107, 295-318 (1986)

[Cus] Cusick, T.: On M. Hall's continued fraction theorem. Proc. Amer. Math. Soc. 38, 253-254 (1973)

[CFKS] Cycon, H., Froese, R., Kirsch, W., Simon, B.: Schrödinger Operators with Application to Quantum Mechanics and Global Geometry. Texts and Monographs in Physics, Berlin: Springer-Verlag, 1987

[D00] Damanik, D.: Gordon-type arguments in the spectral theory of one-dimensional quasicrystals. In: Directions in Mathematical Quasicrystals. CRM Monogr. Ser. 13, Providence, RI: Amer. Math. Soc., 2000, pp. 277-305

[D07a] Damanik, D.: Strictly ergodic subshifts and associated operators. In: Spectral Theory and Mathematical Physics: a Festschrift in Honor of Barry Simon's 60th Birthday. Proc. Sympos. Pure Math. 76, Part 2, Providence, RI: Amer. Math. Soc., 2007, pp. 505-538

[D07b] Damanik, D.: Lyapunov exponents and spectral analysis of ergodic Schrödinger operators: a survey of Kotani theory and its applications. In: Spectral Theory and Mathematical Physics: a Festschrift in Honor of Barry Simon's 60th Birthday, Proc. Sympos. Pure Math. 76, Part 2, Providence, RI: Amer. Math. Soc., 2007, pp. 539-563

[DEGT] Damanik, D., Embree, M., Gorodetski, A., Tcheremchantsev, S.: The fractal dimension of the spectrum of the fibonacci hamiltonian. Commun. Math. Phys. 280, 499-516 (2008) 
[DG09a] Damanik, D., Gorodetski, A.: Hyperbolicity of the trace map for the weakly coupled fibonacci hamiltonian. Nonlinearity 22, 123-143 (2009)

[DG09b] Damanik, D., Gorodetski, A.: The spectrum of the weakly coupled fibonacci hamiltonian. Electronic Research Announcements in Mathematical Sciences 16, 23-29 (2009)

[DKL] Damanik, D., Killip, R., Lenz, D.: Uniform spectral properties of one-dimensional quasicrystals. iii. $\alpha$-continuity. Commun. Math. Phys. 212, 191-204 (2000)

[DL99a] Damanik, D., Lenz, D.: Uniform spectral properties of one-dimensional quasicrystals, i. absence of eigenvalues. Commun. Math. Phys. 207, 687-696 (1999)

[DL99b] Damanik, D., Lenz, D.: Uniform spectral properties of one-dimensional quasicrystals. ii. the lyapunov exponent. Lett. Math. Phys. 50, 245-257 (1999)

[DST] Damanik, D., Sütő, A., Tcheremchantsev, S.: Power-law bounds on transfer matrices and quantum dynamics in one dimension ii. J. Funct. Anal. 216, 362-387 (2004)

[DT03] Damanik, D., Tcheremchantsev, S.: Power-law bounds on transfer matrices and quantum dynamics in one dimension. Commun. Math. Phys. 236, 513-534 (2003)

[DT07] Damanik, D., Tcheremchantsev, S.: Upper bounds in quantum dynamics. J. Amer. Math. Soc. 20, 799-827 (2007)

[DT08] Damanik, D., Tcheremchantsev, S.: Quantum dynamics via complex analysis methods: general upper bounds without time-averaging and tight lower bounds for the strongly coupled fibonacci hamiltonian. J. Funct. Anal. 255, 2872-2887 (2008)

[EL06] Even-Dar Mandel, S., Lifshitz, R.: Electronic energy spectra and wave functions on the square fibonacci tiling. Phil. Mag. 86, 759-764 (2006)

[EL07] Even-Dar Mandel, S., Lifshitz, R.: Electronic energy spectra of square and cubic fibonacci quasicrystals. Phil. Mag. 88, 2261-2273 (2008)

[EL08] Even-Dar Mandel, S., Lifshitz, R.: Bloch-like electronic wave functions in two-dimensional quasicrystals. http://arXiv.org/abs/0808.3659IIVL[cond-mat.mtrl.sci], 2008

[Ha] Hall, M.: On the sum and product of continued fractions. Ann. of Math. 48, 966-993 (1947)

[Hl] Hlavka, J.: Results on sums of continued fractions. Trans. Amer. Math. Soc. 211, 123-134 (1975)

[HPS] Hirsch, M., Pugh, C., Shub, M.: Invariant Manifolds. Lecture Notes in Mathematics 583, BerlinNew York: Springer-Verlag, 1977

[Ho] Hof, A.: Some remarks on discrete aperiodic schrödinger operators. J. Stat. Phys. 72, 1353-1374 (1993)

[HCB] Hoggatt, V., Cox, N., Bicknell, M.: A primer for the fibonacci numbers. xii. Fibonacci Quart. 11, 317-331 (1973)

[HM] Humphries, S., Manning, A.: Curves of fixed points of trace maps. Ergod. Th. \& Dynam. Sys. 27, 1167-1198 (2007)

[IT] Iochum, B., Testard, D.: Power law growth for the resistance in the fibonacci model. J. Stat. Phys. 65, 715-723 (1991)

[Ka] Kadanoff, L.P.: Analysis of cycles for a volume preserving map, unpublished manuscript

[KKT] Kohmoto, M., Kadanoff, L.P., Tang, C.: Localization problem in one dimension: mapping and escape. Phys. Rev. Lett. 50, 1870-1872 (1983)

[Ko] Kotani, S.: Jacobi matrices with random potentials taking finitely many values. Rev. Math. Phys. 1, 129-133 (1989)

[La] Last, Y.: Quantum dynamics and decompositions of singular continuous spectra. J. Funct. Anal. 142, 406-445 (1996)

[LS] Last, Y., Simon, B.: Fine structure of the zeros of orthogonal polynomials. iv. a priori bounds and clock behavior. Comm. Pure Appl. Math. 61, 486-538 (2008)

[Le] Lekkerkerker, C.: Representation of natural numbers as a sum of fibonacci numbers. Simon Stevin 29, 190-195 (1952)

[LW] Liu, Q.-H., Wen, Z.-Y.: Hausdorff dimension of spectrum of one-dimensional schrödinger operator with sturmian potentials. Pot. Anal. 20, 33-59 (2004)

[Ma] Mané, R.: The hausdorff dimension of horseshoes of diffeomorphisms of surfaces. Bol. Soc. Brasil. Mat. (N.S.) 20, 1-24 (1990)

[Me] de Melo, W.: Structural stability of diffeomorphisms on two-manifolds. Invent. Math. 21, 233-246 (1973)

[MM] Manning, A., McCluskey, H.: Hausdorff dimension for horseshoes. Erg. Theory Dynam. Sys. 3, 251-261 (1983)

[N79] Newhouse, S.: The abundance of wild hyperbolic sets and nonsmooth stable sets for diffeomorphisms. Inst. Hautes Études Sci. Publ. Math. 50, 101-151 (1979)

[N70] Newhouse, S.: Nondensity of axiom A(a) on $S^{2}$. Global Analysis (Proc. Sympos. Pure Math., Vol. XIV, Berkeley, Calif., 1968), Providence, RI: Amer. Math. Soc., 1970, pp. 191-202

[OK] Ostlund, S., Kim, S.-H.: Renormalization of quasiperiodic mappings. Physica Scripta T9, 193-198 (1985) 
[PT] Palis, J., Takens, F.: Hyperbolicity and Sensitive Chaotic Dynamics at Homoclinic Bifurcations. Cambridge: Cambridge University Press, 1993

[P] Pesin, Ya.: Dimension Theory in Dynamical Systems. Chicago Lectures in Mathematics Series, Chicago, IL: Univ. Chicago Press, 1997

[PSW] Pugh, C., Shub, M., Wilkinson, A.: Hölder foliations. Duke Math. J. 86, 517-546 (1997)

[Ra] Raymond, L.: A constructive gap labelling for the discrete Schrödinger operator on a quasiperiodic chain. Preprint, 1997

[RS] Reed, M., Simon, B.: Methods of Modern Mathematical Physics. I. Functional Analysis, $2^{\text {nd }}$ edition, New York: Academic Press, 1980

[Re] Remling, C.: The absolutely continuous spectrum of Jacobi matrices. Preprint, 2007

[Ro] Roberts, J.: Escaping orbits in trace maps. Phys. A 228, 295-325 (1996)

[S] Simon, B.: Operators with singular continuous spectrum. vii. examples with borderline time decay. Commun. Math. Phys. 176, 713-722 (1996)

[Si89] Sire, C.: Electronic spectrum of a 2d quasi-crystal related to the octagonal quasi-periodic tiling. Europhys. Lett. 10, 483-488 (1989)

[SM89] Sire, C., Mosseri, R.: Spectrum of 1d quasicrystals near the periodic chain. J. Phys. France 50, 34473461 (1989)

[SM90] Sire, C., Mosseri, R.: Excitation spectrum, extended states, gap closing: some exact results for codimension one quasicrystals. J. Phys. France 51, 1569-1583 (1990)

[SMS] Sire, C., Mosseri, R., Sadoc, J.-F.: Geometric study of a 2d tiling related to the octagonal quasiperiodic tiling. J. Phys. France 55, 3463-3476 (1989)

[S87] Sütő, A.: The spectrum of a quasiperiodic schrödinger operator. Commun. Math. Phys. 111, 409415 (1987)

[S89] Sütô, A.: Singular continuous spectrum on a cantor set of zero lebesgue measure for the fibonacci hamiltonian. J. Stat. Phys. 56, 525-531 (1989)

[S95] Sütő, A.: Schrödinger difference equation with deterministic ergodic potentials. In: Beyond Quasicrystals (Les Houches, 1994), Berlin: Springer, 1995, pp. 481-549

[T] Takens, F.: Limit capacity and Hausdorff dimension of dynamically defined Cantor sets, Dynamical Systems, Lecture Notes in Mathematics 1331, Berlin: Springer, 1988, pp. 196-212

[Z] Zeckendorf, E.: A generalized fibonacci numeration. Fibonacci Quart. 10, 365-372 (1972)

Communicated by G. Gallavotti 